\title{
The role of protein phosphorylation in regulation of carbon catabolite repression in Bacillus subtilis
}

\author{
PhD Thesis
}

in partial fulfilment of the requirements for the degree "Doctor of Philosophy (PhD)" in the Molecular Biology Program at the Georg August University Göttingen,

Faculty of Biology

submitted by

Kalpana Singh

born in

Haryana, India 
I hereby declare that the $\mathrm{PhD}$ thesis entitled, "The role of protein phosphorylation in regulation of carbon catabolite repression in Bacillus subtilis" has been written independently and with no other sources and aids than quoted.

Kalpana Singh 


\section{Acknowledgement}

This thesis has been the culmination of the research work done in the Department of General Microbiology, University of Göttingen, under the supervision of Prof. Jörg Stülke and Dr. Boris Görke.

To begin with, I am thankful to Prof. Jörg Stülke for giving me the opportunity to carry out my dissertation in his department and for his constant support and encouragement.

I wish to express my sincerest gratitude to Dr. Boris Görke for his supervision, advice, and guidance. He gave constant encouragement and support in various ways. His scientific acumen has made him as a continous source of ideas which inspired and enriched my growth as a student, a researcher and a scientist want to be. I am indebted to him, for his faith in me and providing the right direction whenever I needed it the most.

I thank my doctoral committee members, Prof. Dr. Wolfgang Liebl and PD Dr. Markus Wahl for the "outside" perspective to my work and their time.

I am thankful to the financial support by the Lichtenberg fellowship, and the "Bridging funds" by GGNB.

I am grateful to Prof. Chester W. Price, Dr. David Rudner, Dr. Isabelle MartinVerstraete, Dr. Peter J. Lewis, Dr. Simone Séror, Dr. Thorsten Mascher, and Prof. Wolfgang Schumann for the generous gifts of plasmids and strains.

My heartfelt thanks to Sabine Lentes for her tremendous help and the much needed assistance and support. She made it possible for me to achieve the maximum in the shortest time span. I am thankful to her for the time and efforts she spent on carrying out the tedious Lac-Z measurements.

I am thankful to all the members of this department who helped me in one way or the other to carry out my work successfully. Especially Christina, her meticulous work habits and her comforting presence made the lab a pleasure to work in. I shall forever cherish and value her ideas and useful tips. I thank Fabian and Sven for answering my endless questions when I started my work in this Lab. Also, I thank Sebastian and Claudine for being interesting peers.

I wish to further extend my thanks to other past and present members of my workgroup. I thank Birte, who pitched in with interesting chit chat over coffee, for being a great friend and a mental support in the lab. Antje, Björn, Denise, Falk, Maria and Ricarda for providing a fun filled lab environment, constant help with personal and lab matters. Thanks to Birte and Falk again for critically reading some parts of my thesis. 
I thank Nicole, Ricarda and Maria for providing me an opportunity to supervise them. It was a learning process for me as well, while I was sharing my knowledge with them.

I am grateful to all my friends in Göttingen, who made my stay here a memorable one. To Bhavna as it has been a pleasure having her as a friend. I can not thank her enough for all the mental support she provided me while I was facing the most stressful times. She kept me going and I am extremely thankful for that. I am indebted to Oli, who continued to be a great friend while he was in the lab and then out of it. I surely know much more about "processors", "samplers" and "bikes" now.

I am thankful to Devan for being my home away from home in Göttingen. He has been an amazing friend and support and a constant source of joy for me. Thanks for that!

I would like to thank all the people whose names I did not include here, but they provided me with the necessary help and made it possible for me to write this thesis.

Finally, my parents and my siblings deserve special mention here. My deep and lasting gratitude to my mama and papa for their unconditional love, faith, and support. They have been a constant source of inspiration and this work is especially dedicated to them. To Alka and Sandeep for cheering me up whenever I felt low and being the most loving siblings. 


\section{Table of Contents}

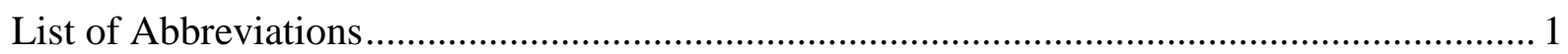

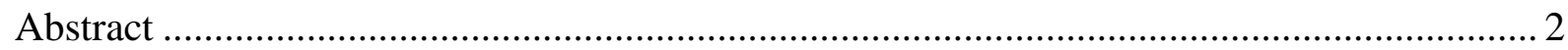

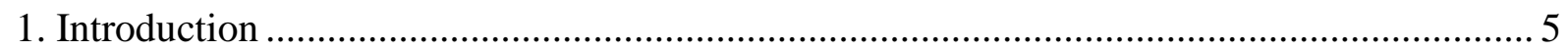

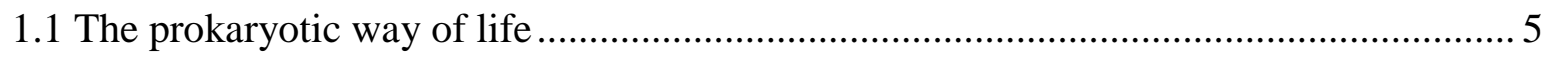

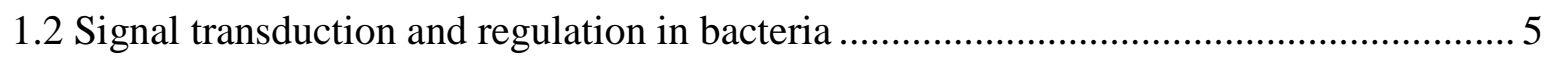

1.2.1 Modes of phosphorylation dependent signal transduction in Bacteria .................... 6

1.3 Sugar utilization and metabolism in $B$. subtilis .................................................... 8

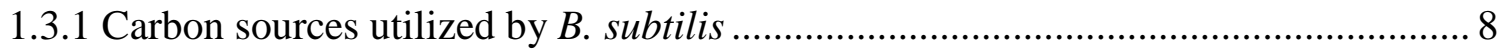

1.3.2 The PTS: an economical way of sugar uptake ..................................................

1.3.3 General pathways for the catabolism of carbohydrates........................11

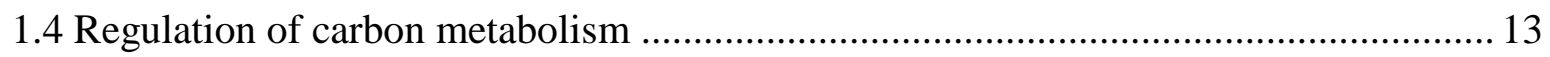

1.4.1 The regulatory role of PTS in CCR: linking sugar uptake and metabolism. .......... 14

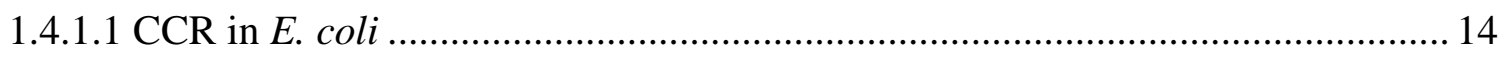

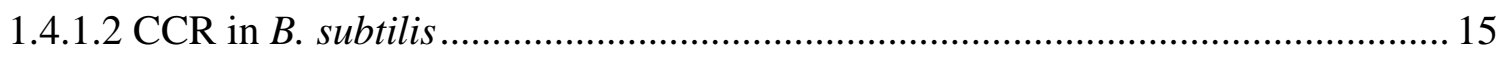

1.4.2 Global mechanism of carbon catabolite repression in B. subtilis .......................... 17

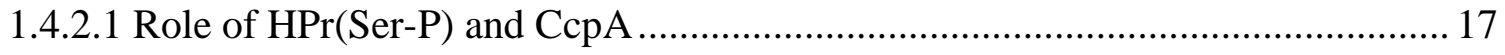

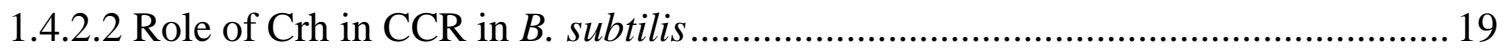

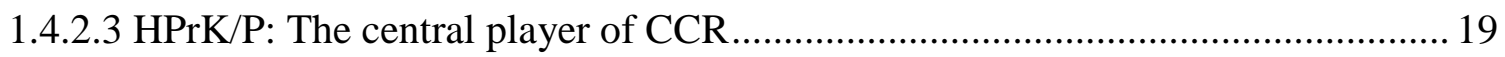

1.4.2.4 Interplay of protein factors (HPr, Crh, HPrK/P and CcpA) and small molecule

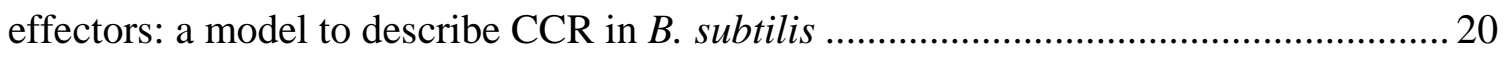

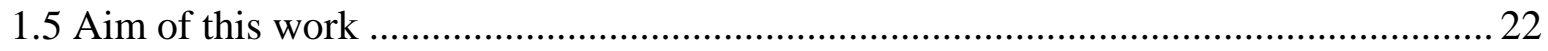

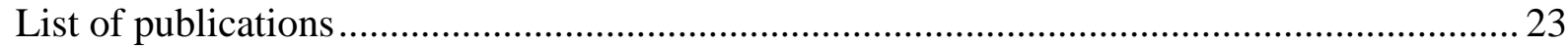

2. Control of the Phosphorylation State of the HPr Protein of the Phosphotransferase System in Bacillus subtilis: implication of the Protein Phosphatase PrpC

3. Carbon catabolite repression in Bacillus subtilis: A quantitative analysis of repression exerted by different carbon sources

4. Analysis of the hprK operon in Bacillus subtilis: Evidence for a role of a gene encoded downstream of $h p r K$ in carbon catabolite repression....................................................... 57

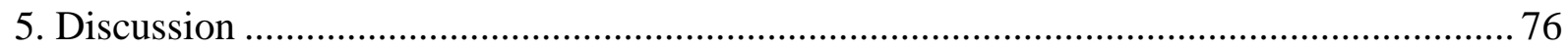

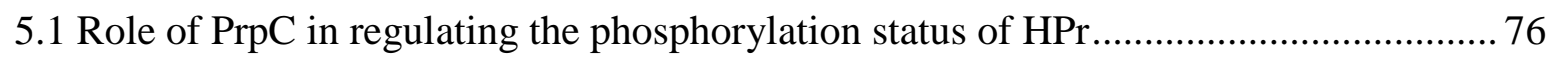

5.2 Hierarchical nature of catabolite repression exerted by various carbon sources ........... 79 
5.3 HPr(Ser-P) levels in the cell govern the strength of catabolite repression by various carbon sources 81

5.4 Modulation of the phosphorylation state of HPr by the nature of the carbon source..... 84

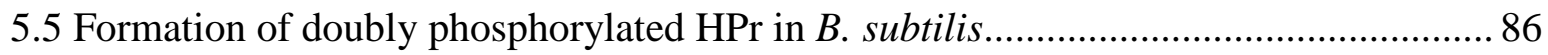

5.6 Activity of HPrK/P determines CCR in B. subtilis..............................90

Summary and Conclusions................................................93

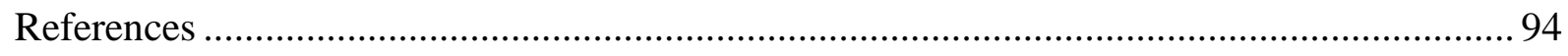

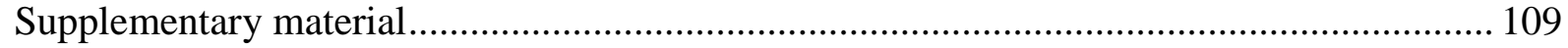

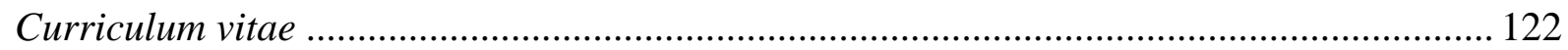




\section{List of Abbreviations}

\begin{tabular}{|c|c|}
\hline $\mathrm{ABC}$ & ATP binding cassette \\
\hline ATP & adenosine triphosphate \\
\hline $\mathrm{bp}$ & base pairs \\
\hline CCR & carbon catabolite repression \\
\hline DNA & deoxyribonucleic acid \\
\hline DTT & Dithiothreitol \\
\hline ermC & Erythromycin \\
\hline E I & enzyme I \\
\hline EII & enzyme II \\
\hline FBP & fructose 1,6-bisphosphate \\
\hline glu & Glucose \\
\hline G6P & glucose-6-phosphate \\
\hline His & Histidine \\
\hline IPTG & Isopropyl- $\beta$-D-thiogalactopyranoside \\
\hline $\mathrm{KDa}$ & Kilodaltons \\
\hline LB & Luria Bertani \\
\hline LFH & long flanking homology \\
\hline MIN & Minutes \\
\hline $\mathrm{mM}$ & Millimolar \\
\hline MW & molecular weight \\
\hline NTA & nitrilotriacetic acid \\
\hline OD & optical density \\
\hline ONPG & 2-nitrophenyl- $\beta$-D-galactosidase \\
\hline PAGE & polyacrylamide gel electrophoresis \\
\hline PCR & polymerase chain reaction \\
\hline PEP & Phosphoenolpyruvate \\
\hline $\mathrm{pH}$ & power of hydrogen \\
\hline PNPP & p-nitro phenyl phosphate \\
\hline PNPX & p-nitro phenyl xyloside \\
\hline PP2C & Protein phosphatase $2 \mathrm{C}$ \\
\hline PPP & pentose phosphate pathway \\
\hline PPP & protein phosphatase $\mathrm{P}$ \\
\hline p.s.i & per square inch \\
\hline PTS & phosphotransferase system \\
\hline PVDF & polyvinylidene difluoride membrane \\
\hline $\mathrm{rpm}$ & rotation per minute \\
\hline Ser & Serine \\
\hline Spc & Spectinomycin \\
\hline RNA & ribonucleic acid \\
\hline TCA & tricarboxylic acid \\
\hline Tris & Tris(hydroxymethyl)aminomethane \\
\hline $\mathrm{Wt} /$ vol & weight per volume \\
\hline WT & Wild type \\
\hline
\end{tabular}




\section{Abstract}

Bacteria have developed sophisticated mechanisms to adapt to a variety of environmental conditions, including the availability and supply of different carbon sources. If provided with a mixture of nutrients, many bacteria can selectively utilize the carbon source that allows the most rapid growth. Simultaneously, the cells repress the functions involved in the utilization of secondary carbon sources. The underlying phenomenon is called carbon catabolite repression (CCR). CCR is a paradigm of signal transduction and is achieved by different molecular mechanisms in Gram-negative and Gram-positive bacteria. In Bacillus subtilis, the transcriptional regulator $\mathrm{CcpA}$ is the master regulator of the global CCR. In the presence of a preferred carbon source, CcpA is activated and binds to its DNA operator sites, the catabolite responsive elements (cre). This leads to the repression of numerous genes involved in metabolism and utilization of secondary carbon sources. The DNA binding activity of CcpA is stimulated by interaction with its phosphorylated cofactors, $\operatorname{HPr}(\operatorname{Ser}-\mathrm{P})$ and $\mathrm{Crh}(\mathrm{Ser}-\mathrm{P})$. HPr and Crh are phosphorylated by the ATP dependent bifunctional enzyme, the HPrK/P at Ser-46 residue. HPrK/P is an allosteric enzyme and possesses both kinase and phosphorylase activity, thereby catalysing the (de)phosphorylation of HPr and Crh. The antagonistic activities of HPrK/P are triggered by metabolites such as fructose 1,6-bisphoaphate (FBP), ATP and inorganic phosphate $\left(\mathrm{P}_{\mathrm{i}}\right)$. HPr is also a part of the phosphoenol pyruvate dependent phosphotransferase system (PEP:PTS). The PTS is involved in carbohydrate uptake in many bacteria. When participating in sugar transport, HPr is phosphorylated at histidine-15 (His-P)

residue. The two phosphorylation events (Ser-P and His-P) are considered to be mutually exclusive and only $\operatorname{HPr}(\operatorname{Ser}-\mathrm{P})$ can participate in CCR. According to the present model of CCR in B. subtilis, the metabolism of a preferred carbon source increases the intracellular level of FBP. A high FBP concentration stimulates the HPrK/P kinase activity, leading to high amounts of intracellular $\mathrm{HPr}(\mathrm{Ser}-\mathrm{P})$, which causes CCR.

Glucose is the most preferred carbon source for many organisms and the current model of CCR in B. subtilis is based on studies, which focussed on glucose as the repressing carbon source. In this study, CCR was studied in the presence of various carbon sources, which are catabolised and taken up by different metabolic pathways. The different carbon sources formed a hierarchy in their ability to cause CCR. As a reporter system for CCR expression of the $\beta$-xylosidase (XynB) was analysed. Most of the strong repressing carbon sources were found to be PTS substrates. It could be demonstrated that repression by each 
carbon source is mediated via the CcpA dependent pathway, except for sorbitol. This highlights the existence of a CcpA independent mechanism of CCR of $x y n B$ expression.

For the CcpA dependent pathway of CCR, HPr was demonstrated to be the relevant corepressor in vivo. A strain lacking the second cofactor of CcpA, Crh exhibited similar repression of the reporter system as the wild type strain. Western blot analysis revealed that the amounts of HPr, HPrK/P and CcpA do not vary with the carbon source. This ruled out the possibility that the differential CCR exerted by the different carbon sources is caused by different intracellular amounts of these proteins. Therefore, the phosphorylation state of HPr in vivo was analysed. The presence of strong repressing carbon sources generated high intracellular $\operatorname{HPr}(\mathrm{Ser}-\mathrm{P})$ levels as compared to the weaker repressing carbon sources. Moreover, weaker repressing carbon sources allowed formation of large amounts of HPr(HisP). Thus, it could be well established that the different repressing potential of various carbon sources are derived from the ability to generate differential intracellular levels of $\mathrm{HPr}(\mathrm{Ser}-\mathrm{P})$.

I then addressed the question, how B. subtilis cells may judge the quality of a given carbon source, in terms of being a stronger or weaker repressing sugar. Since PTS carbon sources generally exerted a strong CCR, I analysed if an active PTS may affect the intracellular generation of $\operatorname{HPr}(\operatorname{Ser}-\mathrm{P})$. Preventing the phosphorylation at histidine residue of HPr, resulted in a complete relief from CCR by PTS substrates. This was expected because these substrates can not be taken up in this mutant. However, the repression by non-PTS substrates did not change as compared to a wild type strain. This interesting observation clearly indicated that even if HPr does not participate in PTS, its participation in CCR can not be increased. Hence, there is no competition between His- and Ser- phosphorylated froms of HPr. Thus, differences in $\operatorname{HPr}(\operatorname{Ser}-\mathrm{P})$ amounts must originate from the differential kinase activity of HPrK/P. Since the activity of the HPr kinase is believed to be triggered by FBP, we determined the intracellular FBP concentrations in cells grown on the different carbon sources. It turned out that on most sugars the intracellular FBP level is high enough to achieve full activation of the HPrK/P kinase activity. Thus, in contrast to the existing model, there must be further factors or metabolites, which affect the HPrK/P activity in vivo.

A hint regarding this possible additional factor, which may modulate the kinase activity of HPrK/P could also be gathered in this work. The relieved repression in a strain carrying a $\triangle h p r K$ polar mutation could not be restored by the ectopic expression of $h p r K$. This suggested a possible role of the genes downstream of $h p r K$ in CCR.

$\mathrm{HPr}(\operatorname{Ser}-\mathrm{P})$ is also known to be a target of the Ser/Thr phosphatase, PrpC in Mycoplasma pneumoniae. Thus, the possible role of this phosphatase in CCR was also 
analysed in $B$. subtilis. It could be established that $B$. subtilis PrpC can indeed dephosphorylate $\mathrm{HPr}(\mathrm{Ser}-\mathrm{P})$ in vitro. In vivo, a $\operatorname{prpC}$ mutation had a minor effect on the phosphorylation state of HPr and none on CCR.

In summary, the global CCR mechanism in B. subtilis is governed solely by HPrK/P activity. Depending on the available carbon source, a concerted action of known and unknown factors/metabolites leads to the modulation of HPrK/P activity, which ultimately determines the strength of CCR. 


\section{Introduction}

\subsection{The prokaryotic way of life}

For prokaryotes, adaptation is the key to survival. They rarely live in a constant environment and face challenges like changes in the physical and chemical composition of their habitats, limited nutrient supply etc. Moreover, in their natural habitat, bacteria have to compete with other microorganisms for the available resources.

Bacillus subtilis is a low $\mathrm{G}+\mathrm{C}$ content, heterotrophic, Gram-positive soil bacterium and has developed sophisticated mechanisms to thrive well in the rough conditions of its natural habitat. This is achieved primarily by the combination of an economical use of the limited nutrient sources and intricate gene/cell regulation mechanisms (Stülke and Hillen, 2000).

Likewise, the ability to sense and migrate towards preferred carbon and nitrogen sources allows the bacterium to make use of new resources and to escape from unfavourable conditions. Upon conditions of prolonged stress and nutrient deprivation, B. subtilis is able to initiate the process of sporulation. During sporulation, dormant, heat and stress resistant endospores are formed that allow B. subtilis to survive unfavourable conditions. The process of spore formation made B. subtilis a model organism for the study of this simple developmental process (Errington, 1993).

\subsection{Signal transduction and regulation in bacteria}

Signal transduction in any organism serves the purpose of mediating response to a chemical or physical signal from its environment. The resulting intracellular effects of this signal sensing can be a change in gene expression, alterations in metabolism, the continued proliferation and death of the cell or the stimulation or suppression of locomotion. Protein modification is one of the principal mechanisms for the regulation of cellular functions in response to extracellular stimuli in both eukaryotes and prokaryotes. In this respect, protein phosphorylation and dephosphorylation provides the cell with signals that reflect rapid changes in the cell physiology. The prominence of phosphorylation derives from various attributes. Primarily, the high charge density, the ability to form strong salt bridges and the propensity to form multiple hydrogen bonds renders phosphate a potent agent for altering the chemical structure of a protein (Johnson and Barford, 1993). In addition, phosphorylation events are reversible and thus, unlike other covalent modifications, proteins can be readily 
converted back to their native state in vivo. Thus, depending on the stimulus perceived, a protein can exist in physically and functionally distinguishable states.

A number of amino acid residues can accept phosphate. Commonly phosphorylated residues include histidine, tyrosine, serine, threonine and aspartate. Chemical properties and the intrinsic free energy of phosphorylation of the amino acids as compared to the same residue when they are part of a protein, also determines the cellular function of different types of phosphorylation. For instance, the free energy of hydrolysis of phosphoserines within proteins is higher than that of free phosphoserine, thus this mode of phosphorylation is usually involved in induction of energetically unfavourable conformational changes (Sprang et al., 1988). On the other hand, the free energy of phosphohistidines within proteins is similar to that of free phosphohistidines, suggesting that this phosphorylation is independent of the protein of which it is a part (Stock et al., 1990).

A number of cellular processes in prokaryotes including control of metabolism, carbohydrate transport, gene transcription, cellular differentiation, infective mechanisms of pathogens, osmoregulation, chemotaxis and phototaxis are subjected to phosphorylation dependent regulation (Alex and Simon, 1994; Cozzone, 1993; Mann, 1994; Stock et al., 1992).

\subsubsection{Modes of phosphorylation dependent signal transduction in Bacteria}

In bacteria two main modes of signal transduction prevail. Firstly, the extracellular signal can be perceived or sensed by a transmembrane protein that generates an intracellular response (Hellingwerf et al., 1998). Alternatively, the signalling molecule is transported intracellularly and its presence is then sensed by the cell, for e.g. lactose in the regulation of lac operon in Escherichia coli (see section 1.4 for details). The lac operon is induced in the presence of lactose in the media. Lactose is converted intracellularly into allolactose, which acts as an inducer of the lac operon (Pardee et al., 1959). Many of these regulatory mechanisms in prokaryotes are controlled by phosphorylation systems (Cozzone, 1988; Saier, Jr., 1993). They include:

a) ATP dependent protein kinases/phosphatases: This classical mode of phosphorylation includes enzymes that utilize alcoholic (serine and threonine) or phenolic groups (tyrosine) as acceptors to generate phosphate esters. In addition, basic amino acids (histidine, lysine and arginine) can be phosphorylated producing phosphoramidates. Furthermore, acyl groups can serve as acceptors (aspartic and glutamic acid) to generate mixed phosphate-carboxylate acid anhydrides (Cozzone, 1993). Interestingly, 
most of the bacterial kinases that belong to this class are known to be activated by intracellular metabolites (Saier, Jr., 1993). Various types of protein kinases and phosphatases commonly encountered in prokaryotes are listed in Table. 1. For any particular system, the protein kinase and protein phosphatase may be present either as two separate proteins, or unified in a single bifunctional protein (Saier, Jr., 1993). The best examples of bifunctional kinase/phosphatase are the isocitrate dehydrogenase kinase/phosphatase (AceK) from E. coli (Garnak and Reeves, 1979; LaPorte, 1993) and $\operatorname{HPr}(\mathrm{Ser}-\mathrm{P})$ kinase/phosphorylase from Firmicutes (Galinier et al., 1998; Poncet et al., 2004; Reizer et al., 1998). An example of kinase and phosphatase activities residing in separate proteins is the serine threonine kinase $\mathrm{Rbs} \mathrm{W}$ in B. subtilis, which acts in conjugation with two phosphatases $\mathrm{RbsU}$ and $\mathrm{RbsP}$ to modulate the phosphorylation state of the anti-sigma factor RbsV (Yang et al., 1996).

b) Two-component system: This type of phosphorylation system responds to an external stimulus by mediating an ATP dependent autophosphorylation at a histidyl residue of a sensor kinase. The phosphate is subsequently transferred to an aspartate residue of a second protein called the response regulator, whose activity is modulated in a phosphorylation dependent manner. Histidine protein kinases and response regulators have been identified in a wide range of Gram-positive and Gram-negative species (Fabret et al., 1999; Mascher et al., 2006). The paradigm for the two-component system is the EnvZ/OmpR in E. coli. EnvZ and OmpR are the transmembrane sensor and its cognate response regulator, respectively, that regulate the transcription of porin genes in response to medium osmolarity in E. coli (Cai and Inouye, 2002; Yoshida et al., 2002).

c) Phosphotransferases: In this system, a phosphoryl group is translocated via a set of relay proteins to the final phosphate acceptor. It involves the well characterised PEP: PTS (phosphoenolpyruvate phosphotransferase system) (Postma et al., 1993). This system is involved in the concomitant uptake and phosphorylation of a number of carbon sources. The different relay proteins receive the phosphate group at the imidazole ring of a histidine residue on either N-1 or the N-3 position. In addition, cystiene residues are phosphorylated in the PTS transport proteins (see below). This phosphorelay gives rise to high-energy bonds, providing the energy for the translocation and phosphorylation of the incoming carbon source. The PTS is regarded as a bifunctional system as besides being involved in sugar uptake, it also performs regulatory functions in the cell (discussed below in detail). 
Table. 1. Protein kinases and phosphatases in prokaryotes

\begin{tabular}{lll}
\hline Kinases family & Example/Organism & Reference \\
\hline Eukaryotic kinases homologues & AfsK from Streptomyces & Matsumoto et al., \\
& coelicolorA(3)2 & 1994 \\
Ser/Thr/Tyr specific histidine & SpoIfAB from B. subtilis & Min et al., 1993 \\
kinase homologues & & \\
Acek (Isocitrate dehydrogenase & E. coli & LaPorte and Koshland, \\
kinase/phosphatase) & & Jr., 1982 \\
HPrK/P & Firmicutes, Gram-negative & Boël et al., 2003; \\
& bacteria & Reizer et al., 1998 \\
Phosphatases family & & \\
PPP & PrpA and PrpB from E. coli & Missiakas and Raina, \\
& & 1997 \\
PPM & PrpC from B. subtilis & Obuchowski et al., \\
& & 2000 \\
Low MW PTP/Conv. PTP & YopH from & Guan and Dixon, 1990 \\
& Yersinia pseudotuberculosis & \\
\hline
\end{tabular}

${ }^{\text {a }}$ PPP (Protein phosphatase P) and PPM (Protein phosphatase M) characterized by their dependency on metal ions, inhibitor sensitivity; PTP-Protein tyrosine phosphatases.

\subsection{Sugar utilization and metabolism in B. subtilis}

\subsubsection{Carbon sources utilized by $B$. subtilis}

B. subtilis, like many other prokaryotes, can utilize a multitude of carbon sources including various complex carbohydrates such as polysaccharides composed of glucose (glycogen and starch), hemicellulosic substrates which are composed of xylose, mannose, galactose and arabinose monomers and polymers like xylan and arabinoxylans. Uptake of a little less than 20 carbon sources has already been reported for B. subtilis. These complex carbohydrates represent a major content of the plant biomass. B. subtilis secretes a large number of polysaccharide degrading enzymes, such as $\alpha$-amylase, pullulanase, endo- $\beta$-1,4-mannanase, levanase, endoglucanase and xylanase, which allow the organism to break down polysaccharides into smaller entities (Deutscher et al., 2002). Glucose in general is the most 
preferred carbon source. The various carbohydrates are subsequently taken up by different transporters that catalyze uptake of the substrates using different mechanisms.

B. subtilis employs primary and secondary active transporters, facilitators or multiprotein complexes like ATP-binding cassette (ABC) transporters and the PEP:PTS for the uptake of carbon sources (Saier et al., 2002). Facilitators mediate the energy independent uptake of substrates. Secondary active transporters utilize the energy derived from the electrochemical potential gradient of a substance across the membrane to drive transport. The symport or antiport of usually $\mathrm{H}^{+}$or $\mathrm{Na}^{+}$ions provides the energy in these cases. Primary active transporters use the hydrolysis of ATP or PEP to mediate the uptake of substrates, for e.g ABC transporter and PTS system. The PTS systems have not yet been identified in eukaryotes and archea. Uptake via PTS system introduces a phosphate in the incoming sugar, thus making the carbon source directly available for metabolism (Fig. 1 and Fig. 2).

\subsubsection{The PTS: an economical way of sugar uptake}

The PTS system is a complex multienzyme uptake system, dedicated for the concomitant phosphorylation and transport of carbohydrates across the cell membrane (Postma et al., 1993). Phosphorylation incorporates a negative charge on the sugar molecule preventing its escape from the cell.

The PTS system also provides the cell with a very economical way of sugar uptake. The phosphate is derived from PEP which has about $-14.7 \mathrm{kcal} / \mathrm{mol}$ of free energy of hydrolysis and is equivalent to one ATP in terms of energy. This allows both uptake and phosphorylation of the sugar molecule by the expenditure of only one ATP equivalent (Postma et al., 1993). In contrast, in the case of ATP transporters, 2 ATP equivalents are required for the uptake and the subsequent phosphorylation of the sugar. For this reason, many bacteria, which rely on substrate level phosphorylation for the generation of ATP (e.g. facultative anaerobic bacteria), use the PTS as the main sugar uptake system.

The PTS in general is composed of three components, namely energy coupling proteins Enzyme I (EI) ( $p t s I)$, the Histidine-containing protein ( $\mathrm{HPr}$ ) ( $p t s H)$ and several sugar specific Enzyme IIs (EII). EI and HPr are cytoplasmic proteins involved in the uptake of all PTS carbohydrates in B. subtilis. EIIs are multidomain proteins. The domains can be organised as several individual polypeptides or as a single fused protein (Meadow et al., 1990; Postma et al., 1993). The phosphoryl transfer is mediated by the EIIA and EIIB domains and the membrane bound EIIC (and EIID, if present) domain forms the sugar translocation channel. B. subtilis genome encodes 16 sugar specific EII complexes, however 
only one EI-like protein and two HPr-like proteins (HPr and Crh) are present (Reizer et al., 1999). Transport of sugars by PTS occurs with their concomitant phosphorylation (Meadow et al., 1990; Postma et al., 1993).

The overall reaction catalysed by the PTS is schematically depicted in Fig. 1. EI is autophosphorylated at histidine 189 residue using PEP as a substrate, resulting in the formation of pyruvate. Once phosphorylated, EI forms homodimers and subsequently transfers the phosphate to HPr (Dimitrova et al., 2003). The phosphate from EI is transferred to histidine 15 (His-P) residue of $\mathrm{HPr}$. HPr is a monomeric protein, which in turn phosphorylates a histidyl residue of various EIIAs. EIIB domain then receives the phosphate on a cysteyl (or histidyl) residue from EIIA and phosphorylates the sugar molecule during its translocation through the membrane spanning EIIC domain (or EIIC and EIID). The phosphorylated carbohydrate is ultimately released into the cytoplasm.

Of the two HPr like proteins, exclusively HPr participates in sugar transport. Crh (catabolite repression $\underline{H} P r$ ) does not participate in PTS. It possesses a glutamine at position 15 instead of a histidine residue, and thus cannot mediate the phosphotransfer reaction required for PTS activity (Galinier et al., 1997).

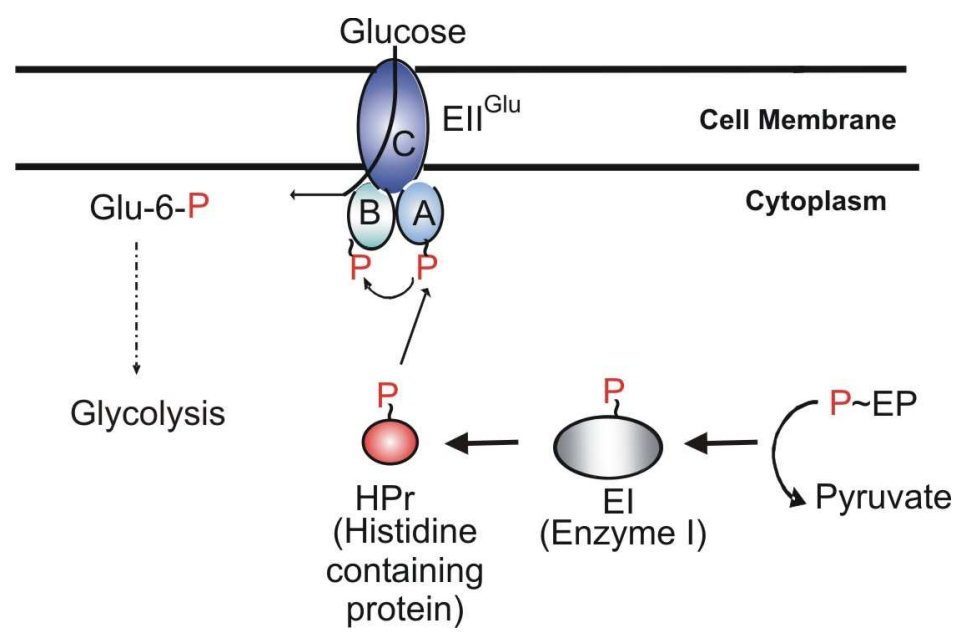

Figure. 1. The glucose specific Phosphoenolpyruvate:Phosphotranferase system (PEP:PTS) in $B$. subtilis. PEP serves as the phosphoryl donor for the phosphorylation cascade formed by the proteins EI, HPr, EIIA, and EIIB. The sugar transport step is catalysed by the membrane spanning EIIC (and EIID, if present) domain and the required energy is derived from the hydrolysis of PEP. The PTS system dedicated for the uptake of glucose in B. subtilis is depicted, in which all domains of glucose permease are fused to form a single polypeptide, leading to the arrangement, EIICBA (Postma et al., 1993; Stülke and Hillen, 2000). 


\subsubsection{General pathways for the catabolism of carbohydrates}

Bacteria convert the carbon sources utilized for growth into building blocks, reducing power and energy for maintenance and anabolism of molecules through a series and network of central metabolic reactions. The core of the carbon metabolic network is composed of three main metabolic pathways: the Embden-Meyerhoff-Parnass (EMP) pathway (or glycolysis), the pentose phosphate pathway (PPP) and the tricarboxylic acid cycle (TCA) or Krebs cycle (Fig. 2). This central metabolic core is highly conserved in most bacterial species. Thus, many steps in the metabolism of sugars are not specific to B. subtilis, but shared by a diversity of prokaryotes (Steinmetz, 1993).

B. subtilis harbours all glycolytic enzymes required for the conversion of sugar molecules starting from glucose-6-P (G6P) to pyruvate (Ludwig et al., 2001). G6P is generated during PTS mediated uptake of glucose and by the metabolism of certain disaccharides (Skarlatos and Dahl, 1998). In the next step, G6P is converted to fructose-6phosphate (F6P). F6P is then converted into fructose 1,6-bisphosphate (FBP) which is subsequently split into two C-3 molecules, dihydroxyacteone phosphate (DHAP) and glyceraldehyde-3-phosphate (see Fig. 2). Glyceraldehyde-3-P is converted to PEP by a series of enzymes. In the final step, pyruvate is generated from PEP leading to a net gain of two ATP and two NADH molecules. Pyruvate then enters the Krebs cycle.

Sugars like D-fructose, D-mannitol, sorbitol, myo-inositol, glucosamine and glycerol are catalytically converted to various intermediates of glycolysis. Pentoses including arabinose, ribose and xylose are metabolised via the pentose phosphate (PPP) pathway. Gluconate is also metabolised via the PPP as B. subtilis lacks the Entner Doudoroff pathway, which is utilized by various bacteria like Enterococcus faecalis to catabolize gluconate. Metabolic routes required for the assimilation of various sugars and their uptake systems are depicted in the figure 2. Depending on the carbon source used, the flux through this central metabolic network is differentially distributed. For example, when glucose or glycerol is utilized as the sole carbon source, the flux through the initial steps of glycolysis is not similar. When a carbon source that feeds into the Krebs cycle is used as the sole carbon source, the flux through the glycolytic steps is in the reverse order to generate metabolites like F6P and G6P, the building blocks for anabolism (gluconeogenesis).

The various reactions in the central metabolism of B. subtilis are highly capable of adapting to the availability of carbon sources and to their different concentrations. Diverse mechanisms including allosteric control of enzymes and transcriptional regulation are involved in achieving these regulations (Stülke and Hillen, 2000). 


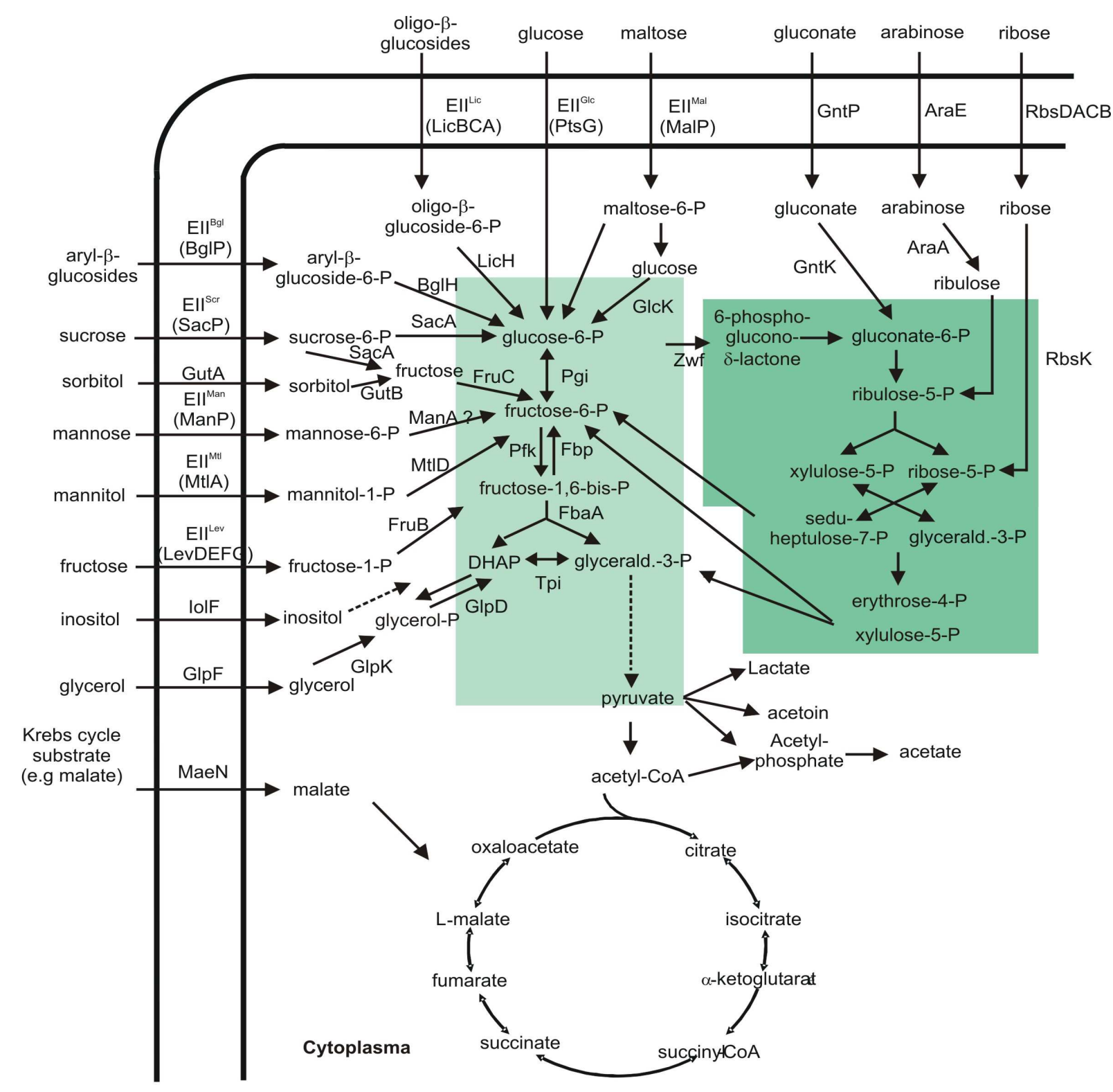

Figure. 2. Schematic representation of the transport systems and the metabolic routes required for the uptake and assimilation of various carbon sources which can be utilized by $B$. subtilis. Both PTS and non-PTS transport systems are represented. For sugars that are taken up by the PTS system, respective EIIs are depicted. Glycolytic pathway is indicated in light green and pentose phosphate pathway in dark green. The various enzymes involved in metabolic pathways connecting the assimilated carbon source and its subsequent entry into the central metabolic core are also shown. LicH, 6-phospho- $\beta$-glucosidase; BglH, 6-phospho- $\beta$-glucosidase; SacA, sucrose-6-P-hydrolase; FruC, fructokinase; GutB, glucitol dehydrogenase; ManA, mannose-6-P-isomerase; MtlD, mannitol-1-Pdehydrogenase; FruB, 1-phosphofructokinase; GlpK, glycerol kinase; GlpD, glycerol dehydrogenase, Pgi, glucose-6-P-isomerase; Pfk, 6-phosphofructokinase; Fbp, fructose-1,6-bisphosphatase; Fba, fructose-1,6-bisphosphate aldolase; Tpi, triose-phosphate-isomerase; Zwf, gluconate-6-Pdehydrogenase; GlcK, glucose kinase; GntK, gluconate kinase; Ara, L-arabinose isomerase; RbsK , ribokinase (adapted and modified from Görke and Deutscher, 2007). 


\subsection{Regulation of carbon metabolism}

Usually bacteria differentially regulate gene expression, such that only the genes involved in the utilization of the available carbon sources are switched on. In the absence of a substrate, the corresponding subsets of genes are repressed. This mechanism is defined as induction and in B. subtilis it is achieved by various regulatory mechanisms mainly at the level of transcription of various catabolic operons (Steinmetz, 1993). Different operons are employ different mechanisms to be induced in the presence of their respective substrates. The regulation of $l a c$ operon in $E$. coli is a classical example of inducible gene expression. The $l a c$ operon is involved in the transport and metabolism of lactose in E. coli and consists of three structural genes $l a c Z$, lac $Y$ and $l a c A$ encoding for $\beta$-galactosidase, $\beta$-galactosidase permease and $\beta$-galactosidase transacetylase, respectively. The operon is under the negative control of the lactose repressor encoded by the lacI gene. In the absence of the inducer lactose, LacI binds to the operator sequence preceding the operon genes and represses the expression of the operon. When cells are grown in the presence of lactose, a lactose metabolite called allolactose is generated intracellularly, which when bound to LacI, prevents the repression of the operon (Pardee et al., 1959). Similarly, the expression of the xyn and xyl operons, which are involved in the metabolism and uptake of xylose, in B. subtilis are induced in the presence of the substrate xylose (see chapter 3 and 5).

When exposed to a multitude of alternative nutrients, cells respond in a more complex manner. They selectively take up the carbon source that provides them with the most energy and growth advantage. Simultaneously, they repress the various functions involved in the catabolism and uptake of less preferred carbon sources. This phenomenon is commonly referred as carbon catabolite repression (CCR) (Deutscher, 2008; Görke and Stülke, 2008). CCR was first described in B. subtilis by Jacques Monod in 1942. Monod observed a hierarchical utilization of various carbon sources, glucose being the most preferred carbon source (Monod, 1942). He classified the carbon sources utilized by B. subtilis in two groups, $\mathrm{A}$ and $\mathrm{B}$. When present in a mixture, the bacteria utilize preferentially the substrates of group A, which include glucose, fructose and mannitol. After a delay of growth, the cells make use of the group B carbon sources such as sorbitol, arabinose and maltose. This biphasic growth was termed as diauxie (Monod, 1942). Extended studies on this phenomenon established glucose as the preferred carbon source for many organisms.

Repression of utilization of secondary carbon sources can be mediated via several mechanisms. These mechanisms have been studied in detail in both, Firmicutes such as B. subtilis and Enterobacteriaceae such as E. coli (Deutscher, 2008; Görke and Stülke, 2008). 
Even though the main regulatory mechanisms operative in these bacteria are quite different, they mostly employ components of the PEP:PTS. In general, repression of utilization of a secondary carbon source can be achieved by the following mechanisms (Görke and Stülke, 2008; Stülke and Hillen, 2000):

a) The intracellular formation of the specific inducer of catabolic operons can be prevented (inducer exclusion) in the presence of a preferred carbon source.

b) The activity of the operon specific regulatory proteins can be altered, leading to an anti induction effect (induction prevention).

c) A number of catabolic operons can be regulated/repressed by employing a global regulator in response to the availability of the preferred carbon source.

\subsubsection{The regulatory role of PTS in CCR: linking sugar uptake and metabolism.}

\subsubsection{CCR in E. coli}

All phospho transfer events during PTS transport activity are reversible, except for the transfer of the phosphoryl group to the sugar molecule. Thus, at any given time the phosphorylation state of PTS proteins depends on the presence of a PTS substrate and the metabolic state of the cell i.e. the PEP:pyruvate ratio (Görke and Stülke, 2008; Hogema et al., 1997; Hogema et al., 1998). The varying phosphorylation state of the PTS proteins provides the cell with a sensory mechanism to sense the availability of preferred carbon sources in the medium.

In E. coli, EIIA ${ }^{\text {glu }}$ is the master regulator of carbon catabolite repression. In the presence of glucose or other rapidly metabolizable substrate, EIIA ${ }^{\text {glu }}$ is mainly present in the dephosphorylated form since the phosphate is directed towards the incoming sugar. Unphosphorylated EIIA ${ }^{\text {glu }}$ can interact with transport systems of several non-PTS sugars for e.g. lactose, maltose, mellibiose and inhibits their activity, excluding the inducer of the respective catabolic operon from the cell (Görke and Stülke, 2008; Saier, Jr. and Roseman, 1976). Inducer exclusion is one of the two main mechanisms for catabolite repression in E. coli. Various non PTS substrates like G6P or lactose can also exert CCR by modulating the PEP:pyruvate ratio by regulating the flux through glycolysis (Hogema et al., 1997; Hogema et $a l .$, 1998). In the presence of a rapidly metabolizable sugar, PEP:pyruvate ratio decreases in the cell. The decrease in the intracellular concentration of PEP, the phospho donor of PTS, eventually leads to unphosphorylated EIIA ${ }^{\text {glu }}$.

On a global level, an additional mechanism exists in E. coli. In the absence of glucose, EIIA $^{\text {glu }}$ is phosphorylated and activates adenylate cyclase (Cya) by binding to it. This leads to 
an increase in intracellular cyclic AMP (cAMP) levels. The transcriptional activator Crp (cAMP receptor protein) is the global regulator of CCR in E. coli and requires cAMP to be functionally active. Thus in the presence of glucose, $\operatorname{Crp}$ is inactivated and various catabolic operons under the control of Crp/cAMP can not be expressed (Fig. 3) (Görke and Stülke, 2008; Gosset et al., 2004; Martinez-Antonio and Collado-Vides, 2003).

activation of $b g l$ operon

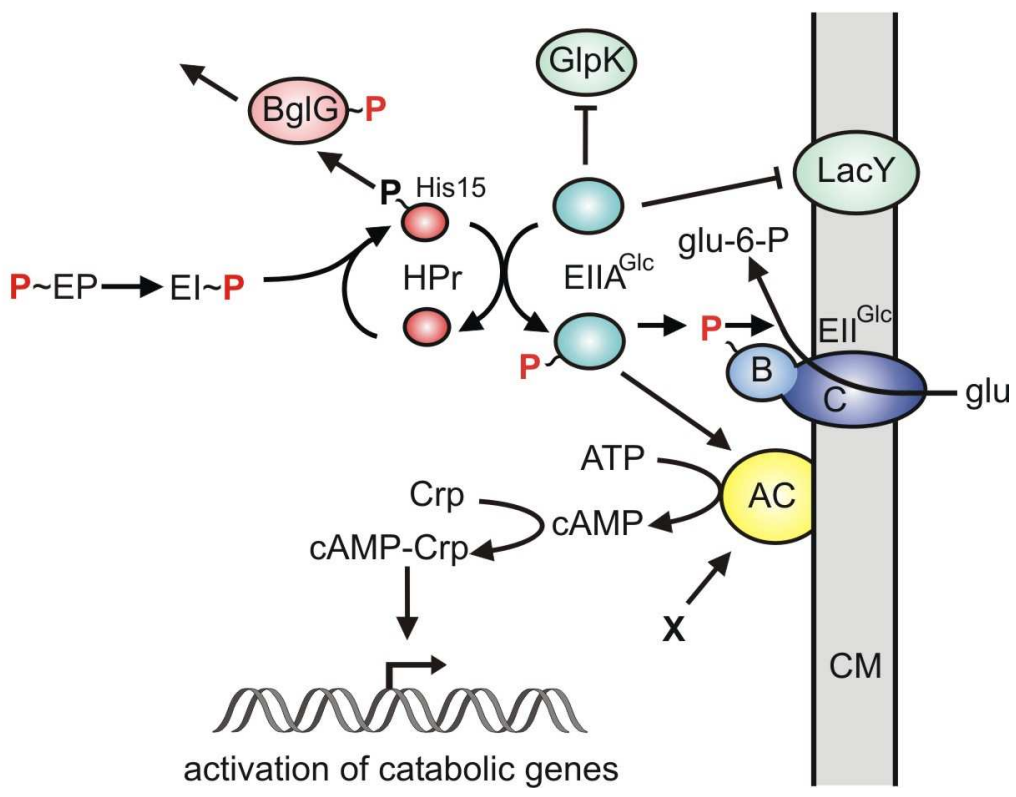

Figure. 3. CCR in E. coli. In the presence of glucose, EIIA ${ }^{\text {glu }}$ is unphosphorylated and in this form it interacts with transporters of secondary carbon sources and metabolic enzymes and inactivates them. In the absence of glucose, EIIA ${ }^{\text {glu }}$ retains its phosphate and in this state it binds and activates adenylate cyclase (AC), which leads to cyclic AMP (cAMP) synthesis. An unknown 'factor $\mathrm{x}^{\prime}$ is also required for the activation of AC. High cAMP concentrations trigger the formation of cAMP-CRP complexes, which bind and activate the promoters of catabolic genes (adapted from Görke and Stülke, 2008).

\subsubsection{CCR in B. subtilis}

In Firmicutes like B. subtilis, the cAMP dependent pathway of catabolite repression does not exist. In contrast, the PTS phosphocarrier protein HPr is the key regulator of CCR in these organisms. Unlike in Enterobacteriaceae, HPr in Firmicutes can also be phosphorylated at the Serine 46 residue in an ATP dependent reaction catalysed by a bifunctional enzyme called HPrK/P (Deutscher and Saier, 1983; Reizer et al., 1998). However, both forms of HPr, $\mathrm{HPr}($ His-P) and $\mathrm{HPr}($ Ser-P), are involved in CCR.

Inducer exclusion also exists in Firmicutes. Repression of glycerol metabolism in the presence of a rapidly metabolizable carbon source is a well investigated example of inducer exclusion regulated by $\mathrm{HPr}(\mathrm{His}-\mathrm{P})$ in $B$. subtilis. Glycerol is taken up by the facilitator protein, GlpF and converted to glycerol-3-phosphate (G3P) in an ATP dependent manner by 
the glycerol kinase, GlpK (Pettigrew et al., 1988). G3P serves as the inducer for the glpFK operon and functions by activating the operon specific antiterminator protein in B. subtilis called, GlpP (Darbon et al., 2002; Rutberg, 1997). The glycerol kinase is activated by HPr(His-P) dependent phosphorylation. Thus, in the presence of a PTS sugar, HPr(His-P) donates the phosphate to an incoming sugar rather than activating the glycerol kinase. This prevents the intracellular generation of the inducer G3P and ultimately the repression of the glpFK operon (Darbon et al., 2002). Due to this reason, mutations in either of the general PTS proteins abolish growth of the bacterium on glycerol as the sole carbon source (Beijer and Rutberg, 1992; Reizer et al., 1984). Inducer exclusion mediated by $\operatorname{HPr}(\operatorname{Ser}-\mathrm{P})$ has also been reported in some organisms. In Lactobacillus brevis, $\mathrm{HPr}(\mathrm{Ser}-\mathrm{P})$ has been suggested to bind the galactose $/ \mathrm{H}^{+}$symporter, GalP and inhibit galactose uptake in the presence of glucose (Ye and Saier, Jr., 1995). Similarly in L. casei and L. lactis, presence of glucose inhibits maltose uptake at the transport step itself, thus repression being achieved by $\operatorname{HPr}(\operatorname{Ser}-\mathrm{P})$ (Dossonnet $e t$ al., 2000; Viana et al., 2000).

Another interesting mode of catabolite repression is by antagonising the positive activity of various operon specific regulators. This mechanism called induction prevention exists in a variety of bacteria, e.g. in E. coli and B. subtilis. One of the interesting examples of this mode of repression is one which is mediated by PTS. Various catabolic genes require transcriptional regulators which contain conserved regulatory domains that can be phosphorylated by PTS proteins (PTS regulatory domains, PRDs) (Stülke et al., 1998) (Fig. 4). These regulators can act as transcription antiterminators or transcription activators and are active only in the presence of specific inducers. PRDs usually contain two histidyl residues which are phosphorylated by either $\mathrm{HPr}(\mathrm{His}-\mathrm{P})$ or $\mathrm{EIIB}(\mathrm{P})$ (Deutscher et al., 2006; Görke and Deutscher, 2007). Most PRD containing proteins have two PRDs. In B. subtilis, there are four PTS controlled antiterminator proteins regulating the transcription of several genes. GlcT and SacY control the expression of the $p t s G(H I)$ and $s a c B$ operon, respectively (Crutz et al., 1990; Görke and Deutscher, 2007; Schmalisch et al., 2003; Stülke et al., 1997). SacT and LicT control the expression of $s a c P A$ and $b g l P H$ operon, respectively (Aymerich and Steinmetz, 1992; Krüger and Hecker, 1995). These antiterminator proteins have the similar domain organisation containing PRD-1 and PRD-II. The PRD-I can be negatively phosphorylated by their specific EIIs in the absence of the cognate substrate and rendered inactive. The PRD-II can be positively phosphorylated by HPr(His-15). In the presence of glucose or any other rapidly metabolizable carbon source, such antiterminators compete for the limited pool of $\mathrm{HPr}(\mathrm{His}-\mathrm{P})$ with EIIAs. Thus the activating phosphorylation by 
HPr(His-P) is limited. In vitro experiments suggest that the rate of transfer of phosphate is faster towards EIIAs and thus antiterminators are rendered functionally inactive (Lindner et al., 1999). The antiterminator BglG from E. coli is also regulated by the antagonistic phosphorylation on PRD-I and PRD-II. BglG modulates the transcriptional antitermination of the $b g l$-operon and is negatively regulated by phosphorylation at PRD1 by its cognate EII and activated by phosphorylation by $\operatorname{HPr}($ His-P) (Görke and Rak, 1999) (Fig. 3).

Interestingly, the PRD-II of GlcT and SacY can also be phosphorylated by HPr, but the activity of GlcT and SacY is not severly influenced by the phosphorylation state of PRD-II (Bachem and Stülke, 1998; Schmalisch et al., 2003; Tortosa et al., 1997).

\subsubsection{Global mechanism of carbon catabolite repression in B. subtilis}

\subsubsection{Role of HPr(Ser-P) and CcpA}

As mentioned before, HPr is the key regulator of catabolite repression in Firmicutes. In these organisms HPr can be phosphorylated at serine 46 residue. The regulatory role of HPr(Ser-P) in catabolite repression in Firmicutes was initially identified in Streptococcus pyogenes. In this organism $\mathrm{HPr}(\mathrm{Ser}-\mathrm{P})$ is involved in inducer exclusion of several secondary carbon sources when the cells grow in the presence of glucose (Deutscher and Saier, Jr., 1983). In 1994, the first report that identified $\operatorname{HPr}(\operatorname{Ser}-\mathrm{P})$ as a global regulator in catabolite repression was published. Deutscher and co-workers demonstrated that in a B. subtilis ptsHI (serine 46 to alanine replacement) mutant, a number of catabolic enzymes are only partially or not at all repressed by glucose (Deutscher et al., 1994).

Later on, a pioneering work on CCR of $\alpha$-amylase synthesis in B. subtilis led to the identification of another important player in CCR mechanism: the DNA binding protein called CcpA (carbon catabolite protein $\underline{\text { A) }}$ (Henkin et al., 1991). Global expression studies using proteomics and transcriptomics approaches demonstrated that the repression of a number of a similar set of genes is relieved in the presence of glucose in a ccpA as well as in a ptsH1 mutant, implying that they both contribute to the same repression mechanism (Lorca et al., 2005).

The CcpA protein is a member of the LacI/GalR family of transcriptional regulators. Independent approaches identified a specific interaction between CcpA and HPr(Ser-P). Both retardation elution experiments and nuclear magnetic resonance spectroscopy demonstrates that $\operatorname{HPr}(\operatorname{Ser}-\mathrm{P})$ specifically forms a complex with CcpA (Jones et al., 1997). Interestingly, only the serine phosphorylated form of HPr can interact with CcpA. This phosphorylation increases the binding affinity of CcpA for HPr over 50 folds, indicating the importance of 
serine phosphorylation for the specificity and affinity of this interaction (Jones et al., 1997). The inability of $\mathrm{HPr}(\mathrm{His}-\mathrm{P})$ to interact with $\mathrm{CcpA}$ is explained by the crystal structure available for the ternary complex of CcpA/HPr(Ser-P)/cre. Histidine 15 of $\mathrm{HPr}$ is engaged in hydrogen bonding with an aspartate residue of $\mathrm{CcpA}$ and thus introduction of phosphate at this residue causes steric hindrances for its interaction with CcpA (Schumacher et al., 2004).

The CcpA protein forms a dimer of two identical subunits and consists of an $\mathrm{N}$-terminal DNA binding domain and a large C-terminal oligomerization and effector binding domain (Jones et al., 1997; Schumacher et al., 2004).

The interaction of HPr(Ser-P) with CcpA stimulates its DNA binding ability and the complex binds to a conserved 14 nucleotide long imperfect-palindromic DNA sequence called catabolite repressive element (Aung-Hilbrich et al., 2002; Seidel et al., 2005; Weickert and Chambliss, 1990). These regulatory cre sites occur in single or multiple copies in different catabolic operons at different positions with respect to the promoter. Repression by CcpA usually requires cre sequences to be located downstream of the promoter or overlapping with the promoter in front of the structural genes (Miwa et al., 1997). Roughly $10 \%$ of the genome is under the control of CcpA mediated regulation and the majority of these genes are repressed by CcpA. Most of the genes regulated by $\mathrm{CcpA}$ encode proteins involved in utilization of secondary carbon sources (Blencke et al., 2003; Moreno et al., 2001; Yoshida et al., 2001).

In certain cases, cre sites are present upstream of the promoter region. In such cases the transcription units are subjected to activation by CcpA. The genes involved in overflow metabolism (pta, ackA, alsSD) are induced in the presence of glucose in a CcpA/cre dependent manner (Lorca et al., 2005). Interestingly, genes lacking a functional cre site can still be regulated by CcpA, for example, the expression of the gapA operon. The gapA operon includes the glycolytic genes gapA, pgk, tpi, pgm and eno encoding for glyceraldehyde-3phosphate dehydrogenase, phosphoglycerate kinase, triose phosphate isomerase, phosphoglycerate mutase and enolase, repectively (Kunst et al., 1997; Leyva-Vazquez and Setlow, 1994). This mode of regulation of gapA operon is achieved by a novel mechanism. For unknown reasons, a ccpA mutant contains larger amounts of $\operatorname{HPr}(\operatorname{Ser}-\mathrm{P})$ than the wild type in B. subtilis and E. faecalis (Leboeuf et al., 2000; Ludwig et al., 2002). This increased percentage of $\mathrm{HPr}(\mathrm{Ser}-\mathrm{P})$ slows down the glucose uptake, as the substrate for PTS transport, $\mathrm{HPr}($ His-P), is not available in the required amounts. In the absence of glycolytic substrates like glucose, expression of the gapA operon is repressed by a transcriptional repressor called CggR. On the other hand, CggR is inactivated by elevated amounts of FBP, which explains 
why the activation of gapA operon is not functional in a $c c p A$ mutant strain (Doan and Aymerich, 2003; Zorrilla et al., 2007).

\subsubsection{Role of Crh in CCR in B. subtilis}

Although most catabolite repressed genes are relieved from catabolite repression in ccpA and ptsH1 mutant, the degree of relief is found to be quite different for some genes and operons for the two mutants. For example the amyE, hut, cta, xyn and iol operons are only partially relieved from glucose exerted CCR in a ptsH1 mutant, whereas it is completely relieved in ccpA mutant (Deutscher et al., 1994; Galinier et al., 1997; Voskuil and Chambliss, 1996; Zalieckas et al., 1999). Thus, a second co-repressor for CcpA should account for these differences. Crh, a protein which exhibits $45 \%$ sequence identity to HPr was found to be this co-repressor. Similar to HPr, Crh can be phosphorylated at serine 46 residue by HPrK/P and this phosphorylated form interacts with CcpA. However, biochemical studies indicate that $\mathrm{Crh}(\operatorname{Ser}-\mathrm{P})$ binds CcpA with an up to 10 fold reduced affinity as compared to $\mathrm{HPr}(\operatorname{Ser}-\mathrm{P})$. The reason for this low affinity has been attributed to weaker interaction of one of its contact region to CcpA (Schumacher et al., 2006).

The genes, which are partially relieved in a ptsH1 mutant, are completely relieved in a ptsH1 crh double mutant. Although these in vivo and in vitro results unequivocally establish that $\mathrm{Crh}(\mathrm{Ser}-\mathrm{P})$ can contribute to $\mathrm{CCR}$ in B. subtilis, some observations question this role of Crh. For example, if only $c r h$ gene is disrupted, no effect on CCR is observed (Galinier et al., 1997).

\subsubsection{HPrK/P: The central player of CCR}

HPrK/P plays a key role in CCR in the Firmicutes. It catalyses the first step of the global carbon catabolite repression response i.e the phosphorylation of $\mathrm{HPr}$ and $\mathrm{Crh}$ at their serine 46 residues. HPrK/P exhibits no similarity to eukaryotic Ser or Thr protein kinases. In contrast, it possesses a nucleotide binding motif called P-loop (or Walker A motif) present in many ATPase or GTPase activity exhibiting proteins, nucleotide binding proteins, ABC transporters, proteases and chaperons. The crystal structure of HPrK/P from three organisms Staphylococcus xylosus, Mycoplasma pneumoniae and a truncated version from Lactobacillus casei reveals that HPrK/P is a hexamer in the cell with subunits folding into two distinct structural domains. The C-terminal domain carries both kinase and phosphorylase activity, whereas the $\mathrm{N}$-terminal domain has no defined function, yet. The C-terminal domain contains the active site, the Walker A motif forming the phosphate binding loop (P-loop) of the nucleotide binding site is composed of an N-terminal domain, followed by an active site Walker A (Allen et al., 2003; Fieulaine et al., 2002; Marquez et al., 2002). One hexamer 
binds six HPr molecules on its surface. The crystal structure of the complex of the truncated version of $L$. casie, revealed that binding of one HPr molecule occurs via two interface regions formed by one HPr molecule and two adjacent HPrK/P subunits located within one trimer of the hexamer (Poncet et al., 2004).

$\mathrm{HPrK} / \mathrm{P}$ has been designated as a bifunctional enzyme as it can carry out both the phosphorylation and dephosphorylation of $\mathrm{HPr}(\mathrm{Ser}-\mathrm{P})$ and $\mathrm{Crh}(\mathrm{Ser}-\mathrm{P})$ in vitro. It was found that the presence of inorganic phosphate $\left(\mathrm{P}_{\mathrm{i}}\right)$ stimulates dephosphorylation of these substrates. Indeed, the structural analysis suggests that the P-loop motif of HPrK/P can bind not only ATP but also $P_{i}$ at the same position as the $\beta$-phosphate of the nucleotide (Fieulaine et al., 2001). Thus, a dephosphorylation reaction mechanism in which inorganic phosphate carries out a nucleophilic attack on the phosphoryl group in $\operatorname{HPr}(\operatorname{Ser}-\mathrm{P})$ has been proposed. The result of this dephosphorylation reaction is the generation of pyrophosphate $\left(\mathrm{PP}_{\mathrm{i}}\right)$. This phosphorylase reaction is reversible and HPrK/P can use $\mathrm{PP}_{\mathrm{i}}$ to phosphorylate HPr in vitro. Thus, HPrK/P can theoretically use both ATP and $\mathrm{PP}_{\mathrm{i}}$ as a phospho donor (Mijakovic et al., 2002).

\subsubsection{Interplay of protein factors (HPr, Crh, HPrK/P and CcpA) and small molecule effectors: a model to describe CCR in B. subtilis}

One of the consequences of carbon source utilization and assimilation is a change in the metabolic state of the cell where the energy charge (ATP/ADP ratio) and the intracellular concentrations of various metabolites changes. Most of the metabolic enzymes and transcriptional regulators involved in carbon metabolism are allosterically regulated and their activity is modulated by binding of small effector molecules (Saier, Jr., 1993). CCR in Firmicutes and in Enterobacteriacea is also affected by various metabolic intermediates besides the protein factors described above.

So far catabolite repression has always been described with respect to glucose as the repressing sugar. Glucose is known to generate high amounts of intracellular HPr(Ser-P). Approximately $60 \%$ of the total HPr in B. subtilis is phosphorylated at its serine residue and a very little fraction is observed to be histidine phosphorylated (Ludwig et al., 2002; Monedero et al., 2001).

It has been reported that both the kinase and the phosphorylase activity of $B$. subtilis HPrK/P can be allosterically regulated. A number of metabolic intermediates were screened 


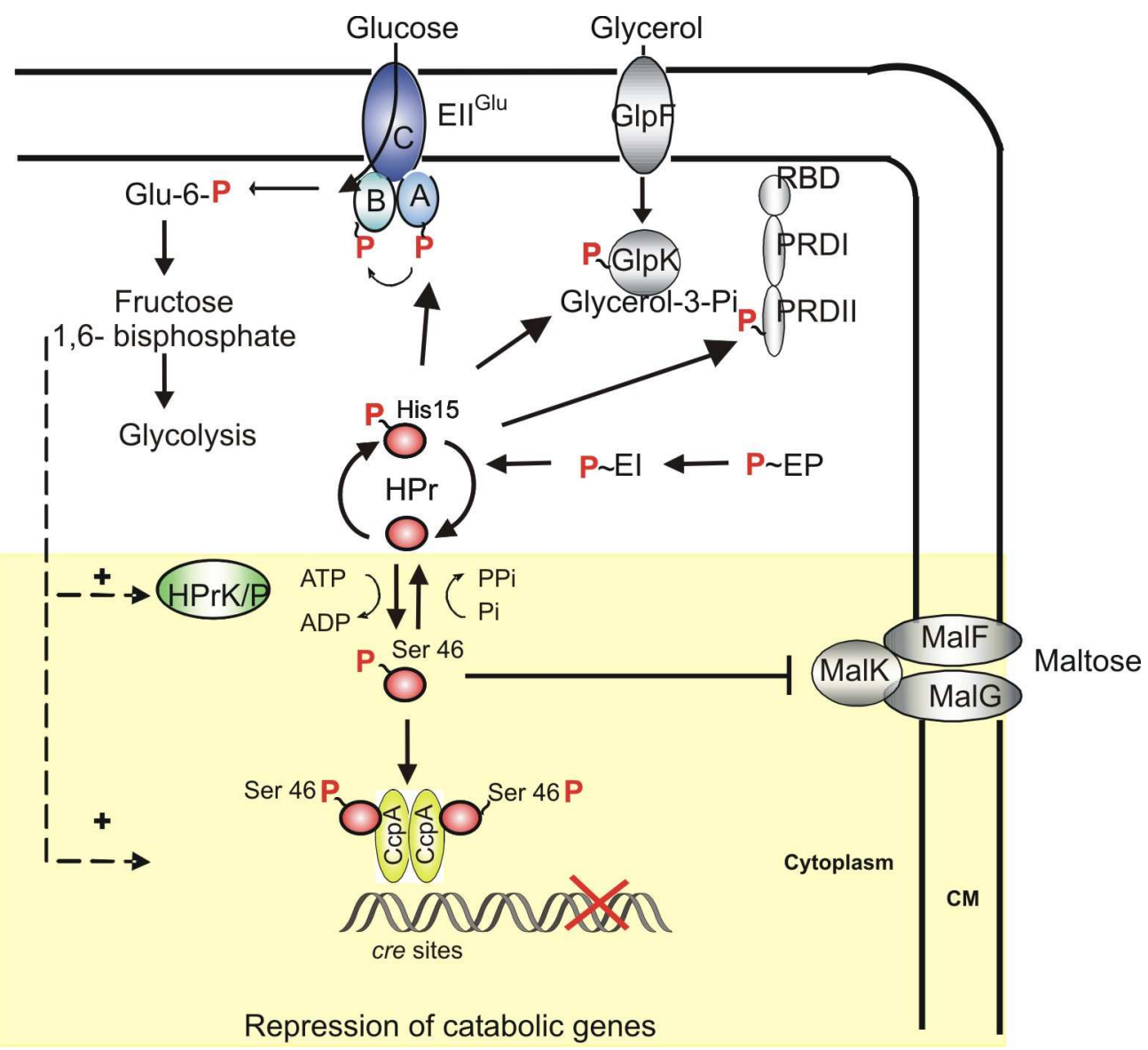

Figure. 4. Modes of catabolite repression in low G+C content Gram-positive bacteria (Görke and Stülke, 2008). In the presence of a rapidly metabolizable carbon source like glucose, the intracellular concentration of metabolites like FBP increases, leading to an allosteric activation of kinase activity of HPrK/P and ultimately to the generation of $\mathrm{HPr}(\mathrm{Ser}-\mathrm{P})$. Serine phosphorylated $\mathrm{HPr}$ interacts with CcpA dimer (two HPr molecules). The protein complex binds to the operator sequences, cre sites and represses the transcription units bearing cre sites overlapping with or downstream of their promoter region. $\operatorname{HPr}(\mathrm{Ser}-\mathrm{P})$ can also interact with transporters, inhibiting the intracellular accumulation of secondary carbon sources (illustrated in the part of the figure highlighted in yellow colour). The presence of glucose leads to a net dephosphorylation of PTS proteins including HPr(HisP). This low level of HPr(His-P) does not allow sufficient phosphorylation dependent activation of GlpK and PRD bearing proteins like LicT in B. subtilis. In some Gram-positive bacteria, HPr(Ser-P) also mediates inducer exclusion of secondary substrates by interacting with their transporter proteins. For e.g. in L. casie and L. lactis, $\operatorname{HPr}(\mathrm{Ser}-\mathrm{P}$ ) is suggested to bind to MalK (adapted and modified from Görke and Stülke, 2008).

and it was shown in vitro that FBP can stimulate the ATP dependent kinase activity of HPrK/P (Jault et al., 2000). In this context, the intracellular concentration of FBP, ATP and $\mathrm{P}_{\mathrm{i}}$ vary largely depending on whether the cell utilized a favourable carbon source or not. $B$. subtilis cells grown in the presence of glucose contain 10 fold more FBP (from 1.4 mM to 14 $\mathrm{mM}$ ) and $\mathrm{PP}_{\mathrm{i}}(1.2 \mathrm{mM}$ to $6 \mathrm{mM}$ ) than cells grown on succinate (Mijakovic et al., 2002). On the other hand when cells are starved, concentration of inorganic phosphate increases drastically as observed in the case of Streptococcus bovis (upto $30 \mathrm{mM}$ ) (Asanuma and Hino, 2003). 
Interaction of $\operatorname{HPr}(\operatorname{Ser}-\mathrm{P})$ and $\mathrm{CcpA}$ has also been shown to be affected by various metabolites. Structural studies using Bacillus megaterium CcpA and HPr(Ser-P) demonstrated that the presence of FBP and G6P bolster cross interaction between arginine 17 in $\mathrm{HPr}(\mathrm{Ser}-\mathrm{P})$ and the aspartate residues 69 and 99 of the CcpA subunit. This further stabilizes the closed DNA binding conformation of CcpA and enhances the interaction of the CcpA-HPr(Ser-P) complex with its cognate target DNA (Schumacher et al., 2007).

For B. subtilis gnt cre site and B. megaterium xyl cre site, G6P has also been shown to allow CcpA binding to cre independent of $\mathrm{HPr}(\operatorname{Ser}-\mathrm{P})$. However, these effects were observed only under non physiological conditions such as very high concentrations of G6P for gnt cre and low $\mathrm{pH}$ for $x y l$ cre (Miwa et al., 1997).

Based on the above observations, a general CCR mechanism has been proposed for B. subtilis and other Firmicutes. In the presence of a rapidly metabolizable carbon source such as glucose, an increases in FBP in cells stimulates the HPrK/P catalyzed formation of $\mathrm{HPr}($ Ser-P), resulting in the formation of a complex between $\mathrm{HPr}(\operatorname{Ser}-\mathrm{P})$ and CcpA and this repressor complex then binds to the cre sites (Fig. 4) (Görke and Stülke, 2008).

\subsection{Aim of this work}

CCR can be defined as the repression of functions required for the utilization of secondary carbon sources, in the presence of a preferred and rapidly metabolized carbon source. Glucose is often the preferred carbon source for many organisms. Catabolite repression mediated by glucose has been extensively studied in the model organisms E. coli and B. subtilis. In E. coli, the repressing potential of various other carbon sources besides glucose has also been studied and it was shown that a large number of carbohydrates in addition to glucose exert CCR. In B. subtilis most of the available knowledge regarding CCR is derived from experiments using glucose as the repressing carbon source. One of the aims of this work was to systematically study the repressing potential of various carbon sources besides glucose. It was also required to understand if the ability of a carbon source to cause CCR is related to its uptake mechanism and/or the catabolic pathway of a given substrate. To analyse this, carbon sources which differ from glucose in their chemical nature and their uptake mechanisms, were used to study CCR in B. subtilis. During this work, the factors contributing to CCR and the phosphorylation state of HPr were analysed.

A further aim of this work was to analyse the role of PrpC in CCR. PrpC is a Ser/Thr phosphatase and dephosphorylates $\mathrm{HPr}(\operatorname{Ser}-\mathrm{P})$ in $M$. pneumoniae. 


\section{List of publications}

Singh,K.D., Halbedel,S., Görke,B., and Stülke,J. (2007) Control of the phosphorylation state of the HPr protein of the phosphotransferase system in Bacillus subtilis: implication of the protein phosphatase PrpC. J Mol Microbiol Biotechnol 13: 165-171.

Singh,K.D., Schmalisch,M.H., Stülke,J., and Görke,B. (2008) Carbon Catabolite Repression in Bacillus subtilis: A Quantitative Analysis of Repression Exerted by Different Carbon Sources. J Bacteriol. DOI: 10.1128/JB.00848-08 
2. Control of the Phosphorylation State of the HPr Protein of the Phosphotransferase System in Bacillus subtilis: implication of the Protein Phosphatase PrpC

The results described in this chapter were published in:

Singh,K.D., Halbedel,S., Görke,B., and Stülke,J. (2007) Control of the phosphorylation state of the HPr protein of the phosphotransferase system in Bacillus subtilis: implication of the protein phosphatase PrpC. J Mol Microbiol Biotechnol 13: 165-171.

Author contribution:

This study was designed and interpreted by Kalpana Singh, Boris Görke and Jörg Stülke. All experiments were performed by Kalpana Singh. Sven Halbedel contributed in suggesting the role of PrpC in M. pneumoniae. This paper was written by Jörg Stülke and Kalpana Singh. 


\begin{abstract}
In the Gram-positive bacterium Bacillus subtilis as well as in other Firmicutes, the HPr protein of the phosphotransferase system (PTS) has two distinct phosphorylation sites, His-15 and Ser-46. These sites are phosphorylated by the Enzyme I of the PTS and by the ATPdependent HPr kinase/ phosphorylase, respectively. As a result, the phosphorylation state of HPr reflects the nutrient supply of the cell and is in turn involved in several responses at the levels of transport activity and expression of catabolic genes. Most important, $\mathrm{HPr}(\mathrm{Ser}-\mathrm{P})$ serves as a cofactor for the pleiotropic transcription regulator CcpA. In addition to the proteins that phosphorylate $\mathrm{HPr}$, those that are involved in the dephosphorylation are important in controlling the overall HPr phosphorylation state and the resulting regulatory and physiological outputs. In this study, we found that in addition to the phosphorylase activity of the HPr kinase/ phosphorylase the serine/ threonine protein phosphatase PrpC uses HPr(SerP) as a target.
\end{abstract}

\title{
Introduction
}

In order to respond to changes in the environmental conditions or in the supply of nutrients, all living cells are capable of sensing such changes and of transducing the corresponding signals in a way that allows an adaptation of the cell. The signal transduction processes often involve covalent modifications of proteins that result either in changes of enzymatic activity or affect the cellular gene expression programme. Among these modifications protein phosphorylation is the most prominent one since it can affect the activity of a protein drastically due to the large size of the phosphate group and its strong negative charge (Huffine and Scholtz, 1996; Johnson and Barford, 1993; Kennelly and Potts, 1996).

In bacteria, three major types of signal transduction systems are controlled by reversible phosphorylation. These include the two-component systems, the stressosome regulatory systems involving sigma factors and anti-sigma factors, and the phosphoenolpyruvate:sugar phosphotransferase system-derived regulatory systems (PTS) (Pané-Farré et al., 2005; Postma et al., 1993; Stock, 2000; Stülke et al., 1998). In each case, phosphorylation results in the reversal of the biological activity of the phosphorylated protein and thus in a signalling process.

The PTS was discovered as a sugar transport system that couples the transport of a substrate to its concomitant phosphorylation (Postma et al., 1993). The phosphate group is 
derived from phosphoenolpyruvate and is transferred via the two general energy-coupling proteins Enzyme I and HPr and the A and B domains of the sugar permease (IIA and IIB) to the incoming sugar. In Escherichia coli, the phosphorylation state of the glucose-specific IIA controls the synthesis of the signalling molecule cAMP and the activity of a set of sugar transporters (see Postma et al., 1993 for review). In many other bacteria, HPr rather than the IIA protein is crucial for signal transduction. In the low GC-branch of the Gram-positive bacteria (i.e., the Firmicutes) and in many Gram-negative bacteria (with the notable exception of the enteric bacteria), HPr is not only phosphorylated by Enzyme I of the PTS at a histidine residue at position 15 (His-15) but also by a metabolite-controlled kinase called HPr kinase/phosphorylase (HPrK/P) on Ser-46 (Boël et al., 2003; Poncet et al., 2004).

In Bacillus subtilis, $\mathrm{HPr}(\mathrm{His}-\mathrm{P})$ is present in cells grown in the absence of preferred carbon sources such as glucose (Ludwig et al., 2002; Monedero et al., 2001). This form of HPr is involved in sugar transport by the PTS and can transfer its phosphate to glycerol kinase and several transcriptional regulators to stimulate their activity (Darbon et al., 2002; Stülke et al., 1998). If glucose is present in the medium, the HPrK/P is activated by high intracellular concentrations of ATP and fructose 1, 6-bisphosphate, and a large portion of HPr is present as HPr(Ser-P) (Hanson et al., 2002; Jault et al., 2000; Ludwig et al., 2002; Monedero et al., 2001). This form of HPr forms a complex with the transcription factor CcpA, and the complex binds target sites (catabolite reponsive elements) in the promoter regions of genes that are subject to catabolite regulation. Binding of the $\mathrm{CcpA}-\mathrm{HPr}(\operatorname{Ser}-\mathrm{P})$ complex results in transcription repression or activation of large sets of genes and operons (Blencke et al., 2003; Moreno et al., 2001). If the preferred carbon sources become limiting, the activity of HPrK/P switches and the protein acts as a phosphorylase to dephosphorylate $\operatorname{HPr}(\operatorname{Ser}-\mathrm{P})$ (Kravanja et al., 1999; Mijakovic et al., 2002). This results in the dissociation of the CcpA-HPr(Ser-P) complex and thus in relief of carbon catabolite repression by CcpA.

In addition to the HPrK/P, B. subtilis encodes several other serine/threonine protein kinases and phosphatases, and many proteins are phosphorylated on serine or threonine residues in vivo (Leviné et al., 2006; Obuchowski, 2005). Three of the kinases and four of the phosphatases are involved in the control of sigma factor activities (Hughes and Mathee, 1998). For three other suspected serine/ threonine kinases it is not even clear whether they have a biological activity as kinases. Finally, the PrkC serine kinase phosphorylates the translation factor EF-G (Gaidenko et al., 2002). The largest group of the serine phosphatases found in B. subtilis belongs to the metallophosphatases of the PP2C family (Obuchowski, 2005). Among the PP2C phosphatases is the PrpC phosphatase which is encoded in an operon 
with the PrkC kinase and which was shown to dephosphorylate PrkC (if autophosphorylated on a threonine residue) as well as EF-G that had been phophorylated by PrkC (Gaidenko et al., 2002; Obuchowski et al., 2000). The prpC-prkC gene couple encoding the phosphatase and kinase is present in many bacteria among them the mollicutes such as Mycoplasma genitalium and Mycoplasma pneumoniae (Halbedel et al., 2006; Obuchowski et al., 2000). Recently, we provided evidence that the PrpC phosphatase of M. pneumoniae is capable of dephosphorylating HPr(Ser-P) and that this enzyme is involved in controlling the intracellular phosphorylation state of HPr (Halbedel et al., 2006).

In this work, we studied the biochemical activity of B. subtilis $\operatorname{PrpC}$ with $\operatorname{HPr}(\operatorname{Ser}-\mathrm{P})$ as the substrate. We provide evidence that $\mathrm{PrpC}$ is involved in preventing the accumulation of $\mathrm{HPr}($ Ser-P) in cells grown in the absence of glucose.

\section{Experimental procedures}

\section{Bacterial strains and growth conditions}

B. subtilis strain GP278 (trpC2 $\Delta x y l R:: e r m C$ amyE::xynP-lacZ) was used as wild type reference. This strain was obtained by transformation of B. subtilis QB7144 (Galinier et al., 1999) with plasmid pIW11xylR (Kraus et al., 1994). The prpC deletion strain GP281 was constructed by transformation of GP278 with chromosomal DNA of strain OMG401 (AprpC::aphA3) (Obuchowski et al., 2000). The E. coli strains BL21 (DE3) (Sambrook et al., 1989), NM522 (Gough and Murray, 1983) and DH5 $\alpha$ (Sambrook et al., 1989) were used as hosts for the overproduction of PrpC, HPr and HPrK/P, respectively. E. coli was grown in Luria-Bertani broth supplemented with $100 \mu \mathrm{g} / \mathrm{ml}$ ampicillin when necessary. For western blotting experiments B. subtilis strains were grown in C minimal medium (Faires et al., 1999) supplemented with L-tryptophan $(100 \mu \mathrm{g} / \mathrm{ml})$, potassium glutamate $(0.8 \%, \mathrm{~g} / \mathrm{l})$ and sodium succinate $(0.6 \%, \mathrm{~g} / \mathrm{l})$ as single carbon source.

\section{Protein purification}

Hexa-histidine tagged recombinant $\operatorname{PrpC}, \mathrm{HPr}$ and HPrK/P proteins were purified as described previously using the expression vectors pE5635, pAG2, and pGP205, respectively (Gaidenko et al., 2002; Galinier et al., 1997; Hanson et al., 2002). Briefly, the E. coli transformants were grown to an $\mathrm{OD}_{600}=0.8$ and protein expression was induced by the addition of $1 \mathrm{mM}$ IPTG. Growth was continued 3 hours before the cultures were harvested and passed through a French pressure cell at 1000 p.s.i. The lysates were centrifuged at 45000 
rpm for 45 min (Sorvall ultra pro, Dupont) and the supernatants were loaded on $\mathrm{Ni}^{+2}-\mathrm{NTA}$ superflow columns (Qiagen). Proteins were eluted with an imidazole gradient and fractions containing the pure proteins were pooled.

\section{Preparation of serine-phosphorylated HPr}

(His) ${ }_{6}-\mathrm{HPr}\left(\mathrm{Ser}-\mathrm{P}\right.$ ) was prepared as described (Hanson et al., 2002). Briefly, purified (His) $6^{-}$ $\mathrm{HPr}(20 \mu \mathrm{M})$ was mixed with $350 \mathrm{nM} \mathrm{HPrK} / \mathrm{P}$ protein and incubated at $37^{\circ} \mathrm{C}$ for $30 \mathrm{~min}$ in an assay buffer containing $25 \mathrm{mM}$ Tris/ $\mathrm{HCl} \mathrm{pH}$ 7.5, $10 \mathrm{mM} \mathrm{MgCl}, 1 \mathrm{mM}$ DTT, $10 \mathrm{mM}$ ATP and $20 \mathrm{mM}$ fructose-1,6-bisphosphate. The reaction was stopped by thermal inactivation of $\mathrm{HPrK} / \mathrm{P}$ at $95^{\circ} \mathrm{C}$ for $5 \mathrm{~min}$ and (His) ${ }_{6}-\mathrm{HPr}(\mathrm{Ser}-\mathrm{P})$ was purified by passing the reaction mixture through ultrafiltration spin columns (5 KDa exclusion size) according to the manufacturers protocol (Sartorius).

\section{Dephosphorylation of $\mathrm{HPr}(\mathrm{Ser}-\mathrm{P})$ and of para-nitrophenyl phosphate (PNPP) by PrpC in vitro}

For the dephosphorylation of (His) $)_{6}$-HPr(Ser-P) by PrpC, activity assays were carried out with varying amounts of purified PrpC in $50 \mu \mathrm{l}$ dephosphorylation assay buffer containing $50 \mathrm{mM}$ Tris/HCl pH 7.5, $2 \mathrm{mM} \mathrm{MnCl}_{2}, 1 \mathrm{mM}$ dithiothreitol (DTT) and $8 \mu \mathrm{g}$ (His) ( $_{6}$-HPr(Ser-P) at $37^{\circ} \mathrm{C}$ for $40 \mathrm{~min}$. The reactions were stopped by placing the samples on ice before they were loaded on $10 \%$ native polyacrylamide gels (Ludwig et al., 2002) followed by coomassie blue staining. Dephosphorylation assays with PNPP as a substrate were performed by adding 0.9 $\mu \mathrm{M}$ PrpC to $1 \mathrm{ml}$ dephosphorylation assay buffer containing $25 \mathrm{mM}$ PNPP instead of (His) $6^{-}$ $\mathrm{HPr}(\operatorname{Ser}-\mathrm{P})$. The reactions were incubated at $30^{\circ} \mathrm{C}$ for $25 \mathrm{~min}$ and stopped by the addition of $100 \mu \mathrm{l}$ of $0.5 \mathrm{M}$ EDTA $\mathrm{pH}$ 8.0. The end product $p$-nitrophenol was photometrically quantified by measuring the absorbance at $420 \mathrm{~nm}$.

\section{Western blotting}

The phosphorylation state of HPr in vivo was assayed by western blot analysis as follows: $B$. subtilis strains were grown to an $\mathrm{OD}_{600}$ of 0.6 and then $\mathrm{HCl}$ was added directly to the culture in order to abolish phosphatase activity of HPrK/P by decreasing the $\mathrm{pH}$ to 4.5 . Crude cell extracts were prepared as described (Gauthier et al., 1997) and $1 \mu \mathrm{g}$ of total cell protein was loaded on $10 \%$ native polyacrylamide gels, allowing the separation of phosphorylated and non-phosphorylated HPr. To distinguish HPr(His-P) from $\mathrm{HPr}(\mathrm{Ser}-\mathrm{P})$, a second aliquot of each crude extract was incubated at $70^{\circ} \mathrm{C}$ for $10 \mathrm{~min}$ before gel electrophoresis. Proteins were 
blotted to a PVDF membrane and HPr species were detected using polyclonal rabbit antibodies directed against B. subtilis HPr (Monedero et al., 2001).

\section{Results and Discussion}

\section{HPr(Ser-P) is a target for PrpC}

In a previous work, we have shown that the PrpC phosphatase of $M$. pneumoniae can dephosphorylate HPr(Ser-P) (Halbedel et al., 2006). We considered therefore the possibility that the B. subtilis phosphatase might also be capable of using $\operatorname{HPr}(\operatorname{Ser}-\mathrm{P})$ as a substrate. To test this idea, we purified the His-tagged B. subtilis HPr and HPrK/P and phosphorylated HPr at Ser-46 on a preparative scale. Moreover, the B. subtilis $\operatorname{PrpC}$ phosphatase was purified. The purified $\operatorname{HPr}(\operatorname{Ser}-\mathrm{P})$ was used in a dephosphorylation assay to detect PrpC phosphatase activity. As shown in Fig. 5, HPr(Ser-P) migrates much faster than non-phosphorylated HPr on a native polyacrylamide gel. The addition of $\operatorname{PrpC}$ resulted in a disappearance of the phosphorylated form of HPr whereas we observed an accumulation of non-phosphorylated HPr. As can be seen in Fig. 5, small amounts of PrpC were sufficient to cause complete dephosphorylation. These findings indicate that $\operatorname{PrpC}$ is capable of using $\operatorname{HPr}(\operatorname{Ser}-\mathrm{P})$ as a substrate in vitro.

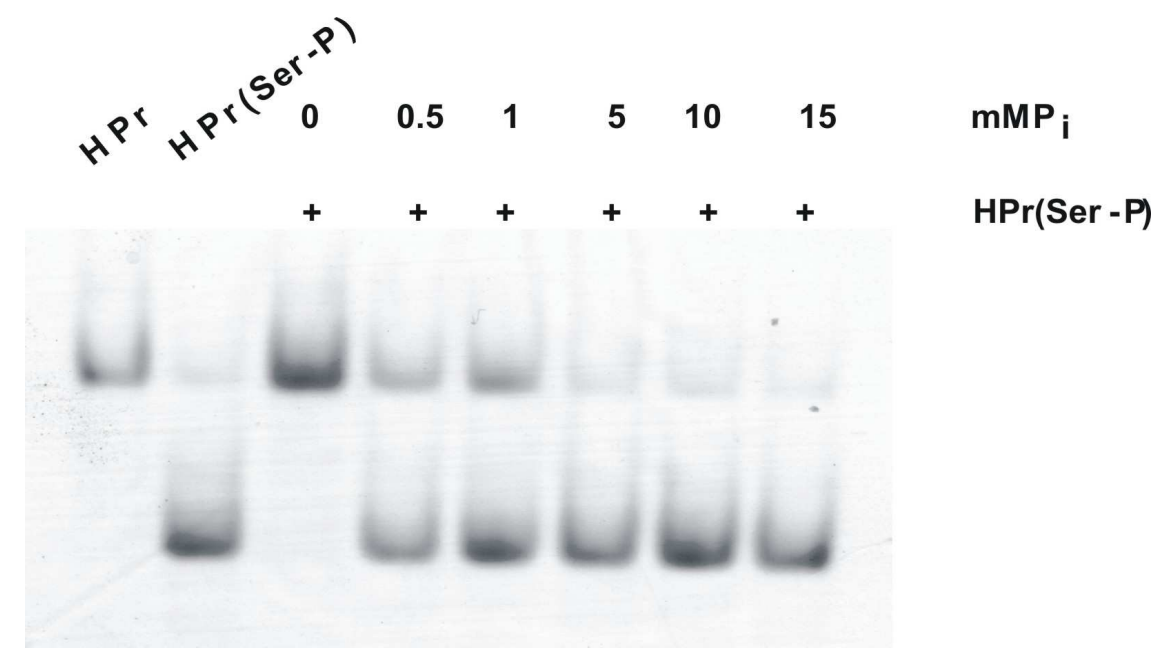

Figure. 5. Dephosphorylation of $\mathrm{HPr}(\operatorname{Ser}-\mathrm{P})$ by $\operatorname{PrpC}$ in vitro. Increasing amounts of purified PrpC were incubated with $16 \mu \mathrm{M}$ of $\mathrm{HPr}\left(\mathrm{Ser}-\mathrm{P}\right.$ in dephosphorylation assay buffer at $37^{\circ} \mathrm{C}$ for $40 \mathrm{~min}$ and phosphorylated and non-phosphorylated forms of HPr were separated on a $10 \%$ native polyacrylamide gel (lanes 3-8). $8 \mu \mathrm{g}$ of purified $\mathrm{HPr}$ and $\mathrm{HPr}(\mathrm{Ser}-\mathrm{P})$ were loaded in lane 1 and lane 2 respectively as controls. 


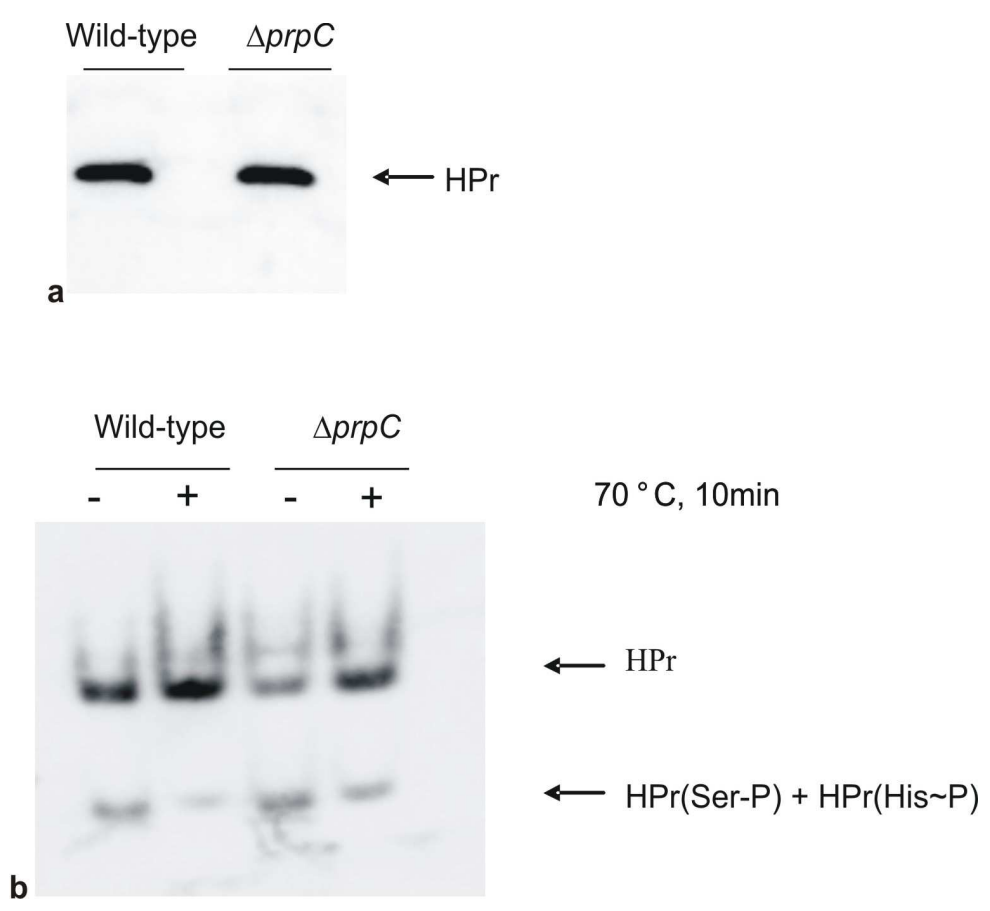

Figure. 6. Deletion of $p r p C$ increases the amount of $\mathrm{HPr}(\operatorname{Ser}-\mathrm{P})$ in vivo. The wild type strain GP278 and the $\operatorname{prpC}$ mutant GP281 were grown in minimal medium supplemented with succinate as a single carbon source. a Comparison of the amounts of cellular HPr in the wild type strain and the prpC mutant. Total cell protein $(5 \mu \mathrm{g})$ of the wild type strain GP278 and the $\operatorname{prpC}$ mutant GP281 was loaded onto $15 \%$ denaturing SDS polyacrylamide gel followed by elctroblotting onto PVDF membrane. $\mathrm{HPr}$ was then detected using polyclonal rabbit antiserum against B. subtilis HPr. b Determination of the HPr phosphorylation pattern. Protein extracts were separated on a $10 \%$ native polyacrylamide gel and HPr was detected by western blotting. Prior to loading, an aliquot of each cell extract was incubated at $70^{\circ} \mathrm{C}$ for $10 \mathrm{~min}$ to cause the loss of the histidine-bonded phosphoryl-group leaving $\mathrm{HPr}(\mathrm{Ser}-\mathrm{P})$ as single phosphorylated form (lanes 2 and 4 ).

\section{PrpC affects the HPr phosphorylation state in vivo}

In B. subtilis growing in glucose, a large part of HPr is phosphorylated on Ser-46 by HPrK/P. In contrast, only a small fraction of $\operatorname{HPr}(\operatorname{Ser}-\mathrm{P})$ is present in minimal medium without glucose, due to the low kinase and predominant phosphorylase activities of HPrK/P under these conditions (Ludwig et al., 2002; Monedero et al., 2001). Since PrpC is capable of dephosphorylating $\operatorname{HPr}(\mathrm{Ser}-\mathrm{P})$ in vitro we asked whether it might also be involved in controlling the HPr phosphorylation state in vivo and thus contribute to the low amount of $\mathrm{HPr}(\mathrm{Ser}-\mathrm{P})$ observed in glucose-free minimal medium. To address this question, we grew the B. subtilis wild type strain GP278 and its isogenic prpC mutant GP281 in CSE minimal medium and prepared protein extracts for the analysis of the HPr amounts and the in vivo HPr phosphorylation pattern. The results of this experiment are shown in Fig. 6. In both strains, comparable amounts of $\mathrm{HPr}$ were present (Fig. 6a). Both phosphorylated and nonphosphorylated HPr were detected in the wild type strain (Fig. 6b). 
The molecular nature of the phosphorylation can easily be revealed by heating the cell extracts: histidyl-phosphate is heat-labile, whereas seryl-phosphate is heat-stable. We observed that the phosphorylation of HPr disappeared upon heating of an aliquot of the cell extract. This indicates that HPr in the wild type strain had been phosphorylated predominantly on His-15. This finding is in good agreement with previous studies (Ludwig et al., 2002; Monedero et al., 2001). A different result was obtained with the isogenic prpC mutant GP281. In this strain, a larger portion of HPr was present in the phosphorylated form (Fig. 6b). Upon heating, a small but significant fraction of heat-stable phosphorylation was detected demonstrating that $\mathrm{HPr}(\mathrm{Ser}-\mathrm{P})$ is present in this mutant even in glucose-free CSE medium. A quantitative analysis revealed that about $10 \%$ of HPr are phosphorylated on Ser-46 in the wild type strain whereas $30 \%$ of $\operatorname{HPr}(\operatorname{Ser}-\mathrm{P})$ were detected in the prpC mutant GP281. Thus, PrpC is involved in the control of the in vivo HPr phosphorylation state and is required for complete dephosphorylation of HPr in cells grown in the absence of glucose.

\section{Inhibition of PrpC activity by inorganic phosphate}

Previously, the B. subtilis PrpC activity was shown to be inhibited by $\beta$-glycerolphosphate and some divalent cations such as $\mathrm{Ca}^{2+}$ and $\mathrm{Zn}^{2+}$ (Obuchowski et al., 2000). In M. pneumoniae, PrpC is also subject to inhibition by inorganic phosphate (Halbedel et al., 2006). We tested therefore the response of $B$. subtilis PrpC activity to the presence of increasing concentrations of inorganic phosphate $\left(\mathrm{P}_{\mathrm{i}}\right)$. As shown in Fig. 8, complete dephosphorylation of $\operatorname{HPr}($ Ser-P) was observed in the absence of any phosphate. In contrast, the presence of 0.5 $m M P_{i}$ was sufficient to cause a partial inhibition of PrpC activity, and this inhibition was even more pronounced at higher $\mathrm{P}_{\mathrm{i}}$ concentrations.

To get a quantitative impression of the inhibition of PrpC by $\mathrm{P}_{\mathrm{i}}$, we studied the activity of PrpC using the synthetic substrate $p$-nitrophenyl phosphate (PNPP). The $K_{M}$ and $v_{\max }$ values have been reported previously (Obuchowski et al., 2000). In the presence of $P_{i}$, the PrpC activity was strongly inhibited in a competitive manner $\left(\mathrm{K}_{\mathrm{i}} 110 \pm 10 \mu \mathrm{M}\right)$ (Fig. 7). As found for the other kinetic parameters (Halbedel et al., 2006; Obuchowski et al., 2000), the enzymes from $M$. pneumoniae and B. subtilis are similar also with respect to inhibition by phosphate.

It is interesting to note that the intracellular concentration of $\mathrm{P}_{\mathrm{i}}$ is high under the conditions that were used to observe PrpC-dependent HPr(Ser-P) dephosphorylation (Mijakovic et al., 2002). The results presented in Fig. 6b suggest that the inhibitory effect of $\mathrm{P}_{\mathrm{i}}$ observed in vitro is counteracted in vivo, possibly by other metabolites. This idea is in good 
agreement with our observation that PrpC is only a minor player in the control of the HPr phosphorylation state (see Fig. 6b).

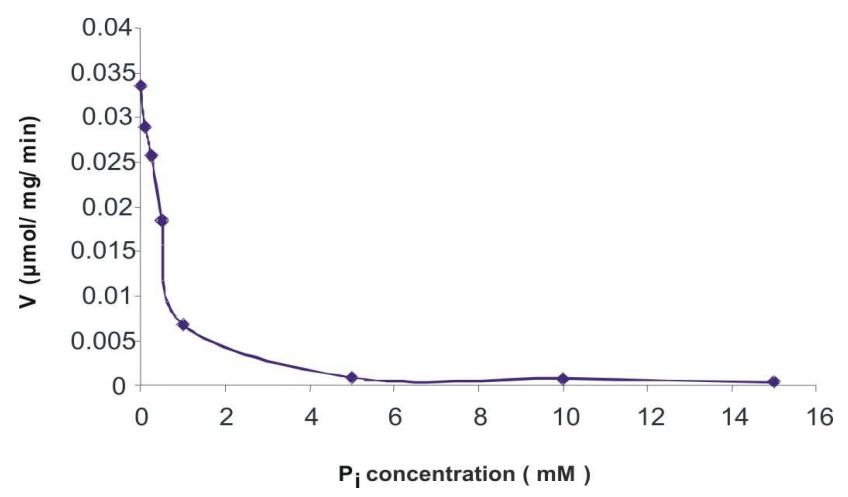

Figure. 7. Inhibition of PrpC phosphatase activity towards PNPP by inorganic phosphate. Dephosphorylation of para-nitrophenyl phosphate (PNPP) by $\operatorname{PrpC}(0.9 \mu \mathrm{M})$ in the presence of increasing concentrations of inorganic phosphate $[0.5-15 \mathrm{mM}]$ was investigated. The rate of para-nitrophenol formation is plotted against the $\mathrm{P}_{\mathrm{i}}$ concentration.

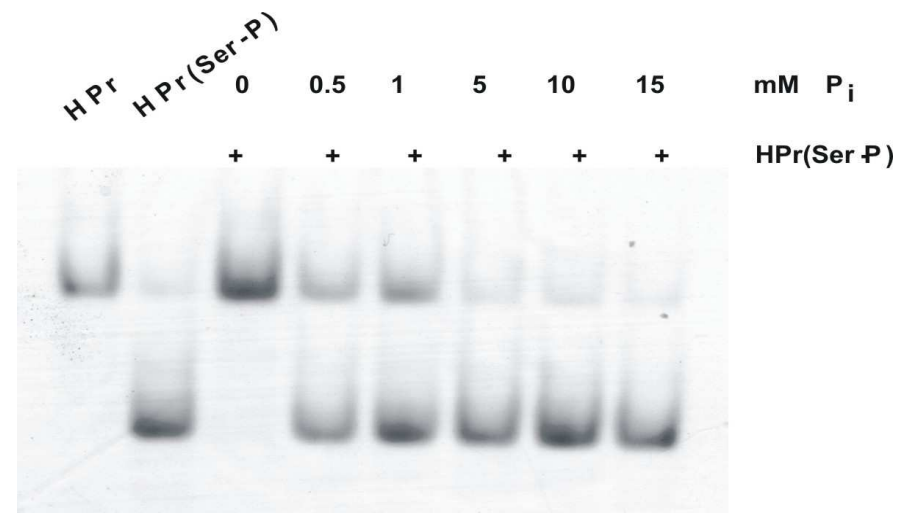

Figure. 8. Inhibition of PrpC phosphatase activity towards its natural target $\operatorname{HPr}(\operatorname{Ser}-\mathrm{P})$ by inorganic phosphate. Dephosphorylation of $\operatorname{HPr}(\operatorname{Ser}-\mathrm{P})$ by $\operatorname{PrpC}(0.9 \mu \mathrm{M})$ was studied in the presence of different $P_{i}$ concentrations (lanes 3-8). Subsequently, phosphorylated and nonphosphorylated forms of HPr were separated on a $10 \%$ native polyacrylamide gel. Purified HPr and $\mathrm{HPr}($ Ser-P) $(5 \mu \mathrm{g})$ were loaded as controls in lanes 1 and 2, respectively.

\section{PrpC-catalyzed HPr(Ser-P) dephosphorylation in search of a function}

As shown in this work, PrpC contributes in keeping the cellular level of $\operatorname{HPr}(\operatorname{Ser}-\mathrm{P})$ low in a glucose-free medium. This might ensure that no $\mathrm{HPr}(\operatorname{Ser}-\mathrm{P})$ is available for $\mathrm{CcpA}$-dependent gene regulation in the absence of glucose. If this would be true, one would expect to detect some "constitutive repression" of genes subject to carbon catabolite repression in a glucosefree medium in a $\operatorname{prpC}$ mutant strain. To test this assumption we used the promoter of the $x y n P B$ operon encoding $\beta$-xyloside permease and $\beta$-xylosidase, which is about hundred-fold repressed in the presence of glucose (Galinier et al., 1999). However, the expression levels in 
glucose-free medium were similar for the wild type and the $\operatorname{prpC}$ mutant (data not shown) suggesting that PrpC is not involved in carbon catabolite repression. This seems to be surprising, but one has to keep in mind that only low amounts of $\operatorname{HPr}(\operatorname{Ser}-\mathrm{P})$ are present in the absence of PrpC. This concentration may not be sufficient to form a stable complex with CcpA. Moreover, the formation of the HPr(Ser-P)-CcpA complex and binding of the complex to the DNA target sites is simulated by fructose-1.6-bisphosphate (Seidel et al., 2005). However, the intracellular concentration of this metabolite is low in cells grown in the absence of glucose (Mijakovic et al., 2002). Thus, no CcpA-dependent catabolite repression might be possible even though low amounts of $\mathrm{HPr}(\mathrm{Ser}-\mathrm{P})$ are detectable in the $\operatorname{prpC}$ mutant.

The formation of $\mathrm{HPr}(\mathrm{Ser}-\mathrm{P})$ has a second effect which is not directly related to its activity as a cofactor in carbon catabolite repression: $\mathrm{HPr}(\mathrm{Ser}-\mathrm{P})$ is a very poor target for Enzyme I of the PTS and is therefore not available for sugar transport (Deutscher et al., 1984). This results in impaired PTS sugar transport if a large portion of HPr is phosphorylated on Ser-46 (Ludwig et al., 2002). Thus, a complete dephosphorylation of HPr jointly achieved by the activities of HPrK/P and PrpC may ensure the availability of free HPr for PTS phosphate transfer to all sugar permeases and may thus be a precaution allowing a rapid adaptation to an improving nutrient supply.

\section{Conclusion}

Our data suggest that PrpC is capable of dephosphorylating $\operatorname{HPr}(\operatorname{Ser}-\mathrm{P})$. However, this activity seems to be of limited relevance in living cells of B. subtilis, and $\mathrm{HPr}(\operatorname{Ser}-\mathrm{P})$ may be just a minor substrate for PrpC in vivo. While the physiological role of PrpC-dependent $\mathrm{HPr}($ Ser-P) dephosphorylation in B. subtilis remains to be uncovered, it may be important in organisms in which the HPrK/P does not exhibit phosphorylase activity, as shown for $M$. pneumoniae (Halbedel et al., 2006). With the discovery of HPrK/P in many bacteria, among them proteobacteria and spirochaetes, PrpC is an attractive candidate to study the control of the HPr phosphorylation state in those bacteria. 


\section{Carbon catabolite repression in Bacillus subtilis: A quantitative analysis of repression exerted by different carbon sources}

The results described in this chapter were published in:

Singh,K.D., Schmalisch,M.H., Stülke,J., and Görke,B. (2008) Carbon Catabolite Repression in Bacillus subtilis: A Quantitative Analysis of Repression Exerted by Different Carbon Sources. J Bacteriol. DOI: 10.1128/JB.00848-08

\section{Author contributions:}

This study was designed by Kalpana Singh, Jörg Stülke and Boris Görke. All experiments were performed by Kalpana Singh. Matthias Schmalisch performed the experiment for the estimation of intracellular FBP levels and started this project during his Ph.D. work. This paper was written by Boris Görke. 


\begin{abstract}
In many bacteria glucose is the preferred carbon source and represses the utilization of secondary substrates. In Bacillus subtilis, this carbon catabolite repression (CCR) is achieved by the global transcription regulator CcpA, whose activity is triggered by the availability of its phosphorylated cofactors $\mathrm{HPr}(\operatorname{Ser} 46-\mathrm{P})$ and $\mathrm{Crh}(\operatorname{Ser} 46-\mathrm{P})$. Phosphorylation of these proteins is catalyzed by the metabolite-controlled HPr kinase HPrK/P. Recent studies focused on glucose as repressing substrate. Here, we show that many carbohydrates cause CCR. The substrates form a hierarchy in their ability to exert repression via the CcpA-mediated CCR pathway. Of the two co-factors, HPr is sufficient for complete CCR. In contrast, Crh cannot substitute for HPr on substrates that cause a strong repression. Determination of the phosphorylation state of HPr in vivo revealed a correlation between the strength of repression and the degree of phosphorylation of HPr at Ser-46. Sugars transported by the phosphotransferase system (PTS) cause the strongest repression. However, the phosphorylation state of HPr at its His-15 residue and PTS transport activity have no impact on the global CCR mechanism, which is a major difference to the mechanism operative in Escherichia coli. Our data suggest that the hierarchy in CCR exerted by the different substrates is exclusively determined by the activity of HPrK/P.
\end{abstract}

\title{
Introduction
}

As for any organism, nutrient supply is of prime importance for bacteria. In their natural habitats, bacteria often encounter a mixture of different carbon sources that can potentially be used. Therefore, mechanisms have evolved in many bacteria that enable the selective uptake and metabolism of those carbon sources that allow the most rapid growth and that promise the best success in the competition with other bacteria or fungi. For many heterotrophic bacteria, glucose is the preferred source of carbon. In the presence of glucose, the genes required for the utilization of secondary carbon sources are not expressed and pre-existing enzymes are often inactivated to prevent the waste of resources. This phenomenon is referred to as carbon catabolite repression (CCR, for reviews see (Deutscher, 2008; Görke and Stülke, 2008).

CCR has been extensively studied in the model bacteria Bacillus subtilis and Escherichia coli. Although the physiological outcome is very similar, the global mechanisms underlying CCR are completely different in these bacteria. In E. coli and other enteric bacteria, the EIIA ${ }^{\mathrm{Glu}}$ domain of the glucose transporter is the central processing unit in CCR. 
This protein is part of the phosphoenolpyruvate (PEP):carbohydrate phosphotransferase system (PTS), which is responsible for the uptake and concomitant phosphorylation of numerous carbohydrates in many bacteria (Deutscher et al., 2006). In this system, the two general phosphotransferases, Enzyme I (EI) and histidine protein (HPr), transfer phosphorylgroups from PEP to the various sugar transporters, named Enzymes II (EIIs). In the absence of glucose, the EIIA ${ }^{\text {Glu }}$ domain is preferentially phosphorylated. In this form, EIIA ${ }^{\text {Glc }}$ activates the adenylate cyclase, which leads to an increase in the intracellular cAMP concentration. Binding of cAMP activates the transcription activator CRP (cAMP receptor protein), which is in turn required for the expression of numerous secondary catabolic genes. In the presence of glucose, EIIA ${ }^{\text {Glu }}$ is predominantly un-phosphorylated and therefore unable to activate adenylate cyclase. In addition, un-phosphorylated EIIA ${ }^{\text {Glu }}$ inhibits transporters of secondary carbon sources by direct interaction. This operon-specific mechanism, which has been termed inducer exclusion, contributes to the repression of catabolic genes in the presence of glucose. In some cases, e.g. the E. coli lac operon, inducer exclusion might even be the decisive mechanism for CCR (Görke and Stülke, 2008).

A different mechanism of CCR is operative in the Gram-positive soil bacterium $B$. subtilis and other Firmicutes. Here, the global mechanism of CCR is mediated by the pleiotropic transcription factor CcpA (for reviews see (Fujita et al., 2007; Warner and Lolkema, 2003). In the presence of glucose, CcpA represses several hundred catabolic genes and activates the transcription of some genes of overflow metabolism (Blencke et al., 2003; Lulko et al., 2007; Moreno et al., 2001; Yoshida et al., 2001). The ability of CcpA to bind its target sites, the catabolite responsive elements (cre), is in turn controlled by the presence of its cofactors, $\operatorname{HPr}(\operatorname{Ser}-\mathrm{P})$ and $\operatorname{Crh}(\operatorname{Ser}-\mathrm{P})$ (Galinier et al., 1999; Schumacher et al., 2004; Schumacher et al., 2006). Unlike HPr in E. coli, the B. subtilis homolog contains a regulatory phosphorylation site, Ser-46, in addition to His-15, which is phosphorylated during phosphate transfer to the transported sugar. Crh is homologous to HPr, but it lacks His-15 and is therefore unable to participate in sugar transport (Galinier et al., 1997). In the presence of glucose, HPr, and presumably also Crh, are phosphorylated by the bi-functional HPr kinase/ phosphorylase (HPrK/P) on Ser-46, whereas this site is less phosphorylated in cells growing in the absence of sugars (Ludwig et al., 2002; Monedero et al., 2001). Dephosphorylation of $\mathrm{HPr}(\operatorname{Ser}-\mathrm{P})$ is catalyzed by the phosphorylase activity of HPrK/P (Mijakovic et al., 2002) and, to some extent, by the protein phosphatase PrpC (Singh et al., 2007). In vitro experiments suggested that the two antagonistic activities of $\mathrm{HPrK} / \mathrm{P}$ are regulated by metabolites. High fructose 1,6-bisphosphate (FBP) and ATP or $\mathrm{PP}_{\mathrm{i}}$ concentrations stimulate the kinase activity, 
whereas the phosphorylase activity prevails when the $\mathrm{P}_{\mathrm{i}}$ concentration is high (Jault et al., 2000; Ramström et al., 2003; Reizer et al., 1998). Only HPr(Ser-P), but none of the other HPr forms, is able to productively interact with CcpA and to exert CCR (Reizer et al., 1996; Schumacher et al., 2004). In vitro experiments suggested that in addition to its role in the activation of HPrK kinase, FBP enhances DNA binding of the CcpA/HPr(Ser-P) complex by directly binding to CcpA (Schumacher et al., 2007; Seidel et al., 2005). The role of Crh in CCR is still unclear.

Traditionally, studies dealing with CCR have been focused on the repression exerted by glucose. Therefore, the terms carbon catabolite repression and glucose repression are often used synonymously. However, for the Gram-negative bacterium E. coli it was shown that a large number of carbohydrates in addition to glucose exert CCR (Bettenbrock et al., 2007; Hogema et al., 1998). Much less is known in B. subtilis. So far, CCR exerted by carbon sources different from glucose has not been systematically studied. In this work, we analyzed CcpA-mediated CCR exerted by a variety of carbon sources in B. subtilis. As a model for this study, we chose the $x y n P B$ operon, which encodes a $\beta$-xyloside transporter and $\beta$-xylosidase. These functions allow the uptake and degradation of $\beta$-xylosides to xylose (Fig. 9), (Galinier et al., 1999; Lindner et al., 1994). Xylose is subsequently converted to xylulose-5-P by enzymes encoded in the $x y l A B$ operon located downstream of the $x y n P B$ operon (Fig. 9). Both operons are repressed by the XylR protein, which binds to operator sites present in the respective promoter regions and prevents transcription initiation (Dahl et al., 1994; Galinier et $a l .$, 1999). Binding of xylose releases XylR from its operator sites. B. subtilis is not able to grow on xylose due to the lack of a xylose-specific permease (Lindner et al., 1994; Schmiedel and Hillen, 1996a; Schmiedel and Hillen, 1996b). However, xylose can be slowly taken up by the AraE protein, which is sufficient for induction of $x y n P B$ and $x y l A B$ expression (Krispin and Allmansberger, 1998). In the presence of glucose, both operons are strongly repressed. Downstream of their promoters, cre sites are present, which are bound by the HPr-Ser46P/CcpA complex thereby preventing transcription initiation (Galinier et al., 1999; Kraus et al., 1994).

In this study, we show that a variety of carbohydrates represses $x y n P B$ transcription via the CcpA-mediated CCR pathway, but each to a different degree. In general, most substrates transported by the PTS caused a strong repression, whereas CCR by non-PTS substrates was weaker. The Crh protein was completely dispensable for repression exerted by all of these substrates, whereas HPr was essential for the strong CCR caused by sugars like glucose, fructose and mannitol. Analysis of the HPr phosphorylation state in vivo revealed 
that the strength of repression exerted by a particular substrate correlates well with the amount of $\operatorname{HPr}(\operatorname{Ser}-\mathrm{P})$ in the cell. However, in contrast to E. coli, transport activity of the PTS has no direct role for the global CCR mechanism. Our data suggest that in B. subtilis the strength of CcpA-mediated CCR is determined exclusively by the metabolite-controlled activity of $\mathrm{HPrK} / \mathrm{P}$.

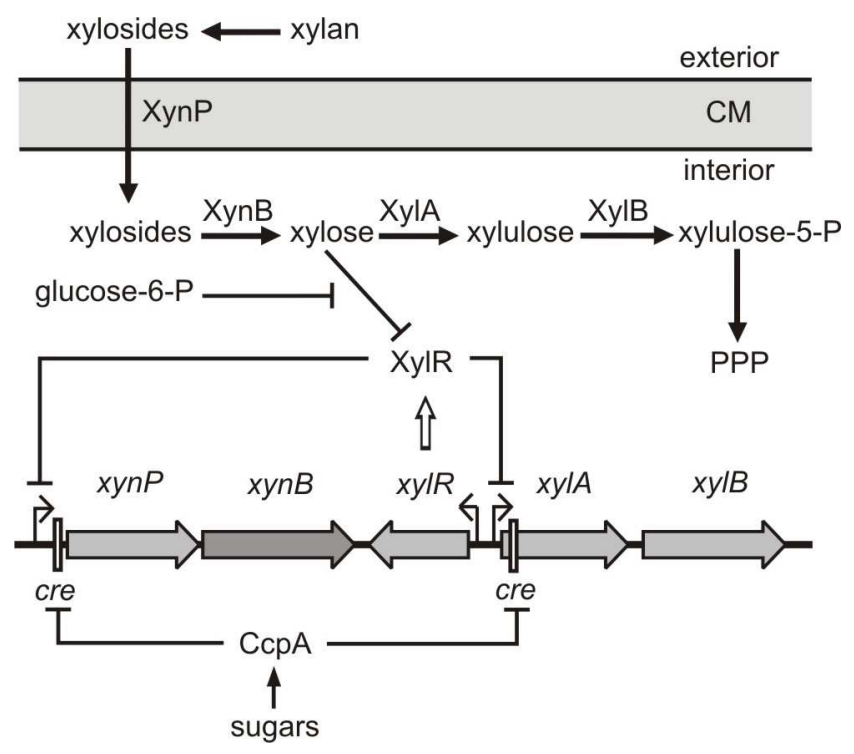

Figure. 9. Utilization of $\boldsymbol{\beta}$-xylan in $\boldsymbol{B}$. subtilis. Xylan can be degraded by an extracellular xylanase to $\beta$-xylosides, which are subsequently taken up by the $\beta$-xyloside transporter XynP encoded in the bi-cistronic $x y n P B$ operon. The $\beta$-xylosidase, $\mathrm{XynB}$ converts $\beta$-xylosides to xylose, which is further converted to xylulose-5-P by the functions encoded in the adjacent $x y l A B$ operon. Xylulose-5-P finally enters the pentose phosphate pathway (PPP). The $x y n P B$ and $x y l A B$ operons are repressed by binding of the Xyl repressor (XylR) to operator sites in the absence of the inducer xylose. Both operons are subject to global CCR, which is mediated by binding of CcpA to cre sites downstream of the promoters. In addition, glucose-6-phosphate contributes to CCR by acting as an anti-inducer for XylR.

\section{Materials and Methods}

\section{Bacterial strains and growth conditions}

The B. subtilis strains used in this study are listed in Table 2. The presence of the ptsH1 mutation was verified by sequencing of chromosomal DNA of the relevant strains. E. coli DH5 $\alpha$ (Sambrook and Russell, 2001) was used for plasmid propagation. B. subtilis was grown in CSE minimal medium supplemented with auxotrophic requirements (at $50 \mathrm{mg} \mathrm{l}^{-1}$ ) (Martin-Verstraete et al., 1995). If necessary, xylose was added at a concentration of $0.2 \%$ $(\mathrm{w} / \mathrm{v})$. Potentially repressing carbon sources were used at a concentration of $0.5 \%(\mathrm{w} / \mathrm{v})$. $E$. coli was grown in LB medium and transformants were selected on plates containing ampicillin $\left(100 \mu \mathrm{g} \mathrm{ml}^{-1}\right)$. 
LB and SP plates were prepared by the addition of $17 \mathrm{~g}^{-1}$ Bacto agar (Difco) to the medium. For the determination of growth rates, pre-cultures were grown overnight in CE minimal medium supplemented with auxotrophic requirements (at $50 \mathrm{mg} \mathrm{l}^{-1}$ ) and a single carbon source at a concentration of $0.5 \%(\mathrm{w} / \mathrm{v})$. The cells were inoculated in the same medium to an $\mathrm{OD}_{600}$ of 0.1 and the bacteria were incubated at $37^{\circ} \mathrm{C}$ at 200 r.p.m. and the turbidity at $600 \mathrm{~nm}$ was recorded periodically. The growth rates $(\mu)$ were determined from the exponential phases of the growth curves using the formula $\mu=\operatorname{logb}-\log B /(\log 2) \times(\Delta t)$, where $\Delta t$ is the time interval in min, $\mathrm{b}$ the $\mathrm{OD}_{600}$ of the culture at the end, and $\mathrm{B}$ the $\mathrm{OD}_{600}$ at the beginning of this time interval.

\section{DNA manipulation}

Transformation of E. coli and plasmid DNA extraction was performed using standard procedures (Sambrook and Russell, 2001). Restriction enzymes and DNA polymerases were used as recommended by the manufacturers. Chromosomal DNA of B. subtilis was isolated using the DNeasy Tissue Kit (Quiagen) according to the supplier's protocol. Plasmid pIW11xylR used for inactivation of the $x y l R$ gene has been described previously (Kraus et al., 1994). Plasmid pGP811 used for the disruption of ptsI is a derivative of plasmid pHT181 (Lereclus and Arantes, 1992) carrying the $591 \mathrm{bp}$ EcoRI fragment of ptsI inserted in its unique EcoRI site. Plasmid pGP650 carries the $h p r K-G 158 \mathrm{~A}$ allele under control of the constitutive $\operatorname{deg} Q 36$ promoter. The $h p r K$ allele was amplified using primers SK11 (GGCGGATCCGTGGCAAAGGTTCGCACAAAAGA) KS12 (AAAAAGCTTGGTTCTATCGCTTCATTCATTTAACGC) and plasmid pGP407 (Hanson et al., 2002) as template. Subsequently, the PCR fragment was digested with BamHI and HindIII and inserted between the same sites of plasmid pGP380 (Herzberg et al., 2007).

\section{Transformation and enzyme assays}

B. subtilis was transformed with plasmids and chromosomal DNA according to the two-step protocol (Kunst and Rapoport, 1995). Transformants were selected on SP plates containing spectinomycin $\left(100 \mu \mathrm{g} \mathrm{ml}^{-1}\right)$, kanamycin $\left(5 \mu \mathrm{g} \mathrm{ml}^{-1}\right)$, or erythromycin plus lincomycin $(2$ and $25 \mu \mathrm{g} \mathrm{ml}{ }^{-1}$, respectively). For enzyme assays cells were harvested in exponential growth phase at an $\mathrm{OD}_{600}$ of $0.6-0.8$. $\beta$-Galactosidase and $\beta$-xylosidase activities were determined in cell extracts using $o$-nitrophenyl galactopyranoside and $p$-nitrophenyl xyloside as substrates, respectively (Lindner et al., 1994; Sambrook and Russell, 2001). 


\section{Western blot analysis}

For Western blot analyses, crude cell extracts were separated by SDS PAGE and transferred to a polyvinylidene difluoride membrane (PVDF, BioRad) by electroblotting. The proteins were detected with rabbit polyclonal antisera raised against CcpA, HPr, or HPrK/P of Bacillus megaterium or B. subtilis (Hanson et al., 2002; Küster et al., 1996; Monedero et al., 2001). The antibodies were visualized by using anti-rabbit IgG-AP secondary antibodies (Chemikon International, Temecula, U.S.A.) and the CDP* detection system (Roche Diagnostics).

\section{Analysis of the phosphorylation state of HPr in vivo}

HPr phosphorylation was assayed in vivo by Western blot analysis as follows. Bacteria were grown in CSE in the presence of the indicated carbon sources to an $\mathrm{OD}_{600}$ of 0.6. Cells were disrupted using a French press, and crude extracts were prepared as described previously (Ludwig et al., 2002). Proteins (2 $\mu \mathrm{g}$, respectively) were separated on non-denaturing 12\% polyacrylamide gels. On these gels, phosphorylated HPr migrates faster than the nonphosphorylated protein. HPr(His-P) was dephosphorylated by incubation of the crude extract for $10 \mathrm{~min}$ at $70^{\circ} \mathrm{C}$. After electrophoresis, the proteins were blotted to a PVDF membrane. The different forms of HPr were detected using antibodies directed against B. subtilis HPr (Monedero et al., 2001).

\section{Determination of fructose 1,6-bisphosphate concentrations}

Protein-free cell extracts for the determination of FBP concentrations in B. subtilis were prepared as described previously with few modifications (Mijakovic et al., 2002). Briefly, cells of the B. subtilis wild type strain 168 were grown in $50 \mathrm{ml} \mathrm{CSE} \mathrm{medium} \mathrm{in} \mathrm{the} \mathrm{presence}$ of the indicated carbon sources. For each growth condition at least three independent experiments were carried out. Cultures were harvested by centrifugation at room temperature for $5 \mathrm{~min}$ at 10,000g and pellets were subsequently frozen in liquid nitrogen. The pellets were resuspended in $0.6 \mathrm{M}$ of cold perchloric acid and subsequently incubated on ice for $20 \mathrm{~min}$. The precipitated proteins and cell debris were removed by centrifugation $\left(4^{\circ} \mathrm{C}, 5 \mathrm{~min}, 13,000\right.$ $\mathrm{rpm})$. The $\mathrm{pH}$ in the supernatant was adjusted to 7.4 with a solution of cold $0.6 \mathrm{M} \mathrm{KOH}$ in $100 \mathrm{mM}$ Tris- $\mathrm{HCl}$ ( $\mathrm{pH}$ 7.4). The precipitated $\mathrm{KClO}_{4}$ was removed by centrifugation. The FBP concentrations were determined in the supernatants as described previously (Mijakovic et al., 2002). 
Table. 2. B. subtilis strains used in this study.

\begin{tabular}{|c|c|c|}
\hline Strain & Genotype & Sourcea \\
\hline 168 & $\operatorname{trpC2}$ & Laboratory stock \\
\hline GP270 & $\operatorname{trpC} 2$ xylR::ermC & pIW11xylR $\rightarrow 168$ \\
\hline GP278 & trpC2 xylR::ermC amyE::(xynP-lacZ cat) & Singh et al., 2007 \\
\hline GP279 & $\begin{array}{l}\text { trpC2 xylR::ermC crh::spc amyE::(xynP-lacZ } \\
\text { cat) }\end{array}$ & QB7097 $\rightarrow$ GP278 \\
\hline GP284 & $\operatorname{trpC} 2$ xylR::ermC ptsH1 & pIW11xylR $\rightarrow$ QB5223 \\
\hline GP287 & $\operatorname{trpC} 2$ xylR::ermC ptsH1 crh::spc & pIW11xylR $\rightarrow$ QB7101 \\
\hline GP289 & $\operatorname{trpC} 2$ xylR::ermC hprK::aphA3 & $\mathrm{QB} 7160 \rightarrow \mathrm{GP} 270$ \\
\hline GP297 & $\operatorname{trpC} 2$ xylR::ermC crh::spc & QB7097 $\rightarrow$ GP270 \\
\hline GP853 & $\operatorname{trp} C 2$ xylR::ermC cсpA::spc & QB5407 $\rightarrow$ GP270 \\
\hline GP858 & $\operatorname{trpC} 2 \Delta h p r K:: a p h A 3$ & QB7160 $\rightarrow 168$ \\
\hline GP864 & $\operatorname{trpC} 2$ AptsI::ermC & pGP811 $\rightarrow 168$ \\
\hline QB5223 & $\operatorname{trpC} 2 \mathrm{ptsH1}$ & $\begin{array}{l}\text { Martin-Verstraete et al., } \\
1995\end{array}$ \\
\hline QB5407 & $\operatorname{trp} C 2$ сcpA::spc & Faires et al., 1999 \\
\hline QB7097 & $\operatorname{trpC} 2$ crh::spc & I. Martin-Verstraete \\
\hline QB7101 & trpC2 ptsH1 crh::spc & I. Martin-Verstraete \\
\hline QB7144 & $\operatorname{trpC} 2$ amyE::(xynP-lacZ cat) & Galinier et al., 1999 \\
\hline QB7160 & trpC2 amyE::(levD-lacZ cat) hprK::aphA3 & $\begin{array}{l}\text { Martin-Verstraete et al., } \\
1999\end{array}$ \\
\hline
\end{tabular}

${ }^{\mathrm{a}}$ Arrows indicate construction by transformation.

\section{Results}

\section{CCR of $\beta$-xylosidase exerted by different carbon sources.}

CCR has mainly been studied using glucose as repressing carbon source. To explore whether other carbon sources also exert CCR, we chose the $x y n P B$ operon as a model system. For this purpose, we measured the activity of the $\beta$-xylosidase $\mathrm{XynB}$ as a convenient reporter for regulatory events taking place at the $x y n P B$ promoter (see below). First, we tested the wildtype strain 168 in CSE minimal medium containing succinate as carbon source. In the absence 
of xylose, only 14 units of $\beta$-xylosidase activity were detectable, whereas in the presence of xylose a high $\beta$-xylosidase activity of 945 units was measured (Table 3, column 1), reflecting the repression of the xyn operon by the XylR protein. Next, we added other carbon sources in addition to succinate and repeated the measurements. Xylose as inducer for XylR was also included. In all cases, the XynB activities were reduced in comparison to the culture grown on succinate (Table 3, column 1). Succinate is known to cause no CCR in B. subtilis (Blencke et al., 2003; Galinier et al., 1999). Glucose caused the strongest reduction of XynB activity (135-fold), which is in agreement with previous data (Galinier et al., 1999). The sugars salicin, glycerol, mannitol and fructose also caused repression, but to a weaker extent (12- to 18-fold). Gluconate, sucrose and sorbitol repressed XynB activity eight-fold. Ribose, arabinose and maltose had only a two-fold effect. In conclusion, the substrates formed a hierarchy in their ability to exert CCR.

Table. 3. Catabolite repression of $\beta$-xylosidase by different carbon sources in various mutants. The values are the average of at least three independent experiments. Standard deviations are shown in parentheses.

\begin{tabular}{|c|c|c|c|c|c|c|c|}
\hline \multicolumn{8}{|c|}{ Enzyme activity in units/mg of protein } \\
\hline \multirow{4}{*}{$\begin{array}{l}\text { Carbon } \\
\text { source }^{\mathrm{a}}\end{array}$} & \multirow{4}{*}{$\begin{array}{c}168^{\mathrm{b}} \\
\text { wild-type }\end{array}$} & \multirow{4}{*}{$\begin{array}{l}\text { GP270 } \\
\Delta x y l R\end{array}$} & \multirow{4}{*}{$\begin{array}{c}\text { GP297 } \\
\Delta x y l R, \\
\Delta c r h\end{array}$} & \multirow{4}{*}{$\begin{array}{l}\text { GP284 } \\
\Delta x y l R, \\
p t s H 1\end{array}$} & \multirow{4}{*}{$\begin{array}{c}\text { GP287 } \\
\Delta x y l R, \\
\Delta c r h, \\
p t s H 1\end{array}$} & \multirow{4}{*}{$\begin{array}{l}\text { GP289 } \\
\Delta x y l R, \\
\Delta h p r K\end{array}$} & \multirow{4}{*}{$\begin{array}{l}\text { GP853 } \\
\Delta x y l R, \\
\Delta c c p A\end{array}$} \\
\hline & & & & & & & \\
\hline & & & & & & & \\
\hline & & & & & & & \\
\hline$-(\mathrm{CSE})$ & $945(281)$ & $1585(560)$ & $2748(540)$ & $2590(304)$ & $2397(214)$ & $2287(218)$ & $2142(384)$ \\
\hline Ribose & $497(138)$ & $939(164)$ & $1360(169)$ & $971(263)$ & $1346(151)$ & n. g. ${ }^{\mathrm{c}}$ & $1780(454)$ \\
\hline Arabinose & $414(136)$ & $600(153)$ & $876(35)$ & $713(100)$ & $1498(323)$ & $1257(70)$ & $1437(228)$ \\
\hline Maltose & 437 (127) & $489(32)$ & $488(105)$ & $710(81)$ & $2226(206)$ & $2023(408)$ & $2078(632)$ \\
\hline Gluconate & $116(11)$ & $201(31)$ & $378(64)$ & $244(46)$ & $1220(179)$ & $1163(170)$ & $1628(554)$ \\
\hline Sucrose & $126(20)$ & $205(12)$ & $203(13)$ & $309(88)$ & $2770(136)$ & $2271(292)$ & $2858(123)$ \\
\hline Salicin & $54(6)$ & $175(19)$ & $167(4)$ & $202(37)$ & $2850(180)$ & $2743(667)$ & 2049 (83) \\
\hline Sorbitol & $114(20)$ & $121(29)$ & $130(52)$ & $113(19)$ & $732(66)$ & $748(61)$ & 734 (51) \\
\hline Glycerol & $82(15)$ & $96(20)$ & 87 (6) & $135(17)$ & $2164(110)$ & $1273(160)$ & 1138 (127) \\
\hline Mannitol & $72(13)$ & $79(21)$ & $78(18)$ & 606 (190) & $2080(395)$ & $1689(269)$ & $1265(454)$ \\
\hline Fructose & $60(9)$ & $63(5)$ & $63(7)$ & $231(75)$ & 2045 (206) & $1638(32)$ & 1889 (147) \\
\hline Glucose & $7(3)$ & $44(10)$ & $60(21)$ & $173(56)$ & $2077(500)$ & $1679(421)$ & $1570(153)$ \\
\hline
\end{tabular}

${ }^{\mathrm{a}}$ added to CSE medium. ${ }^{\mathrm{b}}$ Xylose was added to induce $x y n P B$ expression. ${ }^{\mathrm{c}}=$ no growth 


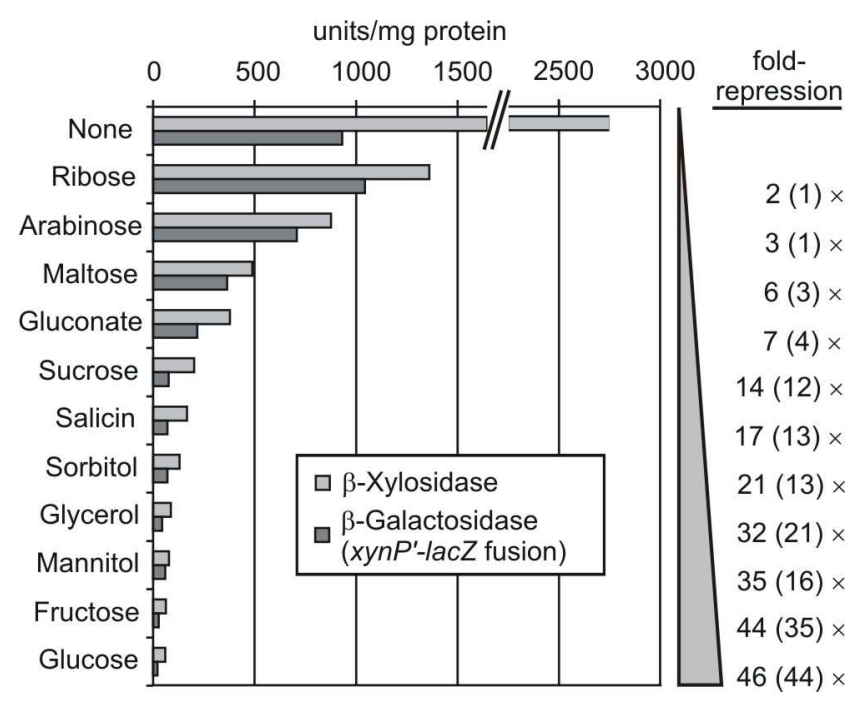

Figure. 10. Various carbohydrates repress the $x y n P B$ operon via the global CCR pathway. Shown are the $\beta$-xylosidase activities in the $\Delta x y l R \Delta c r h$ mutant GP297 (light grey bars), in which CCR exclusively relies on the activities of HPrK/P, CcpA and HPr. To demonstrate that the $\beta$ xylosidase activities reflect transcription from the $x y n P B$ promoter, the isogenic strain GP279 was used, which carries in addition a $x y n P^{\prime}-l a c Z$ fusion on the chromosome. The $\beta$-galactosidase activities produced in this strain are shown for comparison (dark grey bars). The repression factors relative to the activities determined in pure CSE medium (,none“) are depicted at the right.

To rule out that enzyme activity rather than synthesis of XynB was affected by the various carbon sources in these and the following experiments, we also performed similar experiments with isogenic strains carrying a transcriptional $x y n P^{\prime}-l a c Z$ fusion on the chromosome. The $\beta$-galactosidase activities in these strains correlated well with the corresponding $\beta$-xylosidase activities (Fig. 10 and data not shown). Somewhat lower repression factors were obtained in the LacZ assays in comparison to the $\mathrm{XynB}$ measurements, making the latter the more sensitive tool to measure CCR. In conclusion, the observed differences of $\mathrm{XynB}$ activities in this study reflect regulatory events taking place at the $x y n P B$ promoter.

\section{The XylR repressor protein contributes to CCR of $x y n P B$ expression exclusively in the presence of glucose}

As for many other systems, the $x y n P B$ operon underlies an operon-specific mechanism of $\mathrm{CCR}$ in addition to the general CCR pathway via CcpA. The XylR repressor also contributes to glucose repression of its target genes (Dahl et al., 1995; Kraus et al., 1994). Uptake of glucose generates glucose-6-phosphate, which binds to XylR and counteracts binding of the inducer xylose (Fig. 9). In principle, the repression of XynB activity by the other carbohydrates may also result from the combined activities of both mechanisms of CCR. To 
analyze this possible interference, we repeated our experiments with a $\Delta x y l R$ mutant. As expected, the $x y l R$ mutation caused complete de-repression of $x y n P B$ expression, i.e. high XynB activities were already detectable in the absence of the inducer xylose (Table 3, column 2). With respect to the available carbon sources, the enzyme activities followed a similar order as in the xylose-induced wild-type strain (Table 3, compare columns 1 and 2). In most cases, the activities were slightly higher in the $\Delta x y l R$ mutant, which can be explained by incomplete de-repression in the wild-type strain. In contrast, there was a 6-fold relief from repression when the cells grew on glucose, which reflects the extra-repression exerted by glucose-6-P. In conclusion, XylR only contributes to CCR of $x y n P B$ expression when glucose is utilized and is not relevant for CCR elicited by the other carbon sources.

\section{In the $\Delta x y l R$ mutant, CCR of $\beta$-xylosidase completely depends on the general pathway of CCR}

In the $\Delta x y l R$ mutant, the substrates still formed a hierarchical order in their ability to cause repression. We wanted to determine whether this order is caused exclusively by the global CCR mechanism or whether additional mechanisms are still involved. If only global CCR is involved, an interruption of the general CCR pathway is expected to completely relieve repression. To test this possibility, we used $\Delta x y l R$ strains, which additionally lacked HprK/P or CcpA or its cofactors Crh and HPr. In the latter case, a $\Delta c r h$ ptsHI mutant was used, which encodes an HPr mutant with a non-phosphorylatable alanine rather than a serine at position 46. The HPr-Ser46Ala protein is unable to participate in CcpA-mediated CCR but retains its function in PTS sugar uptake (Deutscher et al., 1994). All strains produced similar high $\beta$ xylosidase activities regardless of the carbon source, with the notable exception of sorbitol (Table 3, columns 5-7). On sorbitol a 3-fold repression was still detectable. On all other carbon sources, the elimination of the global regulators of CCR caused a complete relief from CCR. Hence, global CCR is responsible for the hierarchical order of repression caused by the different substrates in the $\Delta x y l R$ mutant.

\section{The amounts of HPrK, CcpA and HPr are not affected by the carbon source}

It is possible that the differences in repression exerted by the different substrates are caused by varying amounts of HPrK or CcpA or its co-factors in the cell. To address this possibility, we determined the amounts of these proteins in cells grown on the various carbon sources. Protein extracts were separated by SDS polyacrylamide gel electrophoresis and subjected to Western blot analyses using specific antisera. Only small differences in the amounts of the 
three proteins were detectable (Fig. 11). We also attempted to detect the Crh protein. However, although our antiserum readily detected purified Crh protein, no signals were obtained in cell extracts (data not shown). This observation is in agreement with our previous data suggesting that $\mathrm{Crh}$ is present in the cell in much lower amounts than HPr (Görke et al., 2004). In conclusion, the cellular amounts of the global CCR regulators HPrK/P, CcpA and HPr are not affected by the carbon source.

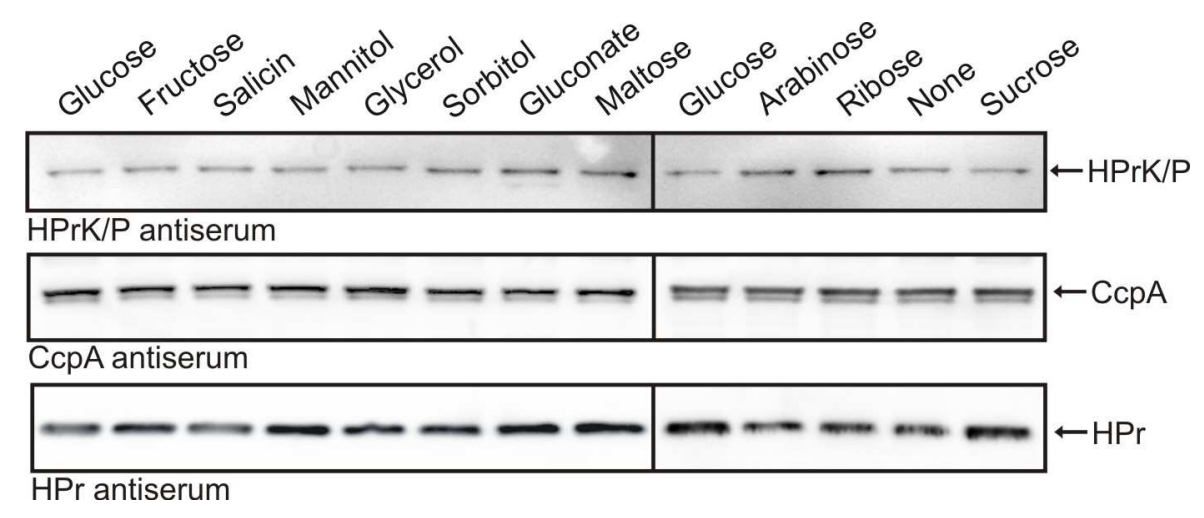

Figure. 11. The amounts of HPrK/P, CcpA and HPr are not affected by the utilized carbon source. Wild-type strain 168 was grown to exponential phase in CSE medium containing the indicated carbon sources and crude extracts were prepared. Of these protein extracts $10 \mu \mathrm{g}, 5 \mu \mathrm{g}$ and $2 \mu \mathrm{g}$ were loaded on $12.5 \%$ SDS-PAA gels for the detection of HPrK/P, CcpA and HPr, respectively. After gel electrophoresis proteins were blotted to a PVDF membrane and the proteins of interest were detected using specific antisera, respectively.

\section{HPr and Crh are not interchangeable in CcpA-mediated CCR}

In the $\Delta x y l R$ mutant, only global CCR is operative in $x y n P B$ repression. Hence, the degree of repression exerted by the individual substrates should be reflected by the total number of $\mathrm{HPr}(\mathrm{Ser}-\mathrm{P}) / \mathrm{CcpA}$ and $\mathrm{Crh}(\mathrm{Ser}-\mathrm{P}) / \mathrm{CcpA}$ complexes competent in DNA-binding. So far, it is unclear to which extent each of these complexes actually contributes to CCR via CcpA under a certain condition. Hence, it is possible that the cell selectively prefers or relies on one of these complexes depending on the carbon source. To address this question, we compared $\Delta x y l R \Delta c r h$ - and $\Delta x y l R p t s H 1$-mutants in which either one of the co-factors for CcpA is missing. The XynB activities in the $\Delta x y l R \Delta c r h$ mutant were very similar to those detected in the $\Delta x y l R$ mutant (Table 3, compare columns 2 and 3; Fig. 10). Therefore, Crh is dispensable for CCR exerted by the different carbon sources. Obviously, HPr can completely compensate for the loss of $\mathrm{Crh}$. A different result was obtained using the $\Delta x y l R$ ptsHl mutant. In this case, a 3- to 4-fold relief from repression was observed when the cells grew on glucose or fructose. On mannitol, the $\beta$-xylosidase activities were even 8-fold higher (Table 3, compare column 4 with columns 2 and 3 ). These sugars exert the strongest repression in the wild type and in the 
$\Delta x y l R$ mutant. In contrast, repression by all other substrates was not significantly affected by the ptsHl mutation. These data indicate that $\mathrm{Crh}$ is unable to substitute for HPr in the presence of substrates that exert a strong CCR. Hence, HPr is essential for CCR.

The extent to which a substrate causes repression correlates with the amount of $\operatorname{HPr}($ Ser-P) in the cell

The phosphorylation of HPr by the HPr kinase is a key step in the signaling pathway of CCR in B. subtilis. It is well established that significant amounts of $\mathrm{HPr}(\operatorname{Ser}-\mathrm{P})$ are present during growth on glucose, whereas little $\mathrm{HPr}(\mathrm{Ser}-\mathrm{P})$ is detectable in the absence of a sugar, i.e. in CSE minimal medium or LB (Ludwig et al., 2002; Monedero et al., 2001). However, the phosphorylation state of HPr has so far not been determined during growth in the presence of sugars other than glucose. In order to explore whether the strength of CCR exerted by a given substrate correlates with the amount of $\operatorname{HPr}(\operatorname{Ser}-\mathrm{P})$ in the cell, we determined the HPr phosphorylation state in vivo. To this end, we prepared extracts from cells grown on the various carbohydrates and subjected them to non-denaturing polyacrylamide gel electrophoresis, which allows the separation of the non-phosphorylated and phosphorylated forms of HPr. HPr was subsequently detected by Western blot analysis (Fig. 12).

As reported previously (Ludwig et al., 2002), HPr was present in three different forms when the cells grew in CSE medium: non-phosphorylated, single phosphorylated and doubly phosphorylated (Fig. 12, lane 1). HPr can be phosphorylated by the EI of the PTS on His-15 and by HPrK/P on Ser-46. To discriminate between both forms, aliquots of the extracts were incubated at $70^{\circ} \mathrm{C}$ before loading on the gel. Heating causes loss of phospho-histidine but not of phospho-serine bonds. As a result, the doubly phosphorylated HPr is converted to HPr(SerP) and $\mathrm{HPr}(\mathrm{His}-\mathrm{P})$ is converted to non-phosphorylated HPr (Fig. 12, even numbered lanes). From the quantification of the band intensities in both lanes and their comparison, it was possible to calculate the relative amount of each form of HPr (Table 4). In CSE medium, only $13 \%$ of total HPr were phosphorylated at Ser-46 and 5\% were doubly phosphorylated. The majority of HPr molecules were non-phosphorylated or phosphorylated at His-15. Very similar results were obtained when the cells grew on the weakly repressing sugars ribose, gluconate, arabinose or maltose. The level of $\operatorname{HPr}(\operatorname{Ser}-\mathrm{P})$ slightly increased up to $32 \%$ on gluconate, which is the most strongly repressing substrate among these carbohydrates (Fig. 12, top panel; Table 4). In all cases, HPr(His-P) and doubly phosphorylated HPr were the predominant forms. 
A very different result was obtained, when the cells grew on sugars that cause a stronger CCR, i.e. sorbitol, sucrose, salicin, mannitol, fructose and glucose. In these cases, almost no doubly phosphorylated HPr was detectable and heating of the extracts had almost no effect on the phosphorylation pattern (Fig. 12, lanes 11, 12 and 15-24). Hence, there was no detectable HPr(His-P) present in the cells. In contrast, $50-70 \%$ of all HPr molecules were phosphorylated at Ser-46 (Fig. 12, bottom panel; Table 4, rows 6-12). The only other detectable form was non-phosphorylated HPr. As an exception, glycerol behaved different from all other substrates: In contrast to the other strongly repressing substrates, it generated a somewhat lower level of HPr(Ser-P), i.e. 46\%. Moreover, no un-phosphorylated HPr could be detected. In contrast, doubly phosphorylated HPr was a predominant form in this case (Fig. 12, lanes $13,14)$.

In conclusion, sugars that exert strong (i.e. at least 10-fold, Fig. 10) repression of $\beta$ xylosidase activity also generate a high level of $\operatorname{HPr}(\operatorname{Ser}-\mathrm{P})$ in the cell, whereas in the presence of weakly repressing substrates the amount of $\mathrm{HPr}(\operatorname{Ser}-\mathrm{P})$ is significantly lower. In addition, the data suggest that the absence of histidine-phosphorylated HPr is a common feature of substrates that generate a strong CCR.

\section{His-15-dependent phosphorylation of HPr has no impact on CcpA-mediated CCR.} Interestingly, most of the carbohydrates that generated a strong CCR are substrates of the PTS. Glucose, fructose, mannitol, salicin and sucrose are all taken up by specific EIIs, which rely on $\mathrm{HPr}($ His-P)-dependent phosphorylation for this function. Hence, utilization of all these substrates drains away the phosphoryl-groups bound to His-15 of HPr. Accordingly, no HPr(His-P) can be detected when the cells grow on these substrates (Fig. 12 and Table 4). On the weakly repressing substrates ribose, arabinose and gluconate, which are non-PTS substrates, a considerable fraction of HPr is phosphorylated at its His-15 residue by Enzyme I (Table 4). Hence, it is conceivable that in these cases EI and HPrK compete for the phosphorylation of HPr and that CCR is weak, because EI-dependent phosphorylation limits the HPr(Ser-P) amount in the cell and thereby CCR.

To test this possibility, we used a $\Delta p t s I$ mutant in which HPr cannot get phosphorylated at His-15. If histidine phosphorylation of HPr limits CCR, one would expect an increased CCR by non-PTS substrates in this mutant. However, there was virtually no difference in CCR by non-PTS substrates between the $\Delta p t s I$ mutant and the wild-type strain (Fig. 13). In contrast, on PTS-sugars (and glycerol) an almost complete relief from CCR was observed in the $\Delta p t s I$ mutant. This was expected, because uptake and metabolism of these 
substrates are not possible in the $\Delta p t s I$ mutant. Hence, the cells actually use succinate for growth, which exerts no CCR. To support these data, we also investigated a mutant strain coding for an HPr-His15Ala variant. This strain exhibited $\beta$-xylosidase activities almost identical with those determined in the $\Delta p t s I$ mutant (our unpublished data). In conclusion, the phosphorylation state of $\mathrm{HPr}$ at its histidine residue has no direct impact on CcpA-mediated CCR. Therefore, solely different HPrK activities appear to account for the different CCR levels exerted by the various substrates.
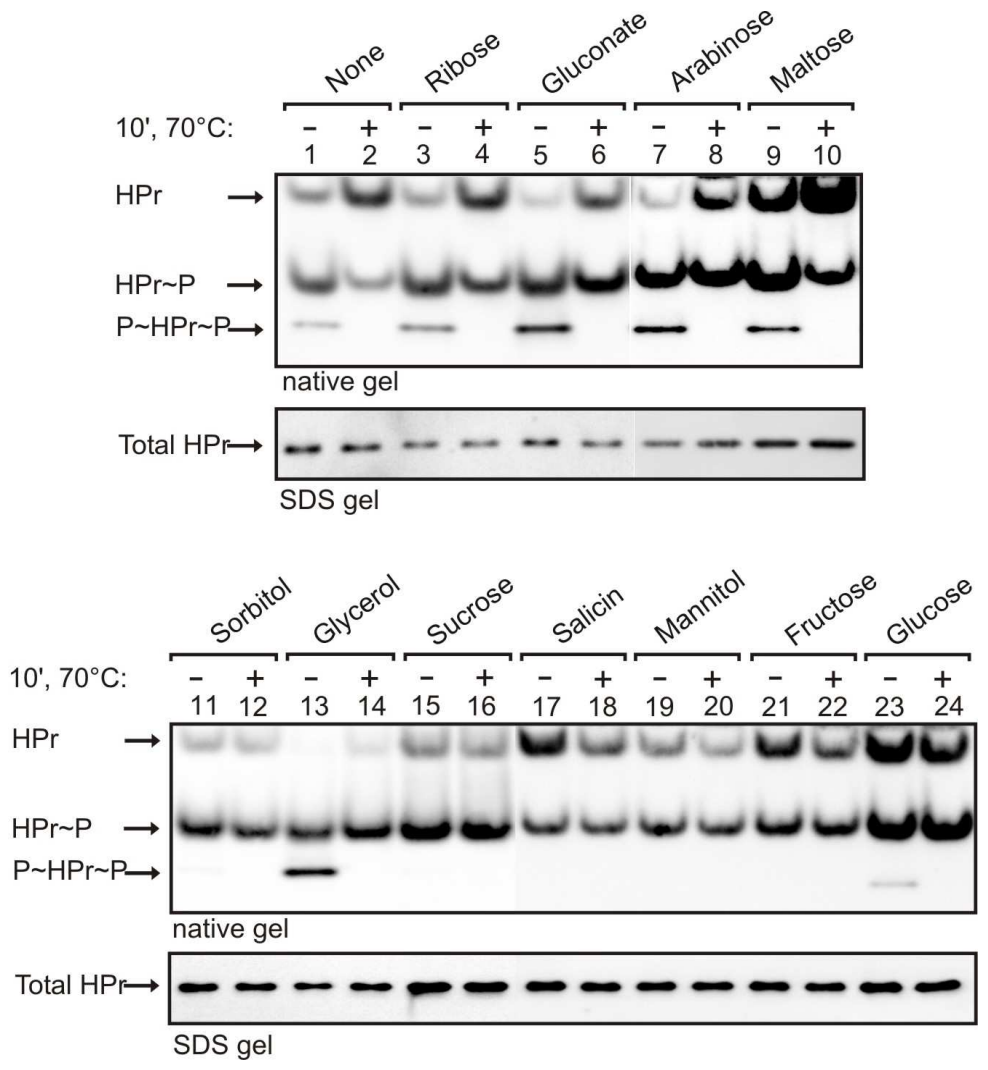

Figure. 12. Determination of the phosphorylation state of HPr in the presence of different carbon sources. Wild-type strain 168 was grown on CSE medium supplemented with the indicated carbohydrates. Protein extracts were prepared and separated on native 12\% PAA gels (top panels). HPr was subsequently detected by Western blotting (odd-numbered lanes). To discriminate between $\mathrm{HPr}(\mathrm{Ser}-\mathrm{P})$ and $\mathrm{HPr}(\mathrm{His}-\mathrm{P})$ an aliquot of each cell extract was heated $\left(70^{\circ} \mathrm{C}, 10 \mathrm{~min}\right)$ prior to loading (even-numbered lanes). This causes loss of the phospho-histidine bonds. The lysis buffer used affected the reliability of the Bradford assay for determination of protein concentrations. To account for the differences in the protein estimation and sample loading, $2 \mu \mathrm{g}$ of each protein extract (according to Bradford-assay) were separated in parallel by SDS-PAGE and total HPr was detected by Western blot analysis (bottom panels). 
Table. 4. Comparison of catabolite repression by different substrates, the relative amounts of the different HPr forms and the intracellular FBP concentration. Standard deviations are shown in parentheses.

\begin{tabular}{|c|c|c|c|c|c|c|c|}
\hline \multirow{2}{*}{$\begin{array}{l}\text { Carbon } \\
\text { source }^{\mathrm{a}}\end{array}$} & \multirow{2}{*}{$\begin{array}{l}\text { Fold- } \\
\text { repres- } \\
\text { sion }^{\mathrm{b}}\end{array}$} & \multicolumn{4}{|c|}{ Relative amount of $\mathrm{HPr}$ form $[\%]^{\mathrm{c}}$} & \multirow{2}{*}{$\begin{array}{c}\text { Fructose- } \\
\text { 1,6-bis-P } \\
{[\mathrm{mM}]}\end{array}$} & \multirow{2}{*}{$\begin{array}{c}\text { Growth rate } \\
\qquad \mu\left[\mathrm{h}^{-1}\right]\end{array}$} \\
\hline & & $\begin{array}{c}\text { HPr } \\
(\text { Ser-P) }\end{array}$ & $\begin{array}{c}\text { HPr } \\
\text { (His-P) }\end{array}$ & $\mathrm{HPr}$ & $\begin{array}{c}\text { HPr } \\
\text { (Ser- } \\
\text { P)(His- } \\
\text { P) }\end{array}$ & & \\
\hline - (CSE) & - & $13(3)$ & $40(14)$ & $42(19)$ & $5(2)$ & $1.8(0.5)$ & $0.29(0.03)$ \\
\hline Ribose & 2 & $27(1)$ & $37(6)$ & $25(9)$ & $11(4)$ & $6.5(0.1)$ & $0.76(0.07)$ \\
\hline Arabinose & 3 & $21(1)$ & $29(9)$ & $13(10)$ & $37(1)$ & $8.1(0.5)$ & $0.98(0.06)$ \\
\hline Maltose & 6 & $13(1)$ & $47(4)$ & $25(6)$ & $15(1)$ & $10.7(0.8)$ & $0.85(0.01)$ \\
\hline Gluconate & 7 & $32(10)$ & $29(4)$ & $9(2)$ & $30(9)$ & $12.3(3.6)$ & $0.94(0.09)$ \\
\hline Sucrose & 14 & 66 & 0 & 33 & 1 & $11.5(1.9)$ & $0.85(0.02)$ \\
\hline Salicin & 17 & $50(9)$ & 0 & $50(9)$ & 0 & $9.4(2.1)$ & $0.91(0.04)$ \\
\hline Sorbitol & 21 & $66(6)$ & 0 & $32(7)$ & $2(1)$ & $4.4(2.1)$ & $0.83(0.03)$ \\
\hline Glycerol & 32 & $46(3)$ & $12(6)$ & 0 & $42(4)$ & $4.3(1.6)$ & $0.94(0.11)$ \\
\hline Mannitol & 35 & $70(6)$ & 0 & $30(6)$ & 0 & $4.4(0.6)$ & $0.86(0.03)$ \\
\hline Fructose & 44 & $60(2)$ & 0 & $40(3)$ & 0 & $13.3(3.0)$ & $0.94(0.07)$ \\
\hline Glucose & 46 & $58(6)$ & 0 & $35(10)$ & $7(7)$ & $14.1(1.3)$ & $0.95(0.07)$ \\
\hline
\end{tabular}

added to CSE medium; for growth rate determinations CE-medium plus a single carbon source was used. ${ }^{\text {bin }}$ the $\Delta x y l R \Delta c r h$ mutant. ${ }^{c}$ mean values of at least two independent experiments are presented except for sucrose, which was performed once. 


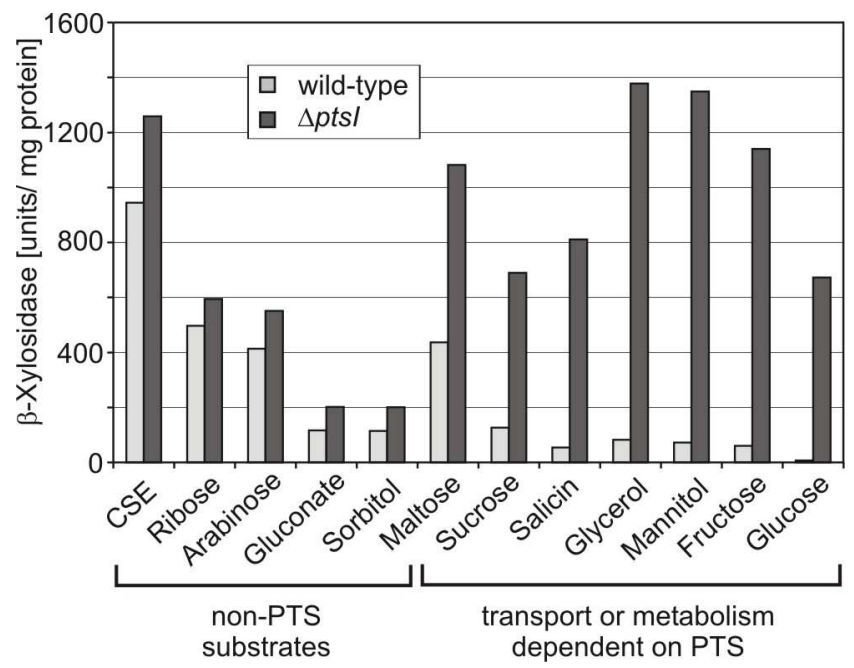

Figure. 13. Catabolite repression of $\beta$-xylosidase in a mutant lacking EI of the PTS. Strain GP864 $(\Delta p t s I)$ was grown in CSE medium supplemented with the indicated carbohydrates and the $\beta$ xylosidase activities were determined (dark grey columns). The corresponding activities in the wildtype strain 168 are shown for comparison (light grey columns).

\section{Activity of HPrK/P determines the level of CcpA-exerted CCR in Bacillus subtilis}

Finally, we wanted to confirm that a low HPr kinase activity is responsible for the weak CCR exerted by substrates like ribose, arabinose, gluconate or maltose. To this end, we used a strain, which expresses the mutant $h p r K-G 158 \mathrm{~A}$ allele rather than wild-type $h p r K$. The G158A exchange is located in the nucleotide binding Walker A motif of HPrK and abolishes phosphorylase activity in vitro (Hanson et al., 2002). As a result, HPrK-G158A behaves as a constitutive kinase, triggering the slow phosphorylation of HPr. In order to see whether this is indeed the case in vivo, we introduced a plasmid carrying hprK G158A into a mutant strain lacking the wild-type gene. A transformant carrying the empty expression plasmid served as a control. These transformants as well as the wild type strain were grown in CSE and CSE + glucose and the phosphorylation state of HPr was determined (Fig. 14). As expected, no $\mathrm{HPr}(\operatorname{Ser}-\mathrm{P})$ was detectable in the $\Delta h p r K$ mutant (Fig. 14, lanes 3, 4 and 9, 10). In the strain expressing the hprK G158A allele, the fraction of $\operatorname{HPr}(\operatorname{Ser}-\mathrm{P})$ increased to $38 \%$ when the cells grew in CSE, whereas only $13 \% \operatorname{HPr}(\operatorname{Ser}-\mathrm{P})$ were detectable in the wild-type (Fig. 14, compare lanes 1, 2 and 5,6). In the presence of glucose $43 \% \mathrm{HPr}(\operatorname{Ser}-\mathrm{P})$ were detectable in the mutant expressing the $h p r K$ G158A allele (Fig. 14, lanes 7, 8 and 11, 12). The wild-type strain produced 58\% $\mathrm{HPr}(\mathrm{Ser}-\mathrm{P})$ under these conditions (Fig. 14, lanes 7 and 8). In conclusion, the HprK G158A mutant triggers the phosphorylation of HPr at its Ser residue even in the absence of a repressing sugar. To see whether this increased fraction of HPr(SerP) also correlates with a stronger CCR, we determined the $\beta$-xylosidase activities in these 
strains (Table 5). In the $\Delta h p r K$ mutant carrying the empty expression plasmid, very high $\beta$ xylosidase activities were detectable, i.e. $x y n P B$ expression was relieved from CCR. In contrast, the strain expressing the $h p r K-\mathrm{G} 158 \mathrm{~A}$ allele produced only low activities in the range of 60 to 170 units. As an exception repression by glucose was somewhat stronger (21 units), which can be ascribed to the extra-repression by glucose-6-P via XylR. In conclusion, the data show that the HPrK-G158A variant phosphorylates HPr under all conditions and thereby triggers strong CCR on all substrates. This confirms that the different levels of CCR exerted by the various carbon sources (Fig. 10) solely result from different activities of $\mathrm{HPrK} / \mathrm{P}$.

Table. 5. Catabolite repression by the mutant HPrK/P-G158A protein lacking phosphorylase activity. The values are the average of at least two independent experiments.

\begin{tabular}{cccc}
\hline & Enzyme activity in units/mg of protein & \\
\hline Carbon source $^{\mathrm{a}}$ & $168^{\mathrm{b}}$ & GP858+pGP380 & GP858+pGP650 \\
& wild-type & $\Delta h p r K$ & $\Delta h p r K+h p r K-$ \\
& & & G158A \\
\hline- & $945(281)$ & $2077(323)$ & $178(10)$ \\
Ribose & $497(138)$ & n. g. ${ }^{\mathrm{c}}$ & $115(30)$ \\
Arabinose & $414(136)$ & $1175(50)$ & $138(27)$ \\
Maltose & $437(127)$ & $1265(82)$ & $142(17)$ \\
Sucrose & $126(20)$ & $1232(145)$ & $124(66)$ \\
Gluconate & $116(11)$ & $822(97)$ & $63(6)$ \\
Sorbitol & $114(20)$ & $990(52)$ & $90(22)$ \\
Glycerol & $82(15)$ & n. g. ${ }^{\mathrm{c}}$ & $70(15)$ \\
Mannitol & $72(13)$ & $1592(460)$ & $60(8)$ \\
Fructose & $60(9)$ & $1364(355)$ & $64(8)$ \\
Salicin & $54(6)$ & $981(168)$ & $46(27)$ \\
Glucose & $7(3)$ & $870(11)$ & $21(4)$
\end{tabular}

${ }^{\text {a added to CSE medium. }}{ }^{\mathrm{b}}$ xylose was added to induce $x y n P B$ expression. ${ }^{\mathrm{c}}=$ no growth 


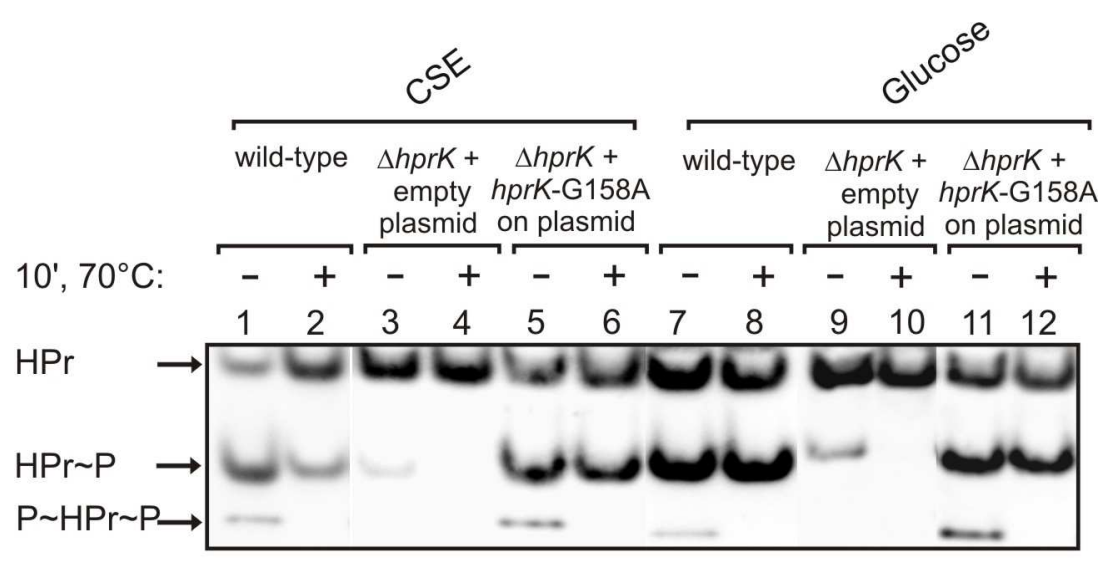

Figure. 14. The mutant HPrK/P-G158A protein phosphorylates HPr at Ser-46 even in the absence of a sugar. Strain GP858 $(\triangle \mathrm{hprK})$ carrying either plasmid pGP650 encoding the $h \mathrm{prK}$ G158A allele (lanes 5, 6, 11, 12) or the empty plasmid (lanes 3, 4, 9, 10) was grown in CSE or CSE + glucose. Crude extracts of these strains were subjected to non-denaturating PAGE and the phosphorylation state of HPr was determined as described for Fig. 12. For comparison, the wild-type strain 168 is also shown (lanes $1,2,7,8$ ).

\section{The intracellular FBP concentration and the strength of CCR exerted by a given carbohydrate do not strictly correlate}

FBP has been identified as a key metabolite modulating the activity of HPrK. To see, if there is a correlation between the intracellular FBP concentration and the level of CCR exerted by a given substrate, we determined the FBP concentrations (Table 4). In cells grown in pure CSE medium, only $1.8 \mathrm{mM}$ FBP was detectable, whereas the FBP concentration increased to 14.1 $\mathrm{mM}$ in the presence of glucose. These results are in perfect agreement with a previous study (Mijakovic et al., 2002). High FBP concentrations in the range of 9.4-13.3 mM FBP were also detectable in cells grown on the strongly repressing sugars fructose, salicin and sucrose. However, there was no strict correlation for the remaining substrates, e.g. the utilization of ribose, arabinose and maltose generated rather high FBP concentrations (6.5 - 10.7 mM FBP), whereas CCR exerted by these substrates was weak. In contrast, the FBP concentrations were lower (4.3 - $4.4 \mathrm{mM})$ on mannitol, glycerol and sorbitol, which all exert a strong CCR. In conclusion, the different levels of repression exerted by the different carbohydrates cannot be explained by the different intracellular FBP levels alone.

\section{Discussion}

In this work, we show that in addition to glucose many other carbohydrates cause carbon catabolite repression in B. subtilis. These substrates form a hierarchical order in their capacity to exert repression, suggesting that they trigger the formation of active CcpA complexes to 
different degrees (Table 3, Fig. 10). Our data show that the different carbon sources modulate the activities rather than the amounts of the proteins responsible for CCR. In fact, the data suggest a correlation between the ability of a sugar to cause repression and the phosphorylation state of HPr at its Ser-46 residue (Fig. 12). We could not observe any interference of the phosphorylation state of HPr at its His-15 residue with CcpA-mediated CCR of the $x y n P B$ operon, which served as a model system in this study. Our data suggest that at least in this case HPrK/P activity is the sole factor that accounts for the differences in repression by the various substrates. However, it should be emphasized that in other gene systems additional CcpA-independent mechanisms of CCR exist, which rely on HPr(His-P)dependent phosphorylation (for a review see: (Görke and Deutscher, 2007)).

The substrates form a hierarchy in their capacity to exert repression. The general CCR pathway determines this hierarchy, since the absence of either HprK/P or CcpA or its cofactors resulted in complete de-repression on all substrates. Intriguingly, there is a good correlation between the hierarchy in repression (this work) and the hierarchy of carbon source utilization reported by Monod based on diauxic growth experiments (Monod, 1942). Monod classified the carbon sources utilized by B. subtilis in two groups, A and B: When present in a mixture, the bacteria first utilize the substrates of group A, which include glucose, fructose, mannitol and sucrose. Subsequently the cells make use of the group B carbohydrates e.g. sorbitol, arabinose or maltose. Based on our observation that group A sugars exert strong CCR, whereas repression by group B sugars is weak, it appears reasonable that the diauxic growth behavior observed by Monod is caused by CcpA-mediated CCR. However, as an inconsistency we observed a strong repression of $x y n P B$ by sorbitol, which was assigned by Monod to group B. Interestingly, disruption of the CcpA-mediated CCR did not completely relieve $x y n P B$ from repression when the cells grew on sorbitol (Table 3). In contrast, other CcpA-controlled catabolic genes like rocG were completely de-repressed in $c c p A$ and $h p r K$ mutants grown on sorbitol (data not shown). Therefore, strong repression by sorbitol appears to be specific for the $x y n P B$ operon, which might explain the discrepancy to Monod's observations.

The Crh protein was completely dispensable for CCR exerted by the different substrates. A different result was obtained for a ptsH1 mutant, in which the Crh protein is the only potential effector for CcpA. In this case, there was a significant relief from CCR exerted by glucose, fructose and mannitol, but not from CCR exerted by the other sugars. In a previous study it was shown that in a $\mathrm{ptsHl}$ mutant synthesis of gluconate kinase and glucitol dehydrogenase is relieved from repression by glucose and mannitol but not by glycerol 
(Deutscher et al., 1994). Collectively, these observations suggest that Crh cannot substitute for HPr when the cells grow on substrates that cause a very strong CCR via CcpA. This might be reflected by the up to 100-fold lower synthesis rate of $\mathrm{Crh}$ and its 10 -fold lower affinity for CcpA, when compared with HPr (Görke et al., 2004). Alternatively, Crh might play a more specific role in CCR, when the cells use weaker repressing substrates. Recent work suggested that Crh might be more important for CCR during the transition to stationary phase (Inacio and de Sa-Nogueira, 2007). In conclusion, HPr rather than Crh is the relevant effector for CcpA in vivo, at least during the exponential growth phase.

According to the current model of the global CCR mechanism in B. subtilis, repression is brought about by the HPrK-catalyzed phosphorylation of HPr at its Ser-46 residue. Indeed, during growth on weakly repressing carbon sources only a minor fraction of HPr was phosphorylated at Ser-46, whereas the majority of HPr molecules were phosphorylated at this site on strongly repressing substrates. This clearly shows that the cell modulates the strength of CCR by dynamically triggering the HPrK/P-dependent (de)phosphorylation of HPr. Interestingly, large amounts of HPr(His-P) and also doubly phosphorylated HPr were formed, when the cells grew on weakly repressing substrates. The formation of significant amounts of doubly phosphorylated HPr in vivo is surprising. Previous in vitro studies suggested that $\mathrm{HPr}($ His-P) is a poor substrate for HPr kinase (Reizer et al., 1998). Moreover, a Ser46Asp exchange in HPr, which mimics phosphorylation at this site, was shown to block EIdependent phosphorylation in vitro (Reizer et al., 1989). These effects were explained by a diminished affinity for the second phosphoryl group delivering protein. Obviously, the in vitro data do not adequately reflect the situation in vivo.

Most of the strongly repressing substrates are transported by the PTS (glucose, fructose, mannitol, salicin and sucrose) and are therefore expected to dephosphorylate HPr at its His-15 residue, which is in agreement with our finding that no $\mathrm{HPr}(\mathrm{His}-\mathrm{P})$ was detectable in these cases. On the other hand, these observations raised the possibility that CCR is strong because dephosphorylation of HPr at His-15 makes the protein susceptible for HPr kinasecatalyzed phosphorylation, i.e. that EI and HPrK compete for phosphorylation of HPr. However, our experiments using mutants lacking EI were not in favor of this scenario (Fig. 13). Hence, the phosphorylation state of HPr at its His-15 residue is irrelevant for CcpAmediated CCR in B. subtilis. This is very different to E. coli, where the absence of EI leads to permanent repression of secondary catabolic genes (Postma et al., 1993). In E. coli, many substrates, which are taken up by the PTS cause CCR, because their transport not only dephosphorylates the general PTS proteins EI and HPr, but also the EIIA ${ }^{\text {Glc }}$ protein (Postma et 
al., 1993). In contrast, PTS transport activity has no direct effect on CCR in B. subtilis. Our data using the constitutive HPrK-G158A allele (Fig. 14 and Table 5) demonstrate that low HPr kinase activity limits CCR by weakly repressing carbon sources. Hence, different HPrK/P activities account for the different repression levels exerted by the various substrates. What are the molecular mechanisms that adjust the activity of HPrK/P to the available carbon source? The cells exhibited comparable growth rates on the different carbon sources except for succinate and ribose, on which growth was significantly slower (Table 4, last column). Therefore, it appears unlikely that the growth rate has a direct effect on HprK/P activity and therefore CCR. It is well known that the two antagonistic activities of HPrK/P are regulated by the concentrations of FBP, ATP and Pi. The central role of FBP for activity of the HPr kinase has been unequivocally proven in vivo and in vitro. In vivo, any mutation that prevents the formation of FBP results in a complete relief from CCR via CcpA, e.g. there is no CCR by glucose in mutants lacking the glycolytic enzymes glucose-6-phosphate isomerase or phosphofructokinase (Nihashi and Fujita, 1984). In vitro, HPr kinase activity is barely detectable below $1 \mathrm{mM}$ FBP. With higher FBP concentrations HPr kinase activity sharply increases and reaches a plateau at about 5 mM FBP (Jault et al., 2000; Reizer et al., 1998). In our experiments we detected a low FBP concentration of $1.8 \mathrm{mM}$ in the absence of a sugar (i.e. in CSE-medium), whereas in the presence of the various sugars, FBP concentrations in the range of $4.3-14.1 \mathrm{mM}$ were detected. Therefore, all sugars generated FBP levels, which are theoretically sufficient for a high HPr kinase activity. This suggests that the different levels of repression exerted by the different carbohydrates cannot be explained by the different intracellular FBP concentrations alone. Therefore, in addition to FBP other metabolites might account for the substrate-dependent differences of HPrK/P activity. Indeed, for Streptococcus bovis, an inverse correlation between $\mathrm{HPr}(\mathrm{Ser}-\mathrm{P})$ formation and the $\mathrm{Pi}$ concentration was observed in vivo (Asanuma and Hino, 2003). In addition, other metabolites like ATP, acetyl-phosphate and glyceraldehyde 3-phosphate were shown to modulate the activity of B. subtilis HPrK/P in vitro (Ramström et al., 2003).

Hierarchical regulation is a wide-spread phenomenon if bacteria have the choice between different substrates. If only few substrates can be used, sophisticated regulatory networks with multiple transcription factors allow the consecutive expression of the respective enzymes. This was observed for the choice of the electron acceptors for $E$. coli respiration (Unden and Bongaerts, 1997). However, individual regulators for the repression of genes that are lower in the hierarchy cannot be employed if the bacteria have to choose between a plethora of substrates. Accordingly, in E. coli and B. subtilis, the hierarchy of 
carbon sources in catabolite repression is established by a single signal, i.e. the control of the phosphorylation state of EIIA ${ }^{\mathrm{Glc}}$ and HPr, respectively ((Hogema et al., 1998), this work). 
4. Analysis of the hprK operon in Bacillus subtilis: Evidence for a role of a gene encoded downstream of $h p r K$ in carbon catabolite repression.

Author contributions:

This study was designed and interpreted by Kalpana Singh, Boris Görke and Jörg Stülke. All experiments were performed by Kalpana Singh. 


\section{Abstract}

HPr Kinase/phosphorylase (HPrK/P) is an ATP dependent Ser/ Thr kinase and controls the phosphorylation state of the phospho-carrier protein HPr at its Ser-46 residue in Bacillus subtilis and other Gram-positive bacteria. Under conditions of good carbon supply HPr(Ser-P) is formed and activates the global repressor protein CcpA by direct interaction. The $\mathrm{CcpA} / \mathrm{HPr}(\mathrm{Ser}-\mathrm{P})$ complex represses numerous catabolic genes, a regulatory mechanism known as catabolite repression. In addition to its role in CCR, HPr is also part of the phosphotransferase system, which catalyzes the uptake of a variety of sugars. HPrK/P is encoded by the first gene of a penta-cistronic operon. Here, we analysed the putative functions of the genes of the $\operatorname{hprK}$ operon. Initially, we observed that a polar $h p r K$ mutation can not be complemented by the ectopic expression of hprK. In this mutant, CCR was drastically relieved, but exclusively on PTS substrates. In agreement, there was also a lower amount of $\mathrm{HPr}(\mathrm{Ser}-\mathrm{P})$ present in the cell. On other carbohydrates, CCR was indistinguishable from the wild-type. Deletion analysis revealed that absence of lgt, the second gene of the operon, resulted in a slight relief from CCR regardless of the carbon source. Absence of the other genes downstream of $h p r K$ had no effect on HPrK/P activity. Since the lgt mutant and the mutant ectopically expressing $h p r K$ exhibited different CCR phenotypes, we conclude that over-expression of one of the genes downstream of $h p r K$ interferes with HPrK/P activity.

\section{Introduction}

Phosphorylation plays an important role in mediating signal transduction and regulation in bacteria (Hunter, 2000). A large number of physiological processes in bacteria are regulated by Ser/Thr kinases (Deutscher and Saier, Jr., 2005).The metabolite-controlled bifunctional HPr kinase/phosphorylase (HPrK/P) is one of the best studied Ser/Thr kinases in bacteria (Galinier et al., 1998; Poncet et al., 2004). HPrK/P triggers carbon catabolite repression (CCR) in Bacillus subtilis and other Firmicutes. CCR is a regulatory mechanism employed by bacteria in order to utilize the available nutrients in an economical way. In its natural habitat, B. subtilis often encounters a mixture of different carbon sources that can potentially be used. In this case, B. subtilis selectively utilizes the carbon source that permits the most rapid growth. For B. subtilis glucose is the preferred carbon source (Monod, 1942). During utilization of glucose, the genes required for the utilization of secondary substrates are not expressed. This phenomenon is referred to as CCR (Görke and Stülke, 2008). 
HPrK/P catalyses both, the phosphorylation and dephosphorylation of the histidine-containing protein (HPr) and of its homologue Crh (catabolite repression $\underline{\text { HPr) }}$ ) at a serine residue (Ser46) (Galinier et al., 1998; Kravanja et al., 1999). This phosphorylation triggers the interaction of HPr and Crh with the global transcriptional regulator protein $\mathrm{CcpA}$ (catabolite control protein A) (Schumacher et al., 2004; Schumacher et al., 2006; Seidel et al., 2005). The resulting $\operatorname{HPr}(\operatorname{Ser}-\mathrm{P}) / \mathrm{CcpA}$ and $\mathrm{Crh}(\operatorname{Ser}-\mathrm{P}) / \mathrm{CcpA}$ complexes bind to operator sites on the DNA called cre (ㅁatabolite repression elements) (Weickert and Chambliss, 1990). In most cases, the cre sites are located in promoter regions. Binding of the CcpA complex usually abolishes promoter activity and thereby represses gene expression. In vivo, HPr is the relevant co-factor for CcpA, whereas Crh appears to be dispensable (Singh et al., 2008).

In vitro experiments demonstrated that the activities of $\mathrm{HPrK} / \mathrm{P}$ are allosterically controlled by metabolites. The kinase activity of $B$. subtilis HPrK/P is stimulated by fructose 1,6-bisphosphate (FBP), whereas the phosphorylase activity prevails in the presence of high inorganic phosphate (Pi) concentrations (Jault et al., 2000). From in vitro experiments using Enterococcus faecalis $\mathrm{HPrK} / \mathrm{P}$, it was concluded that under in vivo conditions kinase activity might dominate when the concentrations of ATP and FBP are high in the cell, whereas the dephosphorylation should be prevalent when the concentration of Pi increases (Kravanja et al., 1999)

HPr, the substrate of $\mathrm{HPrK} / \mathrm{P}$, is also a part of the phosphoenolpyruvate (PEP): carbohydrate phosphotransferase system (PTS). The PTS is an important carbohydrate uptake system in many bacteria. In this system, the general phosphotransferases EI and HPr transfer phosphoryl groups derived from PEP to the various sugar-specific Enzyme II (EII) transporters, which phosphorylate their substrates during the uptake step. In this case, HPr is phosphorylated at histidine-15 (His-15). The HPr homolog Crh lacks His-15 and is therefore unable to participate in sugar transport (Galinier et al., 1997). A recent analysis demonstrated that PTS-sugar transport, i.e. (de)phosphorylation of HPr at His-15 does not interfer in vivo with the CcpA-mediated pathway of CCR, i.e with phosphorylation of HPr at Ser-46 (Singh et al., 2008).

In B. subtilis, gene hprK encoding the HPr kinase/phosphorylase, is the first gene of a penta-cistronic operon, which is conserved in many Firmicutes (Boël et al., 2003). Downstream of $h p r K$, the genes lgt, yvoD, yvoE and yvoF are present (Fig. 15) (Reizer et al., 1998; Deutscher, 2006). The second gene of the operon, lgt, codes for the prolipoprotein diacylglyceryl transferase, which catalyzes the first reaction of lipomodification of bacterial lipoproteins (Leskelä et al., 1999). The lipid on lipoproteins is covalently bound to a cysteyl 
residue and anchors the protein molecules to the outer surface of the cell membrane. The genes $y v o D$ and $y v o E$ code for an uncharacterized hydrophobic protein and a pyrophosphate, respectively. Sequence comparison indicates that $y v o E$ encodes a homologue of the phosphoglycolate phosphatase of E. coli. Phosphoglycolate phosphatase in E. coli is involved in the dissimilation of the intracellular 2-phosphoglycolate formed in the DNA repair of 3'phosphoglycolate ends (Teresa et al., 2003). During the initial characterization of the protein encoded by $y v o E$, it was mistakenly assumed to be the $\mathrm{HPr}(\operatorname{Ser}-\mathrm{P})$ phosphatase (Galinier et al., 1998). Later it was established that dephosphorylation of $\operatorname{HPr}(\operatorname{Ser}-\mathrm{P})$ is also catalyzed by HPrK/P, demonstrating that it is a bifunctional enzyme (Kravanja et al., 1999). However, recent work suggested that $\mathrm{YvoE}$ might play yet another role in regulating the $\mathrm{HPr}(\mathrm{Ser}-\mathrm{P})$ level in the cell. It was demonstrated in vitro that the dephosphorylation of $\operatorname{HPr}(\operatorname{Ser}-\mathrm{P})$ by $\mathrm{HPrK} / \mathrm{P}$ requires $\mathrm{Pi}$ as a substrate and generates pyrophosphate (PPi) as product (Mijakovic et al., 2002). PPi can also act as phosphate donor for the phosphorylation of HPr by HPrK/P, at least in vitro. Therefore, it was suggested that in vivo the intracellular PPi level could affect the phosphorylation of $\mathrm{HPr}$ at Ser-46. However, it was demonstrated that YvoE has pyrophosphatase activity suggesting that this protein removes the pyrophosphate generated by the phosphorylase activity of HPrK/P (Mijakovic et al., 2002). YvoE is not present in all Firmicutes, e.g. Staphylococcus aureus and S. epidermis lack the corresponding gene. However, these bacteria encode several YvoE homologues, which potentally could substitute for YvoE. The last gene of the hprK operon in B. subtilis is yvoF. The C-terminal half of the corresponding protein shares homology with acetyl transferases, including chloramphenicol acetyltransferase, serine acetyltranferase and thiogalactoside acetyltransferase (Reizer et al., 1998). The function of this gene is unknown.

In this work, we analysed the putative roles of the genes encoded downstream of hprK for the activity and function of HPrK/P. Initially, we observed that a polar hprK mutation cannot be complemented by the expression of $h p r K$ from an ectopic locus. In this case, CCR triggered by a subset of carbohydrates, i.e. salicin, mannitol, fructose and glucose could not be restored. In contrast, other carbohydrates, e.g. gluconate, glycerol or sorbitol exerted CCR in this strain indistinguishable from the wild-type. A detailed deletion analysis revealed that mutation of lgt affects CCR, i.e. absence of this gene resulted in a slight relief from CCR regardless of the carbon source. Absence of the other genes downstream of $h p r K$ had no effect on HPrK/P activity. Since the lgt mutant and the mutant expressing $h p r K$ ectopically exhibited different CCR phenotypes, we conclude that over-expression of one of the genes downstream of $h p r K$ interferes with HPrK/P activity. 


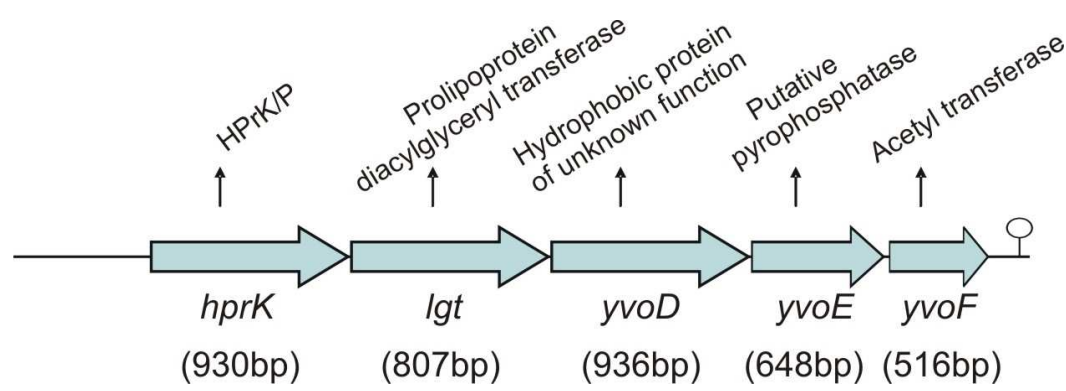

Figure. 15. Schematic representation of the $\mathbf{h p r K}$ operon of $\boldsymbol{B}$. subtilis. The protein product of each gene of the $h p r K$ operon is indicated by an arrow. The putative terminator is depicted by a lollipop (Reizer et al., 1998). Numbers in parentheses indicate the length of each open reading frame in base pairs (ORF). The operon is drawn to scale.

\section{Materials and Methods}

\section{Bacterial strains and growth conditions}

The B. subtilis strains used in this study are listed in Table. 6. E. coli DH5 $\alpha$ was used for plasmid propagation (Sambrook and Russel, 2001). E. coli was grown in Luria-Bertani medium and transformants were selected on plates containing ampicillin $\left(100 \mu \mathrm{g} \mathrm{ml}^{-1}\right) . B$. subtilis was grown in CSE medium which is C minimal medium supplemented with $6 \mathrm{~g} / \mathrm{l}$ potassium succinate and $8 \mathrm{~g} / \mathrm{l}$ potassium glutamate (Martin-Verstraete et al., 1995) and auxotrophic requirements (at $50 \mathrm{mg} \mathrm{l}^{-1}$ ). Carbon sources were used at a concentration of $0.5 \%$ (w/v). LB and SP plates were prepared by addition of $17 \mathrm{~g}$ Bacto agar/l (Difco).

\section{DNA manipulation}

Transformation of E. coli and plasmid extraction was performed using standard procedures (Sambrook and Russel, 2001). Restriction enzymes and DNA polymerases were used as recommended by the manufacturers. Plasmid pGP634 was constructed by inserting a DNA fragment encompassing $h p r K$ and $700 \mathrm{bp}$ of its upstream region,into the unique EcoRI- and SacI- sites of plasmid pAC6 (Stülke et al., 1997). The insert was amplified using primer SK3 and SK4. See table 7 for the list of oligonucleotides used in this study.

\section{Construction of strains and characterization of phenotypes}

Strains carrying deletion of the various genes of the $h p r K$ operon were generated using the Long Flanking homology PCR protocol as described previously (Wach, 1996). Briefly, cassettes carrying the kanamycin resistance gene were amplified from plasmid pDG780 using primers kan cassette fwd and kan cassette rev (Guerout-Fleury et al., 1995). Next DNA 
fragments of $\sim 1000 \mathrm{bp}$ were amplified carrying the sequences upstream and downstream of the region to be deleted, respectively. See Table. 7 for the oligonucleotides used for this purpose. The joining of the two fragments to the resistance cassette was performed in a second PCR as described previously (Jordan et al., 2006). The resulting PCR products were used to transform B. subtilis strains GP270 ( $\Delta x y l R)$. The integrity of the regions flanking the integrated resistance cassettes was verified by sequencing PCR products of $\sim 1000 \mathrm{bp}$, which were obtained by PCR using chromosomal DNA of the respective mutants. The resulting strains were GP851 ( $\Delta x y l R,[\Delta l g t, \Delta y v o D, \Delta y v o E, \Delta y v o F])$, GP852 ( $\Delta x y l R, \Delta y v o E)$, GP859

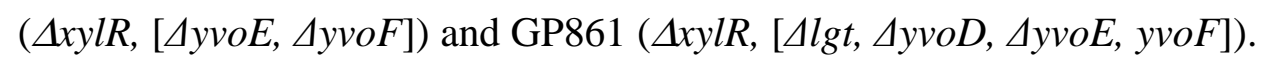

Strain GP290 carrying the $h p r K$ gene ectopically integrated in the amyE locus was constructed by transformation of strain GP270 ( $\Delta x y l R)$ with plasmid pGP634, which was linearized by SacI digestion. Plasmid pGP634 allows integration of the $h p r K$ gene into the $a m y E$ locus by a double crossing over. On this plasmid the cloned insert is flanked by sequences corresponding to the $a m y E-5$ ' and $a m y E-3$ ' regions. The $a m y E$ locus codes for nonessential $\alpha$-amylase and can be used for integration of foreign DNA. A successful recombination disrupts the $a m y E$ gene, which was confirmed by an iodine/starch test for amylase activity. For this purpose strain GP290 was grown on SP medium supplemented with hydrolyzed starch (10 g/ 1). Starch degradation was detected by sublimating iodine onto the plates.

Table. 6. $B$. subtilis strains used in this study

\begin{tabular}{|c|c|c|c|}
\hline Strain & Genotype & Source $^{\mathrm{a}}$ & \\
\hline 168 & $\operatorname{trpC2}$ & Laboratory stock & \\
\hline GP270 & trpC2 xylR::ermC & Singh et al., 2008 & \\
\hline GP289 & $\operatorname{trpC} 2$ xylR::ermC $\Delta$ hprK::aphA3 & Singh et al., 2008 & \\
\hline GP290 & $\operatorname{trpC} 2$ xylR::ermC $\triangle$ hprK::aphA3 amyE:: hprK cat & pGP634 $\rightarrow$ GP289 & \\
\hline GP851 & $\operatorname{trpC} 2$ xylR::ermC $\Delta$ lgt-yvoF::aphA3 & $\begin{array}{l}\text { LFH PCR product } \\
\text { GP270 }\end{array}$ & $\rightarrow$ \\
\hline GP852 & trpC2 xylR::ermC $\triangle$ yvoE::aphA3 & $\begin{array}{l}\text { LFH PCR product } \\
\text { GP270 }\end{array}$ & $\rightarrow$ \\
\hline GP859 & $\operatorname{trpC} 2$ xylR::ermC $\triangle y v o E-y v o F:: a p h A 3$ & $\begin{array}{l}\text { LFH PCR product } \\
\text { GP270 }\end{array}$ & $\rightarrow$ \\
\hline GP861 & $\operatorname{trpC} 2$ xylR::ermC $\triangle y v o D-y v o F:: a p h A 3$ & $\begin{array}{l}\text { LFH PCR product } \\
\text { GP270 }\end{array}$ & $\rightarrow$ \\
\hline
\end{tabular}

${ }^{\mathrm{a}}$ Arrows indicate construction by transformation 
Table. 7. List of oligonucleotides used in this study

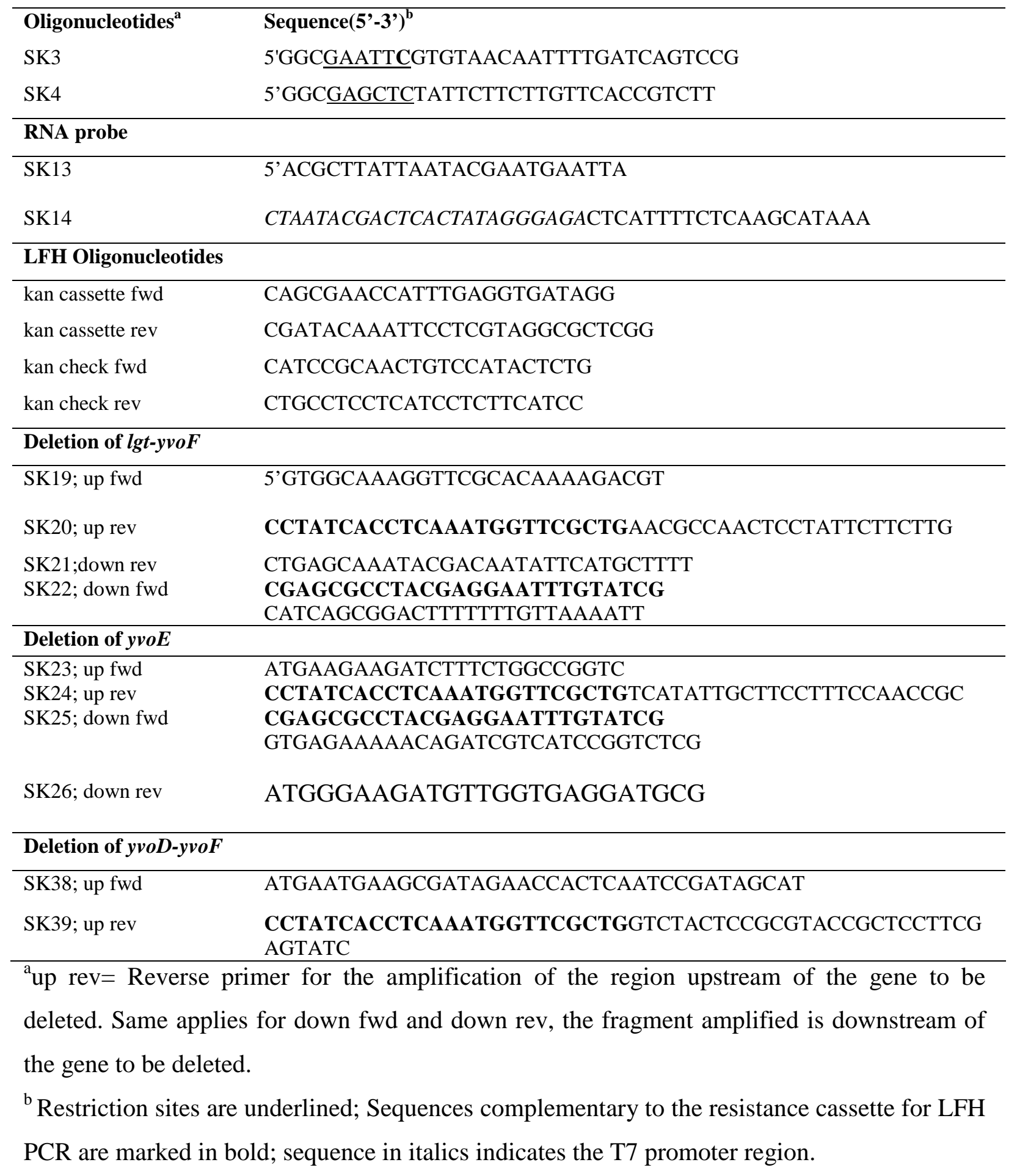

\section{Transformation and enzyme assays}

B. subtilis was transformed using the two step protocol (Kunst and Rapoport, 1995). Transformants were selected on SP plates containing kanamycin $\left(5 \mu \mathrm{g} \mathrm{ml}^{-1}\right)$ or erythromycin plus lincomycin $\left(2 \mu \mathrm{g} \mathrm{ml}^{-1}\right.$ and $25 \mu \mathrm{g} \mathrm{ml}^{-1}$ respectively). Cells were harvested in exponential 
growth phase at an $\mathrm{OD}_{600}$ of 0.6-0.8. $\beta$-xylosidase activities were measured in cell extracts using p-nitrophenyl xyloside as substrate (Lindner et al., 1994).

\section{Western blot analysis}

For western blot analysis crude cell extracts were prepared and were separated by either SDS or native PAGE, depending on the experimental requirements and transferred to a polyvinylidene difluoride membrane (PVDF, BioRad). The proteins were detected with rabbit polyclonal antisera against $\mathrm{HPr}$, RocG, HPrK of B. subtilis or B. megaterium respectively (Commichau et al., 2008; Hanson et al., 2002; Monedero et al., 2001). The purified streptagged RocG was kindly provided by Fabian Commichau. The antibodies were visualized by using anti-rabbit IgG-AP secondary antibodies (Promega, Madison, and U.S.A) and the CDP star detection system (Roche Diagnostics).

\section{Phosphorylation state of HPr in vivo}

HPr phosphorylation was assayed by Western blot analysis as follows. Cells were grown in CSE minimal medium supplemented with $0.5 \%$ glucose to an O.D of 0.6 followed by an addition of $12 \mathrm{M} \mathrm{HCl}$ to adjust the $\mathrm{pH}$ of culture to 4.5. Cells were disrupted using a French press, and crude extracts were prepared as described before (Ludwig et al., 2002). Proteins were loaded on a $10 \%$ native polyacrylamide (PAA) gel, allowing the separation of phosphorylated forms of protein from the non-phosphorylated ones. To distinguish the $\mathrm{HPr}($ His-P) from $\mathrm{HPr}($ Ser-P), we took advantage of the heat instability of the phospho-amide bond. An aliquot of each crude extract was incubated at $70^{\circ} \mathrm{C}$ for $10 \mathrm{~min}$ before separation on PAA gel. After electrophoresis, the proteins were blotted to a PVDF membrane. The different forms of HPr were detected using antibodies directed against B. subtilis HPr (Monedero et al., 2001).

\section{Northern analysis}

RNA was prepared by the modified 'mechanical disruption protocol' described previously (Ludwig et al., 2001). Briefly, $20 \mathrm{ml}$ of cells were harvested at the exponential phase. After mechanical cell disruption, the frozen powder was immediately re-suspended in $3 \mathrm{ml}$ lysis buffer [4 M guanidine isothiocyanate, $0.025 \mathrm{M}$ sodium acetate, $\mathrm{pH} 5.3,0.5 \% \mathrm{~N}$ laurylsarcosine (wt/vol)]. Subsequently, total RNA was extracted using the RNeasy Mini kit according to the manufacturers protocol (Qiagen, Germany). Digoxigenin-labelled RNA probes directed against the $y v o E$ transcript were obtained by in vitro transcription using $\mathrm{T} 7$ 
RNA polymerase (Roche Diagnostics) and a DNA-fragment as template, which was obtained by PCR using primers SK13 and SK14. The reverse primer SK14 contained a T7 RNA polymerase recognition sequence. In vitro RNA labelling, hybridization and signal detection were carried out according to the instructions of the manufacturer (DIG RNA labelling kit and detection chemicals; Roche Diagnostics). Total RNA was separated on a $0.8 \%$ agarose gel followed by transfer to a nylon membrane. The desired signals were detected after hybridisation with the RNA probe directed against yvoE.

\section{Results}

\section{Construction of a strain, which expresses $h p r K$ ectopically from the amyE locus}

We wanted to determine whether a deletion of the $h p r K$ gene can be complemented by the ectopic expression of $h p r K$ from a locus in trans. For this purpose, we constructed a mutant in which $h p r K$ was expressed from an ectopic location under the control of its native promoter while the native copy of the gene was absent. To achieve this, the $h p r K$ gene including 700 base pairs upstream was inserted into the amyE locus on the chromosome. At the same time, 209 codons of the endogenous $h p r K$ gene were deleted and replaced by a kanamycin cassette. To confirm, that $h p r K$ was indeed expressed from its ectopic location in this mutant (strain GP290), we performed a Western blot analysis using antiserum directed against HPrK/P. A set of three independent experiments suggested that similar amounts of HPrK/P is present in GP290, which expresses the hprK from the ectopic locus and the corresponding wild type
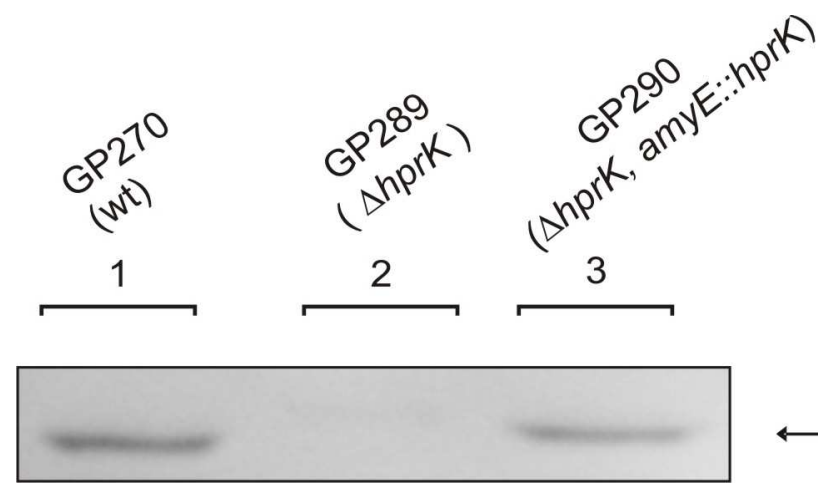

$\longleftarrow \alpha \operatorname{HPrK}$

Figure. 16. Western blot to confirm the expression of the ectopic hprK gene in strain GP290. The strains carrying the mutations as indicated at top were grown in CSE minimal medium supplemented with $0.5 \%$ glucose and were harvested in the logarithmic phase $\left(\mathrm{OD}_{600} 0.6-0.8\right)$. In each case, $15 \mu \mathrm{g}$ of total cell protein were separated on a 10\% SDS-PAA-gel and blotted onto PVDF membrane. HPrK/P was detected using antibodies raised against HPrK/P. No signal was detected in strain GP289 (lane2), confirming the absence of HPrK/P. 
strain GP270 (Fig. 16., compare lanes 1 and 3). No HPrK/P was detectable in the uncomplemented $\Delta h p r K$ mutant GP289 (Fig. 16, lane 2). In conclusion, the ectopic $h p r K$ gene is properly expressed.

\section{The ectopic expression of $h p r K$ does not restore CCR in the hprK::aphA3 mutant}

In strains lacking a functional HPrK/P, gene expression is completely relieved from CCR, i.e. the repressive effect of preferred carbon sources on the expression of secondary catabolic genes is abolished (Galinier et al., 1998; Hanson et al., 2002; Martin-Verstraete et al., 1999; Reizer et al., 1998; Singh et al., 2008). To check if the ectopically expressed HPrK/P is functionally active, we determined the carbon catabolite repression $\beta$-xylosidase (XynB) activity by different carbon sources. We have previously shown that activity of $\mathrm{XynB}$ is a perfect reporter for the CcpA-mediated pathway of CCR (Singh et al., 2008). However, this requires deletion of the $x y l R$ repressor gene, which encodes the dedicated substrate dependent regulator of $x y n B$ expression. In $x y l R$ mutants, the expression of $x y n B$ exclusively relies on the activity of CcpA. Therefore, the $x y l R$ gene was deleted in all strains used throughout this study. The $\Delta x y l R$ single mutant served as the "wild-type" control in these experiments.

As can be seen from the data presented in Table. 8, the wild-type strain produced high XynB activities in CSE-medium, i.e. when succinate, which exerts no CCR, was the single carbon source. The presence of an additional carbohydrate reduced the XynB activity (Table 8, column 1). Glucose, fructose and mannitol had the strongest repressing effect, whereas repression by other carbon sources was weaker, as reported recently (Singh et al., 2008). In the $\Delta h p r K$ mutant high $\mathrm{XynB}$ activities were detected in all cases, reflecting the release from CCR (Table 8, column 2; Singh et al., 2008).

In the $[\Delta h p r K$ amyE::hprK] mutant, which expresses hprK ectopically, CCR was perfectly restored when the cells grew on arabinose, maltose, gluconate, glycerol or sorbitol (Table 8, compare columns 1 and 3). Surprisingly, CCR was not restored to wild-type levels in the presence of salicin, mannitol, fructose or glucose. In these cases, a 5- to 14-fold relief from CCR was still detectable. In conclusion CCR of XynB activity was not restored on those substrates, which exert the strongest repression in the wild-type strain.

It could be possible that reduced CCR observed in the [ $\Delta$ hprK amyE::hprK] mutant is confined to the xyn operon only. To address this possibility, we investigated CCR of rocG expression. The rocG gene codes for the enzyme glutamate dehydrogenase, RocG (Belitsky and Sonenshein, 1998). The expression of $\operatorname{roc} G$ is induced by arginine and strongly repressed. 
Table. 8. Catabolite repression of $\beta$-xylosidase by different carbon sources in various mutants. Standard deviations are shown in parentheses except for the measurements done once.

$\beta$-xylosidase activity in units/mg of protein

\begin{tabular}{|c|c|c|c|}
\hline Carbon & GP270 & GP289 & GP290 \\
\hline source $^{a}$ & wild-type & $\Delta h p r K$ & $\triangle h p r K$, amyE: $h p r K$ \\
\hline- & $1585(560)$ & $2287(218)$ & $2317(112)$ \\
\hline Arabinose & $600(153)$ & $1257(70)$ & $680(96)$ \\
\hline Maltose & $489(32)$ & 2023(408) & $665(77)$ \\
\hline Gluconate & $201(31)$ & 1163(170) & $286(35)$ \\
\hline Glycerol & $96(14)$ & $1273(160)$ & $98(4)$ \\
\hline Sorbitol & $121(29)$ & $748(61)$ & $186(2)$ \\
\hline Salicin & $175(14)$ & 2743(667) & 838 \\
\hline Mannitol & $83(17)$ & $1689(269)$ & 958 \\
\hline Fructose & $66(6)$ & $1638(32)$ & $340(44)$ \\
\hline Glucose & $44(10)$ & $1679(421)$ & $610(270)$ \\
\hline
\end{tabular}

a added to CSE medium $(0.5 \%)$.

in the presence of preferred carbon sources such as glucose. This glucose-mediated CCR depends on CcpA (Belitsky et al., 2004). To study CCR of the rocG gene, the various mutant strains were grown in arginine-containing CSE-medium in the absence or presence of glucose. The cells were harvested and after separation of the protein extracts by SDS-PAGE, a Western blot analysis using antibodies directed against RocG was performed. As expected from previous data (Commichau et al., 2007), RocG was readily detectable in the wild-type strain, but its synthesis was strongly repressed upon addition of glucose (Fig. 17, compare lanes 1 and 2). The repression by glucose was abolished in the $\Delta h p r K$ mutant (Fig 17, compare lanes 3 and 4). The same result was obtained for the [ $\triangle h p r K$ amyE: $h p r K]$ mutant, which expresses $h p r K$ from the amyE locus. This demonstrates that the weak CCR by glucose 
observed in this mutant is not restricted to $x y n B$, but does also apply to other genes such as $\operatorname{roc} G$.

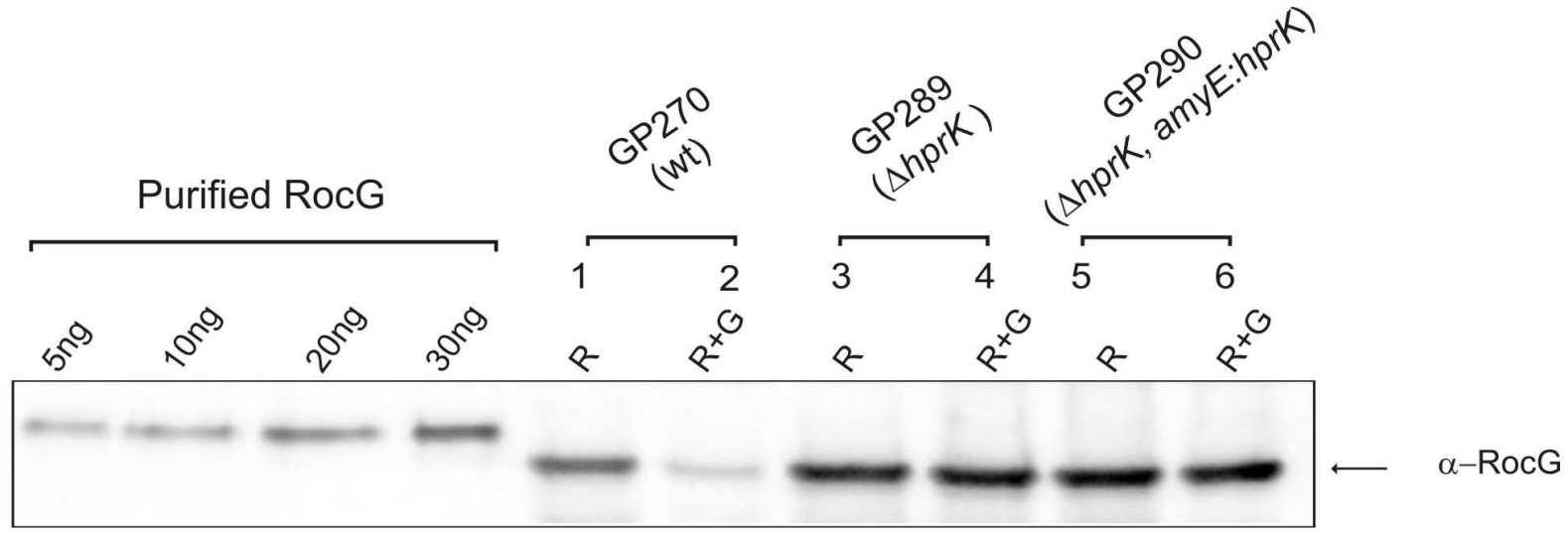

Figure. 17. Western blot analysis to determine CCR of RocG synthesis in various mutants. $15 \mu \mathrm{g}$ total cell protein were separated on a 10\% SDS-PAA-gel and blotted onto a PVDF membrane. The specific signal was detected using antibodies raised against purified B. subtilis RocG (Commichau et $a l ., 2008)$. Strains were grown in CSE minimal medium supplemented with $0.5 \%$ arginine (R) in the absence or presence of $0.5 \%$ w/v glucose $(\mathrm{G})$. Purified Strep tagged RocG loaded in various amounts (5- $30 \mathrm{ng}$ ) served as control.

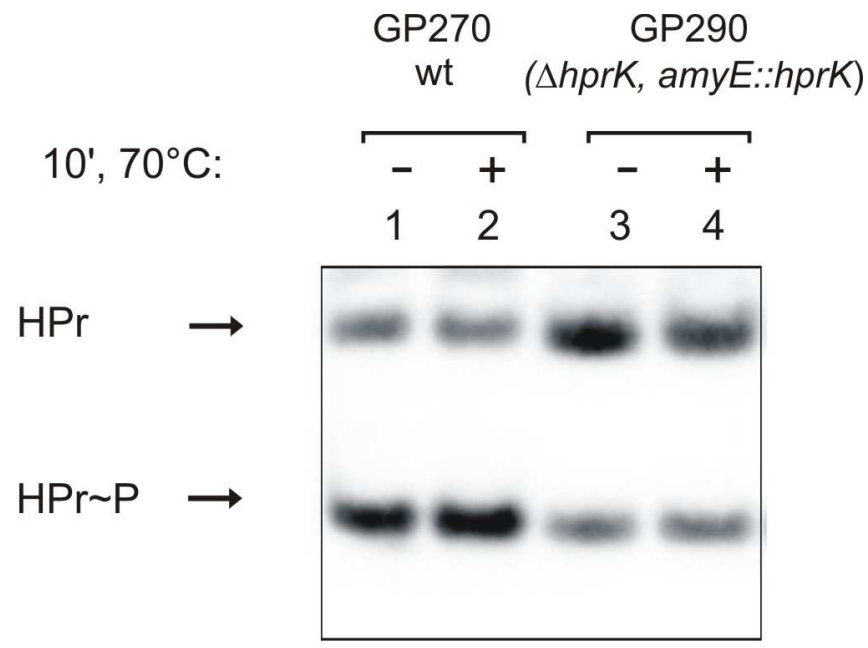

Figure. 18. Determination of the phosphorylation state of HPr in strains GP270 and GP290. Cells were grown in CSE + glucose and harvested at logarithmic phase as described before (Singh et $a l ., 2008) .1 \mu \mathrm{g}$ of total cell protein, each was separated on a $10 \%$ native PAA gel and HPr was detected by immuno-blotting in a western blot analysis. To discriminate between $\operatorname{HPr}(\operatorname{Ser}-\mathrm{P})$ and $\mathrm{HPr}\left(\right.$ His-P), an aliquot of each cell extract was heated at $70^{\circ} \mathrm{C}$ prior to loading (even numbered lanes). Histidine phosphorylation is heat labile and thus the two singly phosphorylated forms of HPr can be easily distinguished. 


\section{Formation of $\mathrm{HPr}(\mathrm{Ser}-\mathrm{P})$ is impaired in the mutant expressing $h p r K$ from an ectopic site} HPrK/P triggers CCR by phosphorylating the corepressors of CcpA (HPr and Crh). One possible explanation for the weak CCR by certain carbohydrates in the [AhprK amyE: hprK] mutant could be that the ectopically expressed HPrK/P is less active as a kinase as compared to its counterpart in the wild-type strain. In order to check this possibility, the phosphorylation state of HPr was assayed. For this purpose, cells were grown in CSE + glucose and protein extracts were separated on a native PAA-gel followed by Western blotting using antiserum directed against HPr (See Materials and Methods and Singh et al., 2008 for details on the procedure). In agreement with a recent publication (Singh et al., 2008), $56 \%$ of the HPr molecules were phosphorylated at Ser-46 (Fig. 18, compare lane 1 and 2). In contrast, the [ $\triangle$ hprK amyE: hprK] mutant, which expresses $h p r K$ ectopically, produced only $34 \%$ $\mathrm{HPr}($ Ser-P) (Fig. 18, compare lanes 3, 4 with lanes 1,2). This observation clearly establishes that a lower amount of $\operatorname{HPr}(\operatorname{Ser}-\mathrm{P})$ is produced in the $[\Delta h p r K$ amyE: hprK] mutant in comparison to the wild-type, explaining the weaker CCR.

\section{Is there a polar effect of the hprK::aphA3 mutation on genes located downstream in the hprK operon?}

Strain GP290 harbours the $h p r K$ gene in the amyE locus while most of the native $h p r K$ gene is deleted and replaced by the aphA3-kanamycin cassette (Fig. 19b) (Martin-Verstraete et al., 1999). The DNA fragment used to replace the $h p r K$ gene contains the aphA3 gene under control of its own promoter, which reads into the same direction as the authentic promoter of the $h p r K$ operon. In principle, it is conceivable that this arrangement generates polar effects on the expression of the genes located downstream in the hprK operon. This could account for the observed relief of CCR in strain GP290, provided that one of the genes downstream of $h p r K$ has a role in CCR. In principle, two different scenarios can be imagined:

1. The presence of the aphA3-promoter might generate a second transcript in addition to the transcript started at the authentic hprK promoter (Fig.19b). This could elevate the expression level of the genes encoded downstream of hprK.

2. The insertion of the aphA3 cassette yields naked stretches of mRNA, which are not covered by ribosomes, e.g. the 3' sequence of the hprK gene following the aphA3 resistance gene is not translatable. Non-translated RNAs are often rapidly degraded by RNases or may be subject to the formation of secondary structures that may block the translation of genes. Hence, this scenario would reduce expression of the downstream genes. 
In order to explore whether the presence of the aphA3 cassette affected the transcription of the genes encoded downstream of $h p r K$, we performed a Northern analysis. The various strains were grown in CSE-medium supplemented with glucose and total RNA was isolated from cells grown to exponential phase and subsequently separated on a denaturating agarose gel and blotted onto a nylon membrane. Transcripts of the $h p r K$ operon were detected using an RNA probe directed against yvoE RNA. As expected, a single prominent signal of a size of $\sim 4 \mathrm{~kb}$ was detectable in the wild-type strain GP270 (Fig. 20, lane 1). This confirms, that the genes of the $h p r K$ operon are co-transcribed as a penta-cistronic mRNA from a single promoter located upstream of $h p r K$ (Fig. 19a). A transcript of similar length was also detectable in strains GP289 and GP290 carrying the hprK::aphA3 cassette (Fig.20, lanes 2 and 3). However, in these strains an additional transcript of $\sim 4.8 \mathrm{~kb}$ was also detectable. These results are in agreement with the scenario described in Fig. 19b, i.e. the aphA3 promoter as well as the authentic $h p r K$ promoter contributes both to transcription of the genes downstream of the aphA3 cassette. These results demonstrate that the genes lgt-yvoF are properly transcribed in the $h p r K:: a p h A 3$ mutant, although the overall transcript level appears to be somewhat higher in comparison to the wild-type strain GP270. In conclusion, the results are in favour with a stronger transcription of genes lgt-yvoF in the strains carrying the hprK::aphA3 cassette, although an aberrant translation of these genes cannot be excluded.

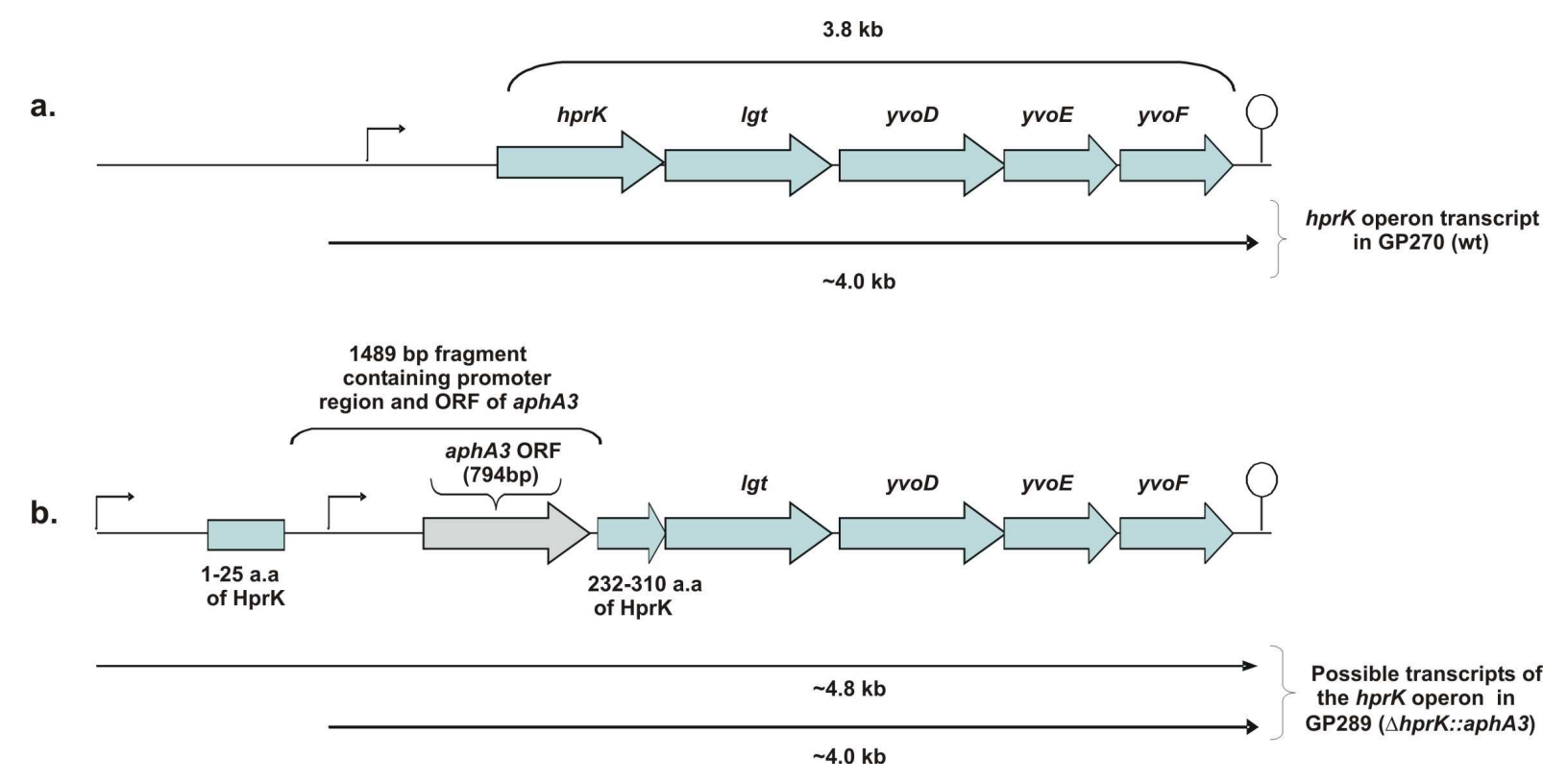

Figure. 19. Possible effects of the hprK::aphA3 allele on the expression of the genes downstream of $\boldsymbol{h p r K}$. (a) The length of the expected transcript in wild-type strain GP270 originating from the native promoter. (b) Due to the insertion of the aphA3 cassette, the length of the transcript originating from native promoter would increase by approximately 800 bp in strain GP290. An additional transcript is expected in strain GP290, originating from the promoter of the aphA3 cassette. 


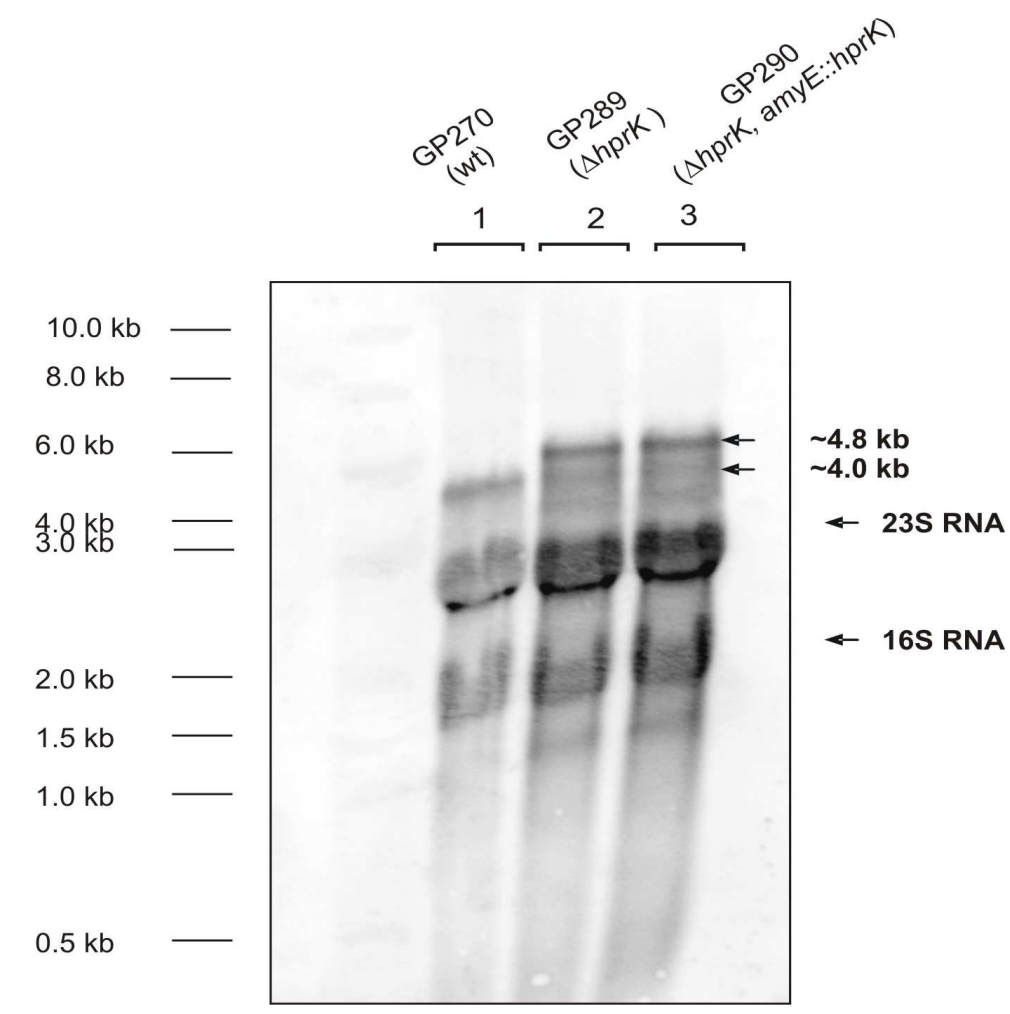

Figure. 20. Northern blot analysis of the hprK operon. Cells were grown in CSE minimal medium supplemented by $0.5 \%$ of glucose. Total RNA was separated by electrophoresis on a $0.8 \%$ agarose gel and then blotted onto nylon membrane followed by hybridization with a riboprobe specific for yvoE mRNA. The specific signals detected by the $y v o E$ riboprobe are marked by an arrow. $5 \mu \mathrm{g}$ of total RNA were loaded in each lane.

Deletion analysis of the $h p r K$ operon reveals that gene lgt is required for an undisturbed CCR

Our data raised the possibility that the disturbed CCR observed in the [AhprK amyE: hprK] strain, which expresses $h p r K$ ectopically, results from an altered expression level of the gene(s) downstream of the $h p r K:: a p h A 3$ cassette. This suggests, that one or more of these genes i.e. lgt, $y v o D, y v o E$ or $y v o F$ might have a role in CCR. In order to identify these gene(s), we constructed several deletion mutants lacking one or more of these genes. In these mutants, the corresponding genes were replaced by the aphA3 (kanamycin) resistance cassette, whereas the authentic $h p r K$ gene remained intact. These constructions resulted in strains GP851 ( $($ lgt, $\triangle y v o D, \Delta y v o E, \Delta y v o F)$, GP859 ( $\triangle y v o E, y v o F)$ and GP861 ( $\Delta y v o D$, $\Delta y v o E, \Delta y v o F$ ). Since a previous study suggested a role of YvoE in CCR (Mijakovic et al., 2002), we also constructed a $\triangle y v o E$ single mutant (strain GP852). Subsequently, the strains were grown on different carbon sources and the $\beta$-xylosidase (XynB) activities were determined (Table. 9). The strains GP852, GP859 and GP861 produced XynB activities very similar to the wild-type (Table. 9, compare column 1 with columns 3-5). Therefore, the 
absence of genes $\triangle y v o D, \triangle y v o E$ and $\triangle y v o F$ has no effect on CCR of XynB activity. In contrast, in strain GP851 lacking all four genes lgt, yvoD, yvoE and $y v o F$, a two- to three-fold relief from CCR was detectable, regardless of the carbon source included in the growth medium (Table 9, compare columns 1 and 2). The only difference between strains GP851 and GP861 is the additional lack of gene lgt in GP851. Hence, gene lgt is required for an undisturbed CCR, at least in a situation in which the three genes following lgt are also absent. Moreover, it can be concluded that the absence of the pyro-phosphatase YvoE has no impact on CCR, at least under the experimental conditions used in his work.

Table. 9. Catabolite repression of $\beta$-xylosidase by different carbon sources in various mutants. Standard deviations are shown in parentheses except for the measurements done once.

$\beta$-xylosidase activity in units/mg of protein

\begin{tabular}{cccccc}
\hline $\begin{array}{c}\text { Carbon } \\
\text { source }^{\mathrm{a}}\end{array}$ & $\begin{array}{c}\text { GP270 } \\
\text { wild-type }\end{array}$ & $\begin{array}{c}\text { GP851 } \\
\Delta l g t-y v o F\end{array}$ & $\begin{array}{c}\text { GP852 } \\
\Delta y v o E\end{array}$ & $\begin{array}{c}\text { GP859 } \\
\Delta y v o E-y v o F\end{array}$ & $\begin{array}{c}\text { GP861 } \\
\Delta y v o D-y v o F\end{array}$ \\
\hline Arabinose & $600(153)$ & $1292(13)$ & $598(20)$ & 660 & 684 \\
Maltose & $489(32)$ & $1153(669)$ & 570 & n.d & n.d \\
Gluconate & $243(76)$ & $454(128)$ & $324(70)$ & n.d & n.d \\
Sorbitol & $121(29)$ & $238(40)$ & 126 & n.d & n.d \\
Glycerol & $96(14)$ & $104(10)$ & $81(3)$ & n.d & n.d \\
Mannitol & $83(17)$ & 523 & 93 & 94 & 90 \\
Fructose & $66(6)$ & $75(8)$ & $74(22)$ & n.d & n.d \\
Glucose & $44(10)$ & $140(70)$ & $65(22)$ & $40(13)$ & $48(26)$ \\
\hline
\end{tabular}

a added to CSE medium (0.5\%), n.d = Not determined

\section{Discussion}

HPrK/P plays a key role for CCR in B. subtilis: It triggers the phosphorylation of HPr at Ser46 in response to the metabolic state in the cell. $\operatorname{HPr}(\operatorname{Ser}-\mathrm{P})$ serves as co-factor for the global transcriptional regulator $\mathrm{CcpA}$, which represses secondary catabolic genes in the presence of preferred substrates. HPrK/P is encoded by the first gene of a penta-cistronic operon. Little is known about the functions of the four genes co-transcribed with hprK. In this study, we provide evidence that at least one of these genes might also play a direct or indirect role in CCR. 
Initially, we observed that a deletion of the authentic hprK gene could not be complemented by $h p r K$ expression from an ectopic locus. The respective mutant exhibited a partial relief from CCR, when grown on glucose, fructose, mannitol or salicin. Interestingly, CCR by other carbon sources was not affected. Since the native $h p r K$ gene was replaced by an aphA3 antibiotic resistance cassette reading into the genes downstream, we speculated that a polar effect was responsible for the aberrant CCR in this strain. Indeed, Northern analysis demonstrated the presence of two transcripts: One started from the authentic hprK promoter and another one initiated from the foreign promoter preceding the antibiotic resistance cassette. As a result, the amounts of the lgt-yvoF transcript present in this mutant are higher than in the wild-type strain. These observations suggest that a higher expression level of one or more of the genes downstream of the resistance cassette was responsible for the partial loss of CCR.

To learn more about the putative roles of the proteins encoded in the $h p r K$ operon, we constructed mutants lacking one or more of the genes present downstream of $h p r K$ in the same operon. These experiments yielded several insights:

First, it turned out that the absence of $y v o E$ had no effect on CCR. Gene $y v o E$ encodes a protein exhibiting pyro-phosphatase activity in vitro (Mijakovic et al., 2002). HPrK/P uses $\mathrm{P}_{\mathrm{i}}$ as substrate for the dephosphorylation of $\mathrm{HPr}(\mathrm{Ser}-\mathrm{P})$ and thereby produces $\mathrm{PP}_{\mathrm{i}}$ as product. It was proposed that the so-generated pyro-phosphate is hydrolyzed by YvoE. Furthermore it was shown that in vitro $\mathrm{PP}_{\mathrm{i}}$ can substitute for ATP as substrate for the HPrK catalyzed phosphorylation of HPr. In addition, $\mathrm{P}_{\mathrm{i}}$ was reported to inhibit the kinase activity and to stimulate the phosphorylase activity of HPrK/P in vitro (Fieulaine et al., 2002; Mijakovic et $a l ., 2002)$. In this respect, a $y v o E$ mutation should generate, if there is any effect at all, a higher cellular level of $\mathrm{PP}_{\mathrm{i}}$ and perhaps less $\mathrm{P}_{\mathrm{i}}$. These conditions would stimulate the kinase activity of HPrK/P and thereby generate a stronger CCR. The absence of any effect of an yvoE mutation on CCR, as shown in this work, is may be due hydrolysis of PPi by other possible phosphatases, which can also utilize PPi, like YvoE.

Second, our results let us conclude that $y v o D$ and $y v o F$ have no essential function for CCR. The deletion of these genes had no detectable effect on CCR (Table 9). The putative functions of these genes remain obscure.

Finally, the data suggested that a functional lgt gene might be necessary for an undisturbed CCR: No effect on CCR could be detected in strains lacking the last three genes of the $h p r K$ operon. However, when $l g t$ was additionally deleted, a partial relief from CCR could be detected. We cannot rule out yet, that in addition to lgt one of the three downstream 
genes must be also absent to yield the observed phenotype. Future studies using a lgt single mutant should address this point and clarify whether absence of lgt is sufficient for the observed relief from CCR. In any case, the data demonstrate that presence of gene lgt is required for CCR, at least in the observed mutant background. Gene lgt encodes the prolipoprotein diacylglycerol transferase that catalyzes the first step in the lipomodification of bacterial lipoproteins (Leskelä et al., 1999). This modification anchors peripheral membrane proteins to the outer surface of the cytoplasmic membrane. There are three groups of lipoproteins known in Gram-positive bacteria. These include the substrate-binding domains of $\mathrm{ABC}$ transporters, proteins necessary for adhesion to various surfaces and proteins with enzymatic activity e.g. $\beta$-lactamases (Leskelä et al., 1999 and references therein). In E. coli lgt is an essential gene. In B. subtilis an lgt mutant is viable but disturbed in protein secretion and spore germination (Igarashi et al., 2004; Leskelä et al., 1999).In respect of all these findings, it is difficult to imagine how lgt could affect CCR. None of the transporters involved in the uptake of the carbohydrates tested in this study, is known to be a lipoprotein. Most likely, the lgt mutation affects CCR indirectly by the modification of the membrane composition and cell surface.

The phenotypes of the $\Delta l g t-y v o F$ mutant and the mutant expressing the ectopic hprK copy were dissimilar. The $\Delta l g t-y v o F$ mutant exhibited a partial relief from CCR ( 2- to 4fold) on all carbon sources. In contrast, in the ectopically complemented $h p r K$ mutant there was a much stronger relief from CCR, i.e. 5-14-fold, but only on a certain subset on carbohydrates: All these substrates, i.e. glucose, fructose, mannitol and salicin are transported by the PTS. On Non-PTS-substrates, CCR was unaffected. These different phenotypes make it rather unlikely that a diminished synthesis of genes $\lg t-y v o F$ is responsible for this selective relief from CCR. In contrast and in agreement with the Northern blot data, it appears conclusive that this phenotype is caused by the increased expression of genes lgt-yvoF, present downstream of the hprK::aphA3 cassette.

How can the selective relief from CCR in the ectopically complemented hprK mutant be explained? We made the observation that the level of $\mathrm{HPr}(\operatorname{Ser}-\mathrm{P})$, the active co-factor for CcpA, is significantly lower in this mutant in comparison to the wild-type. Hence, it is obvious, that the (de)phosphorylation step of HPr by HPrK/P is affected. Theoretically, it is conceivable that the over-expression of YvoE might affect CCR. YvoE removes pyrophosphate, which is also a substrate of HPrK kinase. This could lower HPrK kinase activity in the cell. However, such a mechanism should be operative independent of the carbon source utilized by the bacteria, which makes this explanation unlikely. An alternative 
explanation might take into account that all carbohydrates, on which CCR was impaired, are substrates of the PTS. Hence, it is conceivable that transport of these substrates is negatively affected, which would result in lower amounts of FBP, which is required for the allosteric activation of HPr kinase. Indeed, the ectopically complemented hprK mutant grows significantly slower on these PTS substrates in comparison to the wild-type, which is in support with this idea (our unpublished observations). Future transport studies may clarify this point. 


\section{Discussion}

\subsection{Role of PrpC in regulating the phosphorylation status of HPr}

So far, HPrK/P and EI were the only enzymes known to be involved in the (de)phosphorylation of HPr in Gram-positive bacteria. However, certain arguments suggest that there might be a need in B. subtilis for an additional protein that acts antagonistically to the kinase activity of HPrK/P. In vitro studies suggest that the ATP dependent kinase activity of B. subtilis HPrK/P is allosterically activated by FBP. In the presence of $10 \mathrm{mM}$ FBP and $5 \mathrm{mM}$ ATP, it can completely phosphorylate HPr in an 85 molar excess (Jault et al., 2000). B. subtilis cells grown in the presence of glucose contain $14 \mathrm{mM}$ FBP. However, 30-40\% of the total HPr remains unphosphorylated. The majority is phosphorylated at Ser-46 (Monedero et al., 2001; Singh et al., 2008). The other substrate for HPrK/P, ATP, has also been found, in vivo, to be within the range of in vitro experiments performed. During the uptake of glucose by L. lactis, the concentration of ATP is about $8 \mathrm{mM}$ (Neves et al., 1999). Hence, there is a contradiction between the in vivo and the in vitro data. Having HPr completely phosphorylated at serine residue is not advantageous for the cell. It would inhibit further PTS-mediated uptake of glucose because no histidine phosphorylated HPr is available for phospho transfer (Monedero et al., 2001). Thus, there should be a mechanism to achieve limit phosphorylation of HPr at its serine residue even in the presence of the most preferred carbon source which is glucose.

There are several possibilities that could explain the in vivo pattern of $\mathrm{HPr}(\mathrm{Ser}-\mathrm{P})$ in the presence of glucose: a) The intracellular ratio of HPrK/P to the HPr molecules is much lower in comparison to the protein concentrations used in in vitro experiments. Therefore, only 60$70 \%$ of HPr can be phosphorylated at Ser-46. This possibility appears to be less likely because artificially increased intracellular amounts of HPrK/P protein did not alter the phosphorylation pattern of HPr, when the cells grew on glucose (see supplementary material, Fig. 23) b) A so far unidentified phosphatase may counteract the effect of phosphorylation achieved by HPrK/P.

Recently, Halbedel, et al demonstrated that in M. pneumoniae PrpC dephosphorylates Ser-46 phosphorylated HPr. M. pneumoniae is classified as a Gram-positive bacterium belonging to the genus Mollicutes, which possesses the smallest genome among bacteria. Interestingly, HPrK/P and PrpC are among the few regulatory proteins which M. pneumoniae retained (Himmelreich et al., 1996). This prompted me to analyse the role of PrpC in regulating the phosphorylation state of HPr in B. subtilis. Indeed, in an in vitro 
dephosphorylation reaction, PrpC could dephosphorylate Ser-46 phosphorylated HPr (Singh et al., 2007).

PrpC of B. subtilis belongs to the PPM family of phosphatases and is a $\mathrm{Mn}^{+2}$ ion dependent Ser/Thr phosphatase (Obuchowski et al., 2000). The members of PPM family are widely distributed and are represented by protein phosphatase $2 \mathrm{C}$ from humans, Arabidopsis thaliana and bovine pyruvate dehyrogenase phosphatase in eukaryotes (Das et al., 1996; Meyer et al., 1994; Vassylyev and Symersky, 2007). In prokaryotes, most of the available knowledge regarding the distribution of PPM phosphatases comes from protein sequence and conserved catalytic domain similarity searches. Only a subset of these phosphatases have been investigated experimentally (Shi et al., 1998). In bacteria, PPMs have been most intensively studied in B. subtilis. They include SpoIIE, RsbU, RsbX and PrpC. All these proteins exhibit divalent metal ion stimulated protein phosphatase activity in vitro (Adler et al., 1997; Duncan et al., 1995; Yang et al., 1996). SpoIIE is involved in a signal transduction cascade that targets a sigma factor, $\sigma^{\mathrm{F}}$ involved in activating the transcription of stress response proteins (Duncan $e t$ al., 1995). RsbX and RsbU, constitute portions of a pathway that regulates the activity of sigma B. PrpC was shown to dephosphorylate elongation factor-G and PrkC, a membrane linked protein kinase, on their threonine residue in B. subtilis (Gaidenko et al., 2002; Obuchowski et al., 2000). PPM phosphatases can use a broad spectrum of phosphorylated substrates including the artificial substrate $p$-nitro phenyl phosphate (pNPP).

The biochemical properties of PrpC made it a suitable candidate for testing the possibility of being the phosphatase, acting against the HPrK/P kinase. Dephosphorylation of $\mathrm{HPr}(\mathrm{Ser}-\mathrm{P})$ in vitro is indeed catalysed by $\operatorname{PrpC}$ as shown for the proteins from B. subtilis and M. pneumoniae. This reaction was inhibited by $\mathrm{P}_{\mathrm{i}}$ (Halbedel et al., 2006; Singh et al., 2007). Dephosphorylation of $\operatorname{HPr}(\operatorname{Ser}-\mathrm{P})$ by B. subtilis PrpC could be completely inhibited by $10 \mathrm{mM}$ phosphate (Singh et al., 2007). In the presence of a rapidly metabolizable carbon source like glucose, intracellular phosphate levels are much lower compared to the cells grown on a poor carbon source (Mason et al., 1981; Thompson and Torchia, 1984). During the uptake of a rapidly metabolizable carbon source such as glucose by L. lactis, the concentration of $\mathrm{P}_{\mathrm{i}}$ are about $5 \mathrm{mM}$. In contrast, in the absence of a rapidly metabolizable carbon source, the concentration of $\mathrm{P}_{\mathrm{i}}$ increases to about $50 \mathrm{mM}$ (Neves et al., 1999). However, no difference was observed in the phosphorylation pattern of HPr in a $\triangle p r p C$ mutant as compared to wild type cells, grown in the presence of glucose (see supplementary material, Fig. 24). Thus it appears unlikely, that PrpC affect the in vivo phosphorylation state of the HPr, at least in the presence of good carbon source like glucose. This observation is very different from the situation in $M$. 
pneumoniae, where a prpC mutation drastically increases the intracellular $\mathrm{HPr}(\operatorname{Ser}-\mathrm{P})$, in the presence of glucose and glycerol. In M. pneumoniae wild type cells, $\mathrm{HPr}(\mathrm{Ser}-\mathrm{P})$ is present in considerable amounts in the presence of glycerol and glucose (Halbedel et al., 2006).

Interestingly, when the cells were grown in the presence of succinate (CSE medium) as the sole carbon source, the $\triangle p r p C$ strain exhibited an increased phosphorylation of HPr as compared to the wild type strain (30\% increase in the signal intensity). Succinate is a poor carbon source, and generates only $13 \%$ of $\operatorname{HPr}(\operatorname{Ser}-\mathrm{P})$ in the wild type cells (Singh et al., 2008). Succinate does not allow exertion of CCR of the reporter system used in this study $(x y n B)$. In a $\triangle p r p C$ mutant the $\operatorname{HPr}($ Ser-P) levels increased to $16 \%$ of the total HPr. However, these increased $\operatorname{HPr}(\operatorname{Ser}-\mathrm{P})$ levels did not lead to stronger repression of xynB. Probably a minor increase in $\mathrm{HPr}(\mathrm{Ser}-\mathrm{P})$ in $\operatorname{prpC}$ mutant does not lead to formation of the complex with CcpA, which is sufficient to repress the expression of the reporter system used here. This is in agreement with our finding that atleast $20 \%$ of intracellular $\mathrm{HPr}(\mathrm{Ser}-\mathrm{P})$ is required to exert 3 fold repression (Singh et al., 2008). In this respect, it would be interesting to analyze the CCR of the catabolic operons that are repressed in the presence of succinate. For e.g, the expression of the citM, which encodes the $\mathrm{Mg}^{+}$-citrate transporter, is known to be repressed in CSE medium (Warner et al., 2000). In previous studies, PrpC has been implicated in controlling a number of stationary phase processes (Gaidenko et al., 2002). In terms of the metabolic state of the cell, stationary phase is associated with nutrient limitation and an increasing intracellular phosphate concentration. This is in good agreement with the idea that PrpC functions under conditions poor carbon supply.

Crh, an HPr paralog in B. subtilis has been implicated in catabolite repression of some catabolic operons, when cells are grown in succinate or when cells enter stationary phase (Inacio and de Sa-Nogueira, 2007; Warner et al., 2003). Crh can be phosphorylated at Ser-46 residue and thus it is tempting to speculate that $\operatorname{PrpC}$ may be involved in the (de)phosphorylation of Crh, especially when Crh-specific functions are required.

Generically, HPrK/P from M. pneumoniae has the kinase activity as its preferential mode of action. In comparison, HprK/P from B. subtilis requires 300-fold molar excess of ATP and stimulation by FBP to act as a kinase under otherwise similar conditions. Therefore, the requirement for an alternate phosphatase could be a property of the organisms bearing HPrK/Ps with low HPr(Ser-P) dephosphorylation activity. Thus, PrpC in M. pneumoniae, may be more important in effecting the phosphorylation state of $\operatorname{HPr}(\operatorname{Ser}-\mathrm{P})$ and regulating the carbon metabolism, in contrast to the PrpC in B. subtilis. Interestingly, the residues involved in phosphate binding, as suggested by the crystal structure of human PP2C proteins, are 
perfectly conserved in M. pneumoniae and B. subtilis PrpC proteins (Halbedel et al., 2006, Obuchowski et al., 2000). Thus, the differences in acting as a phosphatase on HPr(Ser-P) in vivo, from these two organisms might reside in the subtle differences in the structure of HPr proteins.

Moreover, as evident from the findings from this work, PrpC can not be the answer to the presence of unphosphorylated HPr in B. subtilis under conditions of good carbon supply.

\subsection{Hierarchical nature of catabolite repression exerted by various carbon sources}

Much effort has been devoted to understand the mechanisms underlying catabolite repression in B. subtilis. Various regulatory mechanisms have been elucidated, among which global repression achieved by CcpA is the most important one (see introduction). The rearrangements in CcpA secondary structure, induced by $\mathrm{HPr}(\mathrm{Ser}-\mathrm{P})$ and $\mathrm{Crh}(\mathrm{Ser}-\mathrm{P})$ binding, are a prerequisite for adapting the DNA binding conformation and consequently for transcription regulation. Intensive mutagenesis studies, and in particular, the determination of the structure of the $\mathrm{CcpA} / \mathrm{HPr}(\mathrm{Ser}-\mathrm{P})$-cre ternary complex, have contributed considerably to the understanding of CcpA function in CCR in Gram-positive bacteria (Kraus et al., 1998; Kraus and Hillen, 1997; Küster-Schock et al., 1999; Schumacher et al., 2004). In this work, a systematic analysis of the global catabolite repression pathway is presented in which a variety of different of carbon sources were compared. Use of chemically different sugars provides the opportunity to evaluate every step involved in mediating catabolite repression, including uptake and metabolism of different carbon sources. In E. coli, the transport of a carbohydrate via the PTS is sufficient to elicit CCR (Deutscher, 2006). In contrast, in B. subtilis, metabolism of a repressing sugar has been found to be required for CCR (Nihashi and Fujita, 1984).

Repression of the expression of the $x y n P B$ operon provides an efficient reporter system for this study. The $x y n P B$ operon encodes for the xyloside transporter (XynP) and $\beta$ xylosidase (XynB). The metabolism of xylosides generates xylose, which is the inducer for the expression of the $x y n P B$ operon (Lindner et al., 1994; Galinier et al., 1997). $\beta$-xylosidase activity can be measured in a colorimetric enzymatic assay. The promoter region of this operon bears two cis-acting elements: an operator site recognised by an operon specific repressor, XylR and a cre sequence, separated by 200bp (see Fig. 21). Xylose, as an inducer for this system, releases XylR from its operator site. XylR is also known to act as an anti inducer in the presence of high intracellular concentration of G6P (Dahl et al., 1995). Thus, in 
a mutant strain lacking a functional $\mathrm{XylR}$, only catabolite repression via $\mathrm{CcpA} /$ cre is operative (Kraus et al., 1994). Moreover, use of a XylR mutant avoids the addition of xylose in the media. The presence of only a single cre site, further simplifies the situation. The Regulation of $x y n P B$ operon via CcpA is strongly regulated and the expression can be repressed by as much as 36 folds in the presence of glucose (Singh et al., 2008). Additionally, as demonstrated in this work, a number of additional carbon sources also repressed the $x y n P B$ expression, making it an optimal reporter system. Catabolic operons like iol and gnt are not repressed in the presence of carbon sources like ribose and arabinose, thus limiting the use of sugars, categorized here as poor carbon sources, to be studied (Nihashi and Fujita, 1984).

The carbon sources used in this study include hexoses (glucose and fructose), pentoses (arabinose and ribose), sugar alcohols (glycerol and sorbitol), oligo- $\beta$-glucosides (salicin) and disaccharides (sucrose and maltose) and sugar acids (gluconate). They comprise both, PTS and non PTS carbon sources (see Fig. 2). The various carbon sources repressed $x y n B$ expression, to different degrees. In general, glucose and fructose (36- and 25-folds respectively) are the strongest repressing carbon sources, followed by mannitol, sorbitol, glycerol, salicin, sucrose and gluconate (20- to 7-folds). The weakest repression was exerted by maltose, arabinose and ribose (2- to 3 -folds). No repression occurs when only succinate is included in the media (CSE medium). Additionally, the absence of CcpA or of its known corepressor, HPr and Crh, completely abolished the repressing potential of almost every carbon source. This demonstrated that repression by various carbon sources is solely dependent on the CcpA mediated CCR pathway. Interestingly, the presence of sorbitol elicited a residual 34 fold repression in the $\triangle c c p A$ background. A deviating behavior for sorbitol, has been observed before. The expression of the gltAB operon in $B$. subtilis, encoding for the enzyme glutamate synthase, is induced in the presence of sorbitol. This induction is independent of CcpA and is impeded in an EI mutant, which can not accept phosphate from PEP (ptsI H189A). In B. subtilis, sorbitol uptake is mediated by a Sorbitol $/ \mathrm{H}^{+}$symporter (GutA) and is independent of the PTS activity (Chalumeau et al., 1978). A possible phospho transfer event from EI to a potential activator of gltAB operon was suggested to explain this observation (Schmalisch, 2004). However, in an EI mutant strain, XynB activity in the presence of sorbitol is not altered. Obviously, no mechanism parallel to that observed for the induction of glt $A B$ operon by sorbitol prevails for $x y n P B$ operon. Indeed, this sorbitol induced, CcpA independent repression mechanism seems to be specific for the $x y n P B$ operon as the repression of the RocG was completely relieved in a ccpA mutant (Singh et al., 2008). It could 
also be established in this work that sorbitol exerts this residual repression at the level of the transcription of the operon.
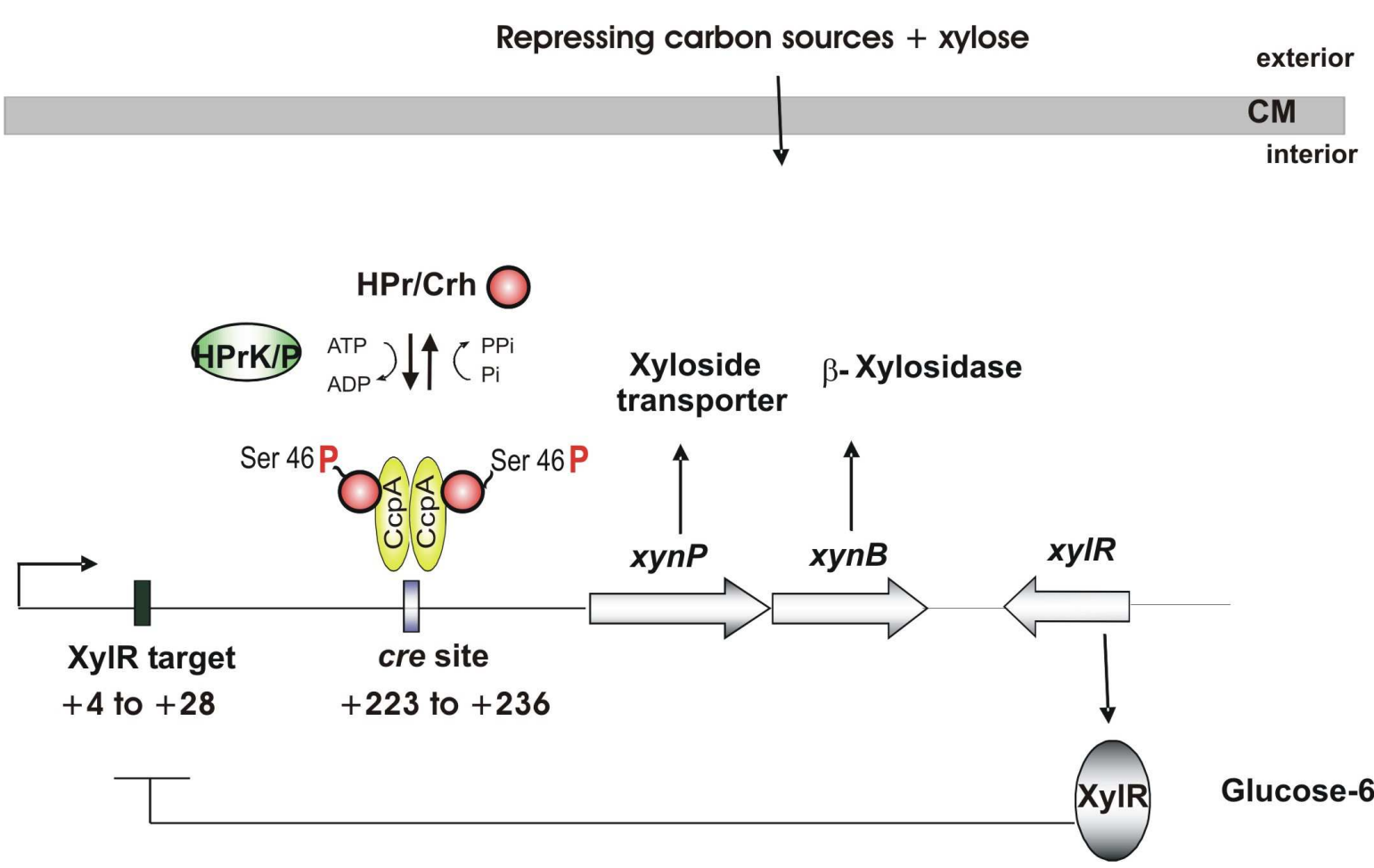

Glucose-6-P

Figure. 21. Organisation and regulation of the $x y n P B$ operon in B. subtilis. The operon is under control of dual regulation. In the absence of the inducer xylose, the operon specific repressor XylR binds to the XylR target site, present $3 \mathrm{bp}$ downstream of the transcription start site (+1). A 14bp cre site recognised by CcpA is present downstream of the XylR target site. In the presence of a repressing carbon source, the CcpA/co-repressor complex is formed and represses the expression of the operon by binding to the cre site. In addition, G6P acts as an anti-inducer by binding to XylR and thereby opposing the inducing effect of xylose. This leads to an additional repression in the presence of glucose.

\subsection{HPr(Ser-P) levels in the cell govern the strength of CcpA-medited catabolite repression by various carbon sources}

The hierarchical nature of repression observed in the presence of different carbon sources suggested that the repressor complex, $\mathrm{CcpA} / \mathrm{co}-$ repressor is formed to different extents. Since the intracellular levels of CcpA as well as of its cofactors remain constant, irrespective of the carbon source used (this work), it is conceivable that the extent of repressor complexes formed inside the cell is a function of phosphorylation of HPr and Crh. Previous investigations established that $\operatorname{HPr}(\operatorname{Ser}-\mathrm{P})$ and $\operatorname{Crh}(\operatorname{Ser}-\mathrm{P})$ do not act synergistically in allowing the binding of CcpA to the $x y n P B$ cre site (Galinier et al., 1999). Infact, in vivo, HPr 
seems to efficiently take over the function of being a co-repressor, in a crh mutant strain. Similar observations have been made for other catabolic genes subjected to CcpA regulated repression (Inacio et al., 2003; Martin-Verstraete et al., 1999). This clearly indicates that the CcpA mediated repression is not an additive function of the two co-repressors.

On the other hand, in a strain which expresses a ptsHl allele, coding for an HPr variant with non phosphorylatable alanine rather than a serine, a clearly different picture was observed. In such a mutant, the repression was relieved 3-8 fold in the presence of glucose, fructose and mannitol, but remained similar to a wild type strain in the presence of other carbon sources. These carbon sources exert the strongest repression. The HPrS46A variant is competent in PTS mediated sugar uptake (Deutscher et al., 1994; Reizer et al., 1989; Ye and Saier, Jr., 1996). Thus, impaired uptake of glucose, fructose and mannitol can not explain the weaker repression in this strain. Similarly, salicin and sucrose, which are also PTS substrates, when present in the media could elicit repression similar to the wild type strain (this work).

An alternative explanation can be envisioned as follows. In the presence of sorbitol and glycerol, Crh is capable of exerting 20 fold repression. It has been shown that as compared to $\mathrm{HPr}$, Crh is synthesized in 100-fold lower amounts in the presence of glucose (Görke et al., 2004). Apparently, this low concentration of a CcpA co-repressor is sufficient to carry out a 20 -fold repression of the $x y n P B$ operon. For achieving a stronger repression a higher concentration of co-repressor would be required. Furthermore, it was shown for the $B$. subtilis $\mathrm{CcpA} / \mathrm{Crh}(\operatorname{Ser}-\mathrm{P})$ complex that its binding to the cre site in vitro is not stimulated by metabolites like FBP and G6P (Schumacher et al., 2007; Seidel et al., 2005). Thus the repression observed in a $\mathrm{ptsH} 1$ mutant strain is independent of the allosteric stimulation of the $\mathrm{CcpA} / \mathrm{Crh}(\mathrm{Ser}-\mathrm{P})$ complex by metabolites. Taken together, these findings suggest that a higher concentration of co-repressor and the allosteric activation of the CcpA complex by metabolites are required to achieve very strong repression.

Collectively, these findings clearly establish that the role of Crh in CCR only becomes prevalent in the absence of HPr. In addition, numerous B. subtilis operons are completely relieved from $\mathrm{CCR}$ in a ptsHl mutant, suggesting that their cre site might not be recognised by $\mathrm{CcpA} / \mathrm{Crh}(\operatorname{Ser}-\mathrm{P})$ (Deutscher et al., 1994). In summary, HPr is the more relevant corepressor of CcpA. Gene, crh has been only detected in Bacilli. The genomes of all other Gram-positive bacteria lack a crh gene. Thus, $\operatorname{HPr}(\operatorname{Ser}-\mathrm{P})$ seems to be sufficient to mediate CCR in Gram-positive organisms, independently of whether they possess Crh or not (Deutscher et al., 2006). Although, crh can partially complement the function of HPr, the primary function might be unrelated to CCR. For instance, it was demonstrated that 
unphosphorylated Crh can inhibit the enzymatic activity of glyceraldehyde-3-phosphate dehydrogenase (GapA) (Pompeo et al., 2007).

Most of the stronger repressing carbon sources were found to be PTS substrates. Presence of a PTS substrate stimulates the transcription of HPr, leading to a 3 fold increased amount in the cell as judged from the Lac-Z fusion data (Görke et al., 2004; Stülke et al., 1997). Moreover, western blot analysis suggested similar amounts of HPr regardless of the carbon source used (Singh et al., 2008). Therefore, the differences in repression are not caused by different amounts of HPr present in the cell. Since, HPr can not bind CcpA unless its serine 46 residue is phosphorylated (Jones et al., 1997), the intracellular HPr(Ser-P) rather than total HPr should decide the extent of repression. Indeed, large amounts of HPr(Ser-P) are formed in the presence of strongly repressing carbon sources such as glucose. In contrast, negligible amounts of $\mathrm{HPr}(\mathrm{Ser}-\mathrm{P})$ are present when cells grew on CSE medium with succinate (Ludwig et al., 2002; Monedero et al., 2001). High amounts of intracellular HPr(Ser-P) could also be observed in the presence of other strongly repressing carbon sources such as fructose, sorbitol, glycerol, salicin, mannitol and sucrose. In the presence of these carbon sources, 50$70 \%$ of all HPr molecules are phosphorylated at serine residue. When the cells were grown on sugars that cause weaker repression, the $\mathrm{HPr}(\operatorname{Ser}-\mathrm{P})$ amounts varied from $13-32 \%$, being lowest in the presence of succinate (CSE medium) and highest in the presence of gluconate (see Fig. 22). An analogous situation exists in E. coli, for which several carbon sources are suggested to cause CCR. This has been attributed to the ability of these carbon sources to affect the phosphorylation state of the master regulator of CCR, EIIA ${ }^{\text {glu }}$ (Bettenbrock et al., 2007; Hogema et al., 1998). In E. coli, a correlation between the growth rate, the $\mathrm{PEP} /$ pyruvate ratio and the amount of dephosphorylated EIIA ${ }^{\text {glu }}$ was demonstrated. Carbon sources, which support fast growth, regardless of being a substrate of the PTS, exert CCR. The fast growth on carbon sources reflects a high flux through glycolysis which is accompanied by a low PEP/pyruvate ratio in the cell. This can cause EIIA ${ }^{\text {glu }}$ to be present in the unphosphorylated state, because the concentration of phosphate donor of the PTS, decreases in the cell (Hogema et al., 1997; Hogema et al., 1998). Owing to this, even pentoses such as arabinose can exert CCR in E. coli (Bettenbrock et al., 2007). On the other hand, arabinose also supports fast growth of $B$. subtilis cells, $(\mu=0.98)$ but, exerts only 2 fold repression of the XynB activity. In general, in B. subtilis there is no strict correlation between the growth and the strength of repression exerted, by a given carbon source. Except for ribose and succinate on which growth was significantly slower, all other carbon sources allowed fast 
growth of B. subtilis (specific growth rate ( $\mu$ ) of 0.83-0.98). In conclusion, HPr(Ser-P) levels in the cell can determine the hierarchy of the repression exerted by various carbon sources.

\subsection{Modulation of the phosphorylation state of HPr by the nature of the carbon source}

In this work, the repressing potential of various carbon sources is evaluated by using the $x y n P B$ operon as the reporter system. Using this system a correlation between the extent of the CcpA-mediated repression of the $x y n P B$ operon and the intracellular $\operatorname{HPr}(\operatorname{Ser}-\mathrm{P})$ is demonstrated (see Table. 4, chapter 3). Interestingly, not only the $\operatorname{HPr}(\operatorname{Ser}-\mathrm{P})$ levels varied depending on the carbon source, but also the HPr(His-P) levels showed variation. Most of the stronger repressing carbon sources are PTS substrates. These substrates generated $50-70 \%$ of intracellular $\mathrm{HPr}(\mathrm{Ser}-\mathrm{P})$. Moreover, $\mathrm{HPr}(\mathrm{His}-\mathrm{P})$ is undetectable on these substrates and 40$50 \%$ of total HPr in vivo is present in the unphosphorylated form. It has been reported that glucose grown $S$. salivarius cells also contain only a small amount of $\mathrm{HPr}(\mathrm{His}-\mathrm{P})$ (Thevenot et al., 1995). Presence of a PTS substrate drains away the $P_{i}$ from the general PTS proteins, partly explaining the detection of unphosphorylated form of $\mathrm{HPr}$ and low amounts of HPr(His-P). This low amount of HPr(His-P) seems to be sufficient to allow the continued growth on a given PTS substrate.

Another interesting role of $\operatorname{HPr}(\operatorname{Ser}-\mathrm{P})$ is in autoregulation of various catabolic operons. In B. subtilis, glpFK operon is repressed in the presence of glucose via two independent mechanisms: an antiterminator-dependent regulation and a $\mathrm{CcpA} /$ cre mediated repression. In the presence of constitutively active glycerol kinase, the expression of glpFK operon is always high, irrespective of the presence of the substrate glycerol in the media (Darbon et al., 2002). Presence of glucose in such a situation mediates 3- to 4-fold CcpA dependent repression. Surprisingly, a similar level of repression could also be observed when glycerol was included in media (Darbon et al., 2002). The repression was lost in a ccpA mutant, suggesting that glycerol can to some extent regulates its own uptake, via HPr(Ser-P). Similarly, gutAB operon encoding for glucitol permease and glucitol dehydrogenase, is induced in the presence of sorbitol (Chalumeau et al., 1978). Deleting the negative regulatory cre site increased the induced levels of sorbitol dehydrogenase (Ye and Wong, 1994). These observations point to an autoregulatory mechanism of carbohydrate utilization in which the rate of carbohydrate uptake and metabolism is adapted to the metabolic state of the cell, i.e the intracellular concentration of glycolytic intermediates. 
Table. 10. The cre sequences present in the regulatory region of various catabolic operons dedicated for the metabolism of the carbon sources used in this study.

\begin{tabular}{|c|c|c|c|c|}
\hline Gene/operon & Function & cre sequence§ & Position\# & Reference* \\
\hline \multirow{2}{*}{ Consensus cre } & & Half site $\downarrow$ Half site & & \multirow[b]{2}{*}{$1,2,3$} \\
\hline & & $\begin{array}{l}\text { TGWAARCGYTWNCA } \\
1234567891011121314\end{array}$ & & \\
\hline xynPB cre & Xylose metabolism & TGAAAGCGCTTTTA & D & 4 \\
\hline rbs cre & Ribose metabolism & TGTAAACGGTTACA & $\mathrm{O}$ & 5 \\
\hline araA cre & Arabinose metabolism & TGGAAACGGTTACA & D & 6 \\
\hline araE cre & Arabinose metabolism & TGAAAGCGTTTTAT & D & 6 \\
\hline gntRup cre & Gluconate metabolism & TGAAAGTGTTGCAT & $\mathrm{O}$ & 7 \\
\hline gntRdown cre & Gluconate metabolism & TGAAAGCGGTACCA & D & 7 \\
\hline bgIPH cre & Aryl- $\beta$-glucoside metabolism & TGAAAGCGTTGACA & $\mathrm{O}$ & 8 \\
\hline gutB cre & Sorbitol metabolism & TGTATGCACTTACA & D & 9 \\
\hline glpFK cre & Glycerol metabolism & TGACACCGCTTTCA & $\mathrm{O}$ & 10 \\
\hline mtIA cre & Mannitol metabolism & TGTAAGCGTTTTAA & $\mathrm{O}$ & 11 \\
\hline$m t I D$ cre & Mannitol metabolism & TGTGAACGAAACGA & D & 11 \\
\hline lev cre & Fructose metabolism & TGAAAACGCTTaACA & $\mathrm{U}$ & 12 \\
\hline
\end{tabular}

. $\S-\mathrm{W}=\mathrm{A}$ or $\mathrm{T}, \mathrm{R}=\mathrm{A}$ or $\mathrm{G}, \mathrm{Y}=\mathrm{C}$ or $\mathrm{T}, \mathrm{N}=$ any base

\# - Position indicate the relative location of cre site with respect to the transcription start site or the promoter. $\mathrm{D}, \mathrm{O}, \mathrm{U}$ indicates localization the cre downstream, overlapping, and upstream of the promoter/transcription start site. Repression via cre site requires cre site to be present downstream or overlapping with the promoter region. This allows repression of transcription by blocking the initiation of transcription or preventing the transcription elongation by a roadblock mechanism. For levanase operon, the cre site is localised upstream of the promoter. However in this case, it overlaps with the binding site for the activator of the operon, LevR, thus mediating repression.

*- 1 (Weickert and Chambliss, 1990), 2 (Kim and Chambliss, 1997), 3 (Martin-Verstraete et al., 1995), 4 (Galinier et al., 1999) , 5 (Strauch, 1995) , 6 (Inacio et al., 2003), 7 (Miwa et al., 1997), 8 (Krüger et al., 1996) , 9 (Ye and Wong, 1994), 10 (Darbon et al., 2002), 11 (Watanabe et al., 2003), 12 (Galinier et al., 1999).

- The dyad is indicated by an arrow.

However, any catabolic operon must resist a complete self repression, so that the respective catabolic genes are turned on when required. In B. subtilis, CcpA mediated repression can be avoided by lacking a potential cre site. This is the case for the ptsGHI operon which encodes for EII ${ }^{\text {glu }}$ and the general PTS components HPr and EI (Stülke et al., 1997). This explains the exceptional position of glucose as a repressing sugar. It is plausible that autoregulation of glucose uptake is mediated by lowering the $\mathrm{HPr}(\mathrm{His}-\mathrm{P})$ amounts in the cell to the extent that is sufficient to support rapid growth, but prevents deleterious 
accumulation and uptake. This is evident by an earlier report where the uptake rate for glucose was diminished in wt $B$. subtilis cells in comparison to the strain bearing the ptsHI mutation, at high concentration of substrate (Ye and Saier, Jr., 1996).

However, all of the catabolic operons directly subjected to CcpA dependent catabolite repression, harbours a potential cre site. The CcpA dimer interacts with 7 bp of the $14 \mathrm{bp}$ cre site (as shown in Table. 10) and makes 25 phosphate contacts (Schumacher et al., 2004). As shown in Table. 10, cre sites not only show sequence deviations from the consensus sequence but also degeneracy in their location. The precise docking of the Helix turn Helix (HTH) motif of CcpA can differ, depending on the cre sequence. However, despite of this different docking, key base and phosphate contacts can be preserved (Schumacher et al., 2004). Therefore, CcpA is flexible enough to bind half sites with altered sequences. Considering this, it is possible that the cre site of a particular catabolic operon responds strongly to the $\mathrm{CcpA} / \mathrm{HPr}(\mathrm{Ser}-\mathrm{P})$ complex formed in the presence of a stronger repressing carbon source. However, it is not strongly repressed by the $\mathrm{HPr}(\mathrm{Ser}-\mathrm{P})$ amounts generated by the presence of the cognate substrate of the operon. This idea discussed above may explain how the self repression is avoided but hierarachy of repression is maintained by different carbon sources.

\subsection{Formation of doubly phosphorylated HPr in B. subtilis}

In the presence of weakly repressing carbon sources like succinate, ribose, maltose, arabinose, gluconate and glycerol, a considerable amount of $\operatorname{HPr}($ Ser-P)(His-P) was detectable. The peculiarity of this observation resides in the following facts: a) In B. subtilis and other Grampositive bacteria both phosphorylation events are considered to be mutually exclusive b) The presence of $\mathrm{HPr}(\mathrm{Ser}-\mathrm{P})(\mathrm{His}-\mathrm{P})$, is almost always accompanied by the presence of considerable amounts of histidine phosphorylated HPr except for glycerol (see Fig. 22).

Doubly phosphorylated HPr can be a result of either phosphorylation of $\operatorname{HPr}(\operatorname{Ser}-\mathrm{P})$ by EI or phosphorylation of $\mathrm{HPr}($ His-P) by $\mathrm{HPrK} / \mathrm{P}$. For B. subtilis $\mathrm{HPr}$, a negative charge at serine 46 , renders it a poor substrate for EI catalysed phosphorylation in vitro $\left(\mathrm{K}_{\mathrm{M}}=8-10\right.$ fold higher than that of wild type HPr) (Reizer et al., 1992). Serine 46 of HPr faces the glutamate84 side chain of EI, as observed in the structure of the E. coli HPr-EI complex (Garrett et al., 1999). Thus, phosphorylation at Ser-46 is believed to result in a repulsion between the negatively charged carboxylate of Glu-84 and phosphate group of Ser-P causing a disturbance in the interaction of the two proteins (Garrett et al., 1999). This glutamate is also present in EI of B. subtilis (Kunst et al., 1997). 
Doubly phosphorylated HPr is also present in M. pneumoniae and oral Streptococci (Halbedel and Stülke, 2005; Thevenot et al., 1995). However, in M. pneumoniae EI, Glu-84 is replaced with a threonine, explaining the formation of $\operatorname{HPr}(\operatorname{Ser}-\mathrm{P})(\mathrm{His}-\mathrm{P})$ in this organism (Halbedel, 2006). Similarly, in S. salivarius, EI requires an acidic $\mathrm{pH}$ to accept $\mathrm{HPr}(\mathrm{Ser}-\mathrm{P})$ as a substrate in vitro. At acidic conditions, the negative charge of Glu-84 is neutralized to overcome the electrostatic repulsion of the serine phosphorylated HPr (Casabon et al., 2006). Interestingly, B. subtilis EI was also shown to utilize $\mathrm{HPr}(\operatorname{Ser}-\mathrm{P})$ as substrate, at slightly alkaline $\mathrm{pH}$ (Casabon et al., 2006). However, Bacilli unlike Streptococci maintain an intracellular pH near neutrality (Breeuwer et al., 1996). Thus, it is reasonable to assume that in B. subtilis HPr(Ser$\mathrm{P})($ His-P) is not a result of EI activity on $\mathrm{HPr}(\mathrm{Ser}-\mathrm{P})$. This assumption is further supported by the fact that in B. subtilis only a low level of intracellular $\operatorname{HPr}(\operatorname{Ser}-\mathrm{P})$ was present, when the doubly phosphorylated form was also detected. Theoretically, doubly phosphorylated HPr can be considered a result of EI dependent phosphorylation of $\mathrm{HPr}(\operatorname{Ser}-\mathrm{P})$ when $\mathrm{HPr}(\mathrm{Ser}-\mathrm{P})(\mathrm{His}-$ P) and $\operatorname{HPr}($ Ser-P) forms are present together in the cell. For instance, in S. mutans, the concentration of doubly phosphorylated HPr concomitantly increases with the HPr(Ser-P) concentration.

The alternative possibility that $\operatorname{HPr}(\operatorname{Ser}-\mathrm{P})($ His-P) is formed in vivo as a result of HPrK/P activity on $\mathrm{HPr}($ His-P), is also not in agreement with the available in vitro data. The phosphorylation of B. subtilis HPr by HPrK/P is shown to be inhibited when HPr was preincubated with PEP and EI. Additionally, HPrK/P exhibited a 10 times higher $\mathrm{K}_{\mathrm{M}}$ for $\mathrm{HPr}$ allele with a glutamate at position 15 rather than a histidine (Reizer et al., 1998). Since, the occurrence of $\operatorname{HPr}(\mathrm{Ser}-\mathrm{P})(\mathrm{His}-\mathrm{P})$ parallels the presence of $\mathrm{HPr}(\mathrm{His}-\mathrm{P})$ in the cell, it appears conceivable that $\mathrm{HPrK} / \mathrm{P}$ phosphorylates $\mathrm{HPr}(\mathrm{His}-\mathrm{P})$. In contrast to the in vitro observations, it seems that there are conditions in vivo which stimulate the formation of doubly phosphorylated HPr by HPrK/P. As mentioned before, double phosphorylation of HPr was prevalent in the presence of histidine phosphorylated HPr, irrespective of the presence of unphosphorylated HPr. This suggests that HPrK/P can specifically utilize HPr(His-P) as a substrate. Thus, it can be proposed that in the presence of large amounts of intracellular HPr(His-P), HPrK/P can display an altered specificity towards its substrate. This seems to be an interesting mode of regulation to limit the $\operatorname{HPr}(\operatorname{Ser}-\mathrm{P})$ amounts in the cell. In agreement with this idea, when $B$. subtilis cells grows in the presence of $0.1 \%$ glucose (as compared to $0.5 \%$, which was used in this work), doubly phosphorylated HPr start appearing. 


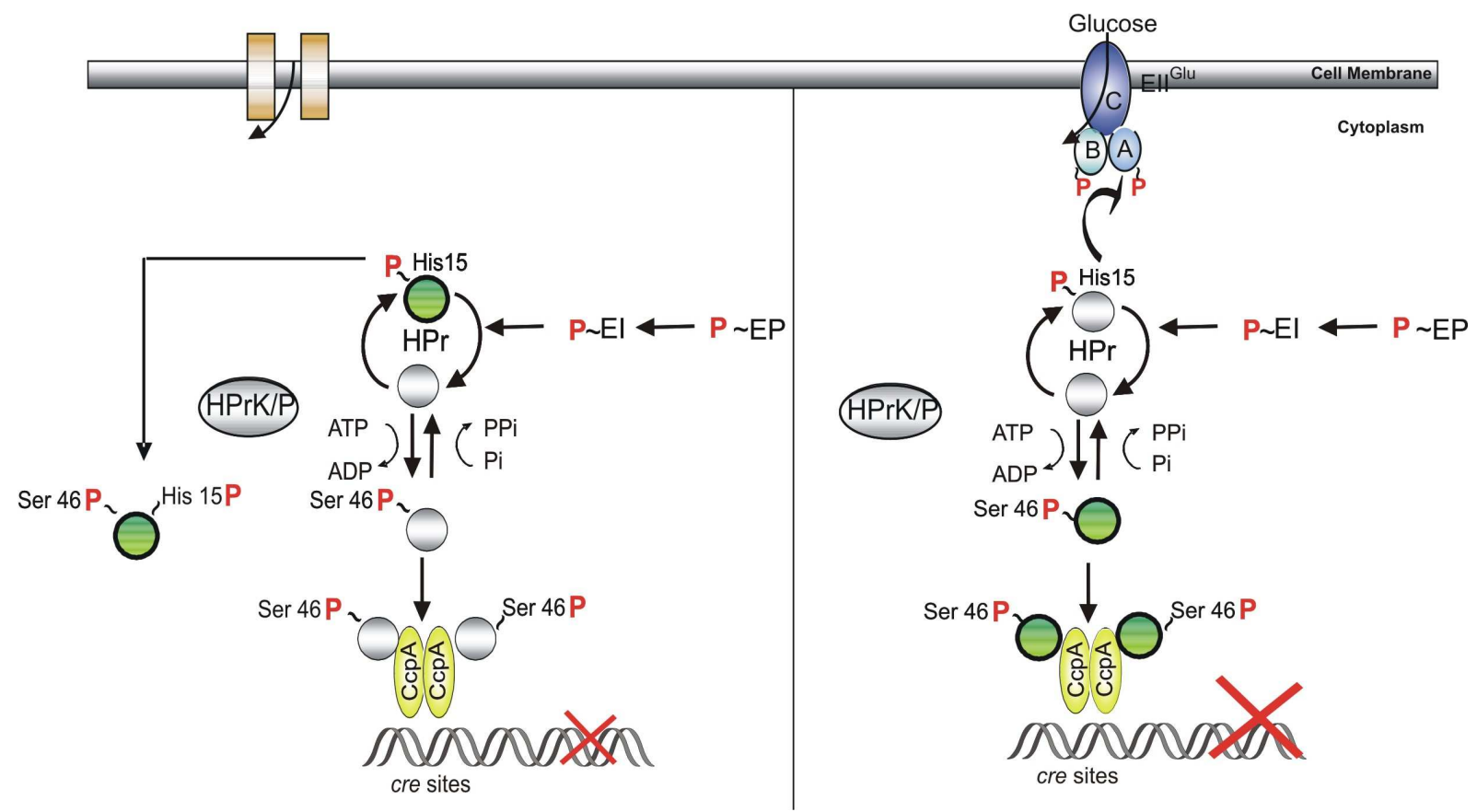

Figure. 22. The phosphorylation state of $\mathrm{HPr}$ in the presence of different carbon sources. The predominant forms of HPr are highlighted by a green colour. Most of the weakly repressing carbon sources are non-PTS carbon sources, except for maltose. In the presence of such carbon sources, the phosphate received by HPr via EI and PEP, is retained on HPr, resulting into high intracellular $\mathrm{HPr}($ His-P). Low HPrK/P activity in the presence of these carbon sources, results in lower $\mathrm{HPr}(\mathrm{Ser}-\mathrm{P})$ in the cells. Moreover, HPrK/P probably exhibits an altered substrate specificity in the presence of high intracellular $\operatorname{HPr}($ His-P) and can generate doubly phosphorylated form. In the presence of strongly repressing carbon sources, $\mathrm{HPr}(\mathrm{Ser}-\mathrm{P})$ is the predominant form. The remaining HPr is present in unphosphorylated form. Since strongly repressing carbon sources are mostly PTS substrates, the phosphate from the $\mathrm{HPr}(\mathrm{His}-\mathrm{P})$ is directed towards the incoming carbon source. Glucose is shown as an example for strongly repressing carbon source.

On the other hand, the amount of unphosphorylated HPr remained similar (Schmalisch, 2004). Obviously, the presence of a lower concentration in the medium increases the phosphorylation of HPr at its histidine residue.

In $M$. pneumoniae, and oral Streptococci, doubly phosphorylated HPr is detected in the presence of a rapidly metabolized PTS sugar for e.g glucose. It is possible that doubly phosphorylated HPr transfers phosphate towards the EIIs (Halbedel and Stülke, 2005; Thevenot et al., 1995). For instance, in S. thermophilus, lactose is taken up by the Lactose $/ \mathrm{H}^{+}$ symporter LacS. The rate of Lactose uptake is inhibited three-fold when IIA-like domain of LacS is phosphorylated by $\mathrm{HPr}(\mathrm{His}-\mathrm{P})$ at a regulatory histidine residue. The rate of reversible phosphorylation of LacS was found to be the same regardless whether $\mathrm{HPr}(\mathrm{His}-\mathrm{P})$ or $\mathrm{HPr}(\mathrm{Ser}-$ P)(His-P) is used as phosphate donor (Cochu et al., 2005). However, in B. subtilis, role of $\mathrm{HPr}($ Ser-P)(His-P) in participating in PTS transport functions appears less likely. The HPr S46D variant from B. subtilis, which mimics the negative charge of phosphorylation at serine 
46 (S46D), shows a markedly reduced rates of PTS sugar uptake when expressed in a heterologous system (S. aureus) (Reizer et al., 1989). Moreover, the doubly phosphorylated form was only detected in the presence of non-PTS sugars in this work except for maltose.

\subsection{Activity of HPrK/P determines CCR in B. subtilis}

It was suggested that EI can compete with the kinase activity of HPrK/P leading to a reduced level of $\mathrm{HPr}(\mathrm{Ser}-\mathrm{P})$. This was explained by the common interacting surface employed by HPr to bind HPrK/P and EI (Fieulaine et al., 2002; Reizer et al., 1989). The presence of weakly repressing carbon sources generated low levels of intracellular $\operatorname{HPr}(\mathrm{Ser}-\mathrm{P})$ in the cell and considerable amount of $\mathrm{HPr}(\mathrm{His}-\mathrm{P})$. This suggested that the PEP dependent phosphorylation of HPr limits the HPr(Ser-P) amounts in the cell in these carbon sources. An EI mutant however, did not show any increased CCR. In the absence of EI, neither HPr is phosphorylated at His-15 nor is it occupied by EI, thus avoiding any competition for HPrK/P. This leads to the conclusion that the phosphorylation at His-15 of HPr does not negatively regulate the phosphorylation at Ser-46 achieved by HPrK/P. Thus, the $\mathrm{HPr}(\mathrm{Ser}-\mathrm{P})$ levels in the cell are exclusively determined by HPrK/P kinase activity. Moreover, results from this work and previously published reports support this idea. In the presence of HPrK/P variants, which lost their phosphorylase activity, and acts a constitutive kinase, a permanent state of repression was observed (Monedero et al., 2001; Singh et al., 2008). The constitutive kinase HPrK/P generates high amounts of intracellular $\mathrm{HPr}(\mathrm{Ser}-\mathrm{P})$ even in the absence of a repressing carbon source (Monedero et al., 2001; Singh et al., 2008).

Most of the strongly repressing carbon sources are catabolized via glycolysis (see Fig. 2). It was also demonstrated that glucose drastically lost its repressing potential on the iol operon, when the flux of glucose utilization is directed towards PPP, rather than glycolysis (Nihashi and Fujita, 1984). Interestingly, the uptake of maltose, which is also a glycolytic sugar, did not lead to very strong repression. Catabolite repression studies using maltose as a potential sugar, considered it a weakly metabolized carbon source (Nihashi and Fujita, 1984). This is also supported by observations in this work where large amounts of $\operatorname{HPr}(\mathrm{His}-\mathrm{P})$ and less $\operatorname{HPr}(\operatorname{Ser}-\mathrm{P})$ were observed in the presence of maltose. Maltose is a PTS sugar and probably a low flux of PTS towards the maltose specific permease, limits uptake of maltose. This may lead to lower levels of $\operatorname{HPr}(\operatorname{Ser}-\mathrm{P})$ because only low amounts of glycolytic intermediates including FBP are formed. 
In vitro, $\mathrm{HPrK} / \mathrm{P}$ exhibits kinase activity above $1 \mathrm{mM}$ FBP and maximum activity at $5 \mathrm{mM}$ FBP concentrations (Jault et al., 2000). Here, we showed that $1.8 \mathrm{mM} \mathrm{FBP}$ is present intracellulary in the absence of a repressing sugar. In the presence of various other sugars, FBP concentrations in the range of 4.3-14.1 $\mathrm{mM}$ were detected. Therefore all sugars generated FBP levels, which are theoretically sufficient for a high kinase activity. The question that still holds is how the kinase activity of HPrK/P is modulated by the different carbon sources. Various possibilities could explain the differential kinase activities of HPrK/P. For instance, there might be an alternative allosteric activator of kinase activity. The role of FBP as a regulator of CCR has been demonstrated very coherently in B. subtilis. Any mutation that prevents the formation of FBP in vivo results in a complete relief of CCR (Nihashi and Fujita, 1984). Moreover, in vitro studies using several other intermediates of glycolysis for e.g G6P, fructose-6-P, 2-P-glycerate and of PPP such as gluconate-6-P, excluded the role of these metabolite in stimulating the kinase activity of HPrK/P (Reizer et al., 1998). The role of G6P in CCR has been implicated previously. Besides FBP, G6P has been suggested to modulate the CcpA/HPr(Ser-P) complex (Siedel et al., 2005; Schumacher et al., 2007). In this respect the intracellular amounts of G6P were estimated in wt cells in the presence of various carbon sources. Glucose generated highest levels of intracellular G6P, however there was no strict correlation between the strength of repression and G6P levels, in the presence of other carbon sources (see table 17 in supplementary material).

As an activator of HPrK/P kinase activity, FBP seems to play the most important role in B. subtilis. It is also possible that the effect of FBP on HPrK/P is similar under all conditions, but unknown signal antagonises the effect of FBP. As shown for the L. casei HPrK/P, the phosphate from the ATP binds at the P-loop and the base moiety of ATP interacts with Trp-237 of HPrK/P. This interaction stabilizes the kinase conformation (Chaptal et al., 2007) Inorganic Pi, the substrate for the HPrK/P mediated dephosphorylation, occupies the position of the $\beta$ - and $\gamma$-phosphate of ATP in the P-loop of L. casei HPrK/P (Fieulaine et al., 2001). This explains why $\mathrm{P}_{\mathrm{i}}$ is an inhibitor os the kinase reaction. In contrast to most bifunctional enzymes, such as aspartokinaseI/ homoserine dehydrogenase I (Jullien et al., 1988) or 6-phosphofructo-2-kinase/fructose-2,6-bisphosphatase (Yang et al., 2001), which possess two distinct active sites, the activities of HPrK/P are catalysed by the same site. Thus, the two conformations are probably in an $\mathrm{ATP} / \mathrm{P}_{\mathrm{i}}$ regulated equilibrium. This concept of equilibrium shift activation of antagonistic activities has been also observed for E. coli CheY (Dyer and Dahlquist, 2006). In vitro, the FBP dependent stimulation of the ATP dependent kinase activity is more obvious at ATP concentrations lower than $0.4 \mathrm{mM}$. As mentioned 
before, the maximum kinase activity can be achieved already at $5 \mathrm{mM}$ FBP concentrations. Interestingly, the phosphorylation achieved by HPrK/P in the presence of $0.5 \mathrm{mM}$ ATP and 5 $\mathrm{mM}$ FBP can be totally inhibited by the presence of $0.5 \mathrm{mM} \mathrm{P} \mathrm{P}_{\mathrm{i}}$ (Lavergne et al., 2002). Thus, it is conceivable that relative changes in the intracellular ratio of ATP and $\mathrm{P}_{\mathrm{i}}$ may affect the kinase conformation. Similarly, for S. bovis, an inverse relationship between the HPr(Ser-P) formation and the Pi concentration was observed (Asanuma and Hino, 2003).

An alternate mechanism, which could explain the low HPrK/P kinase activity in the presence of weakly repressing carbon sources, might be the binding of HPrK/P by an unknown inhibitor in a non competitive manner. In support of this, a relief from CCR was observed, when HPrK/P was expressed ectopically. Therefore, one possibility is that a protein encoded downstream of $h p r K$ in the same operon, opposes HPrK/P activity.

It was suggested that the $\mathrm{N}$-terminal domain of $\mathrm{HPrK} / \mathrm{P}$, whose function is unknown can interact with phosphorylated metabolites. However, the truncated L. casie HPrK/P missing the first 127 amino acids exhibited both enzymatic activities and responded to all known effectors in the same way as the wild type enzyme (Fieulaine et al., 2001). Thus, FBP, ATP and Pi binding sites reside in the C-terminal domain. The N-terminal domain, which protrudes from the core hexamer exhibits similarity to UDP- $N$-acetylmuramoyl- ${ }_{\mathrm{L}}-$ alanyl- ${ }_{\mathrm{D}^{-}}$ glutamate: meso-diaminopimelate ligase (MurE) of E. coli (Poncet et al., 2004). MurE is an amide bond ligase involved in cell wall synthesis (Smith, 2006). This resemblance between the N-terminal domain of HPrK/P and the UDP interacting site of MurE, suggested that the $\mathrm{N}$-terminal part of HPrK/P may interact with phosphorylated metabolites. Moreover, it has been demonstrated in vitro that a number of metabolic intermediates can inhibit the HPrK/P kinase activity including acetyl phosphate and glyceraldehyde phosphate (Ramström et al., 2003). Acetyl phosphate has been postulated to be an indicator of glucose starvation. Synthesis of acetyl phosphate was found to be necessary for glucose starved cells of $E$. coli to survive glucose starvation (Nystrom, 1994). In this respect, it would be very interesting for the future studies to test the effect of an N-terminally truncated version of HPrK/P on CCR and on the phosphorylation pattern of HPr in vivo. 


\section{Summary and Conclusions}

CCR is one of the most thoroughly investigated signal transduction system in bacteria. It allows the bacteria to adapt to changes in the availability and supply of different carbon sources. In general, CCR is defined as the selective utilization of a preferred carbon source which represses the functions for the utilization of secondary carbon sources. At the molecular level, CCR is achieved by the global transcriptional regulator CcpA, in B. subtilis. CcpA forms a repressor complex with serine phosphorylated HPr and Crh proteins in the presence of a preferred carbon source like glucose. This repressor complex binds to the operator sites on the DNA called cre sites and thereby repressing a number of catabolic genes and operons involved in utilization of secondary carbon sources. The regulatory phosphorylation of HPr and Crh is achieved by a bifunctional enzyme HPrK/P.

In this work, the repressing potential of various carbon sources besides glucose was analysed. A number of carbon sources could exert CcpA-mediated catabolite repression of the reporter system. Moreover, the substrates formed a hierarchy in their ability to exert repression. The different levels of repression by various carbon sources indicated the formation of the CcpA/co-repressor complex to different extents. CcpA and HPr levels were found to be similar in the cell, irrespective of the nature of the carbon source used. Thus, the phosphorylation pattern of the co-repressor was analysed. As a prerequisite for this experiment, it could be established that HPr and not Crh is the relevant co-repressor of CcpA. Thus, the focus was on analysing the level of $\operatorname{HPr}(\operatorname{Ser}-\mathrm{P})$ in the cell. The presence of strong repressing carbon sources generated high intracellular $\mathrm{HPr}(\mathrm{Ser}-\mathrm{P})$ as compared to the poor repressing carbon sources. Thus, it could be well established that the different repressing potential of various carbon sources is derived from the ability to generate different intracellular levels of $\mathrm{HPr}(\operatorname{Ser}-\mathrm{P})$.

In the presence of poor repressing carbon sources, besides low intracellular $\mathrm{HPr}(\mathrm{Ser}-$ P), considerable amounts of histidine phosphorylated HPr were also present. HPr is also a part of the sugar PTS. The PTS is involved in the concomitant uptake and phosphorylation of various carbon sources. As a part of PTS, HPr is phosphorylated at its His-15 residue and receives phosphate from PEP via EI. Phosphorylation at histidine residue might decrease the available $\mathrm{HPr}$ as a substrate for HPrK/P. Therefore, the possibility that the presence of $\mathrm{HPr}($ His-P) negatively regulates the phosphorylation of HPr by HPrK/P was addressed. In an EI mutant, $\operatorname{HPr}($ His-P) is not formed. However, the repression potential of the non-PTS carbon sources remained unchanged in the EI mutant. This clearly established that the 
phosphorylation of HPr at Ser-46 is exclusively determined by HPrK/P activity and not by the PTS. Thus, in the presence of weaker repressing carbon sources, low $\mathrm{HPr}(\mathrm{Ser}-\mathrm{P})$ levels are generated owing to the low kinase activity of HPrK/P. This hypothesis was confirmed by using an HPrK/P variant, which lost its phosphorylase activity. Such a variant exhibited a low but constitutive kinase activity and allowed repression even in the absence of a repressing carbon source.

Modulation of the HPrK/P activity is suggested to be achieved by an allosteric regulation by metabolites like FBP and $\mathrm{P}_{\mathrm{i}}$. Therefore, we determined the FBP concentration in vivo. It turned out that on most sugars the intracellular FBP level is high enough to achieve theoretically a complete activation of the HPrK/P kinase activity. Therefore, further factors should exist that regulate HPrK/P activity in vivo.

To probe into the possibility that $\operatorname{HPr}(\operatorname{Ser}-\mathrm{P})$ levels can be affected by an enzyme other than HPrK/P, the role of PrpC was analysed. PrpC, a Ser/Thr phosphatase, has shown to dephosphorylate $\operatorname{HPr}(\operatorname{Ser}-\mathrm{P})$ in $M$. pneumoniae. B. subtilis PrpC could dephosphorylate $\mathrm{HPr}(\mathrm{Ser}-\mathrm{P})$ in vitro, but its absence or presence had no effect on CCR in vivo.

Taken together, this work coherently demonstrates the central role of HPrK/P in CCR in B. subtilis. Moreover, the hierarchy of repression exerted by various carbon sources could be well explained with the levels of $\operatorname{HPr}(\operatorname{Ser}-\mathrm{P})$ generated in the cell. This work further highlights the fundamental differences in the mechanism of catabolite repression in the two model organisms E. coli and B. subtilis. PTS transport activity is the decisive factor of both global and operon-specific CCR in E. coli. In the absence of $\mathrm{HPr}(\mathrm{His}-\mathrm{P})$ in E. coli, EIIA ${ }^{\text {glu }}$ is rendered unphosphorylated and exerts strong catabolite repression. In contrast, the global CCR mechanism in B. subtilis is not directly affected by the PTS activity. 


\section{References}

Adler,E., Donella-Deana,A., Arigoni,F., Pinna,L.A., and Stragler,P. (1997) Structural relationship between a bacterial developmental protein and eukaryotic PP2C protein phosphatases. Mol Microbiol 23: 57-62.

Alex,L.A., and Simon,M.I. (1994) Protein histidine kinases and signal transduction in prokaryotes and eukaryotes. Trends Genet 10: 133-138.

Allen,G.S., Steinhauer,K., Hillen,W., Stülke,J., and Brennan,R.G. (2003) Crystal structure of HPr kinase/phosphatase from Mycoplasma pneumoniae. J Mol Biol 326: 1203-1217.

Arnaud,M., Debarbouille,M., Rapoport,G., Saier,M.H., Jr., and Reizer,J. (1996) In vitro reconstitution of transcriptional antitermination by the SacT and SacY proteins of Bacillus subtilis. J Biol Chem 271: 18966-18972.

Asanuma,N., and Hino,T. (2003) Molecular characterization of HPr and related enzymes, and regulation of HPr phosphorylation in the ruminal bacterium Streptococcus bovis. Arch Microbiol 179: 205-213.

Aung-Hilbrich,L.M., Seidel,G., Wagner,A., and Hillen,W. (2002) Quantification of the influence of HPrSer46P on CcpA-cre interaction. J Mol Biol 319: 77-85.

Aymerich,S., and Steinmetz,M. (1992) Specificity determinants and structural features in the RNA target of the bacterial antiterminator proteins of the BglG/SacY family. Proc Natl Acad Sci U S A 89: 10410-4.

Bachem,S., and Stülke,J. (1998) Regulation of the Bacillus subtilis GlcT antiterminator protein by components of the phosphotransferase system. J Bacteriol 180: 5319-5326.

Beijer,L., and Rutberg,L. (1992) Utilisation of glycerol and glycerol 3-phosphate is differently affected by the phosphotransferase system in Bacillus subtilis. FEMS Microbiol Lett 79: 217-220.

Bettenbrock,K., Sauter,T., Jahreis,K., Kremling,A., Lengeler,J.W., and Gilles,E.D. (2007) Correlation between growth rates, EIIA ${ }^{\mathrm{Crr}}$ phosphorylation, and intracellular cyclic AMP levels in Escherichia coli K-12. J Bacteriol 189: 6891-900.

Blencke,H.M., Homuth,G., Ludwig,H., Mader,U., Hecker,M., and Stülke,J. (2003) Transcriptional profiling of gene expression in response to glucose in Bacillus subtilis: regulation of the central metabolic pathways. Metab Eng 5: 133-149.

Belitsky,B.R., Kim,H.J., and Sonenshein,A.L. (2004) CcpA-dependent regulation of Bacillus subtilis glutamate dehydrogenase gene expression. J Bacteriol 186: 3392-3398.

Belitsky,B.R., and Sonenshein,A.L. (1998) Role and regulation of Bacillus subtilis glutamate dehydrogenase genes. J Bacteriol 180: 6298-6305. 
Boël,G., Mijakovic,I., Maze,A., Poncet,S., Taha,M.K., Larribe,M. et al. (2003) Transcription regulators potentially controlled by HPr kinase/phosphorylase in Gram-negative bacteria. $J$ Mol Microbiol Biotechnol 5: 206-215.

Breeuwer,P., Drocourt,J., Rombouts,F.M., and Abee,T. (1996) A Novel Method for Continuous Determination of the Intracellular $\mathrm{pH}$ in Bacteria with the Internally Conjugated Fluorescent Probe 5 (and 6-)-Carboxyfluorescein Succinimidyl Ester. Appl Environ Microbiol 62: $178-183$.

Cai,S.J., and Inouye,M. (2002) EnvZ-OmpR interaction and osmoregulation in Escherichia coli. J Biol Chem 277: 24155-24161.

Casabon,I., Couture,M., Vaillancourt,K., and Vadeboncoeur,C. (2006) Synthesis of HPr(SerP)(His-P) by enzyme I of the phosphoenolpyruvate: sugar phosphotransferase system of Streptococcus salivarius. Biochemistry 45: 6692-6702.

Chalumeau,H., Delobbe,A., and Gay,P. (1978) Biochemical and genetic study of D-glucitol transport and catabolism in Bacillus subtilis. J Bacteriol 134: 920-928.

Chaptal,V., Vincent,F., Gueguen-Chaignon,V., Monedero,V., Poncet,S., Deutscher,J. et al. (2007) Structural analysis of the bacterial HPr kinase/phosphorylase V267F mutant gives insights into the allosteric regulation mechanism of this bifunctional enzyme. $J$ Biol Chem 282: 34952-34957.

Cochu,A., Roy,D., Vaillancourt,K., Lemay,J.D., Casabon,I., Frenette,M. et al. (2005) The doubly phosphorylated form of $\operatorname{HPr}, \operatorname{HPr}(\operatorname{Ser} \sim \mathrm{P})(\mathrm{His}-\mathrm{P})$, is abundant in exponentially growing cells of Streptococcus thermophilus and phosphorylates the lactose transporter LacS as efficiently as HPr(His P). Appl Environ Microbiol 71: 1364-1372.

Commichau,F.M., Gunka,K., Landmann,J.J., and Stülke,J. (2008) Glutamate metabolism in Bacillus subtilis: gene expression and enzyme activities evolved to avoid futile cycles and to allow rapid responses to perturbations of the system. J Bacteriol 190: 3557-3564.

Commichau,F.M., Wacker,I., Schleider,J., Blencke,H.M., Reif,I., Tripal,P., and Stülke,J. (2007) Characterization of Bacillus subtilis mutants with carbon source-independent glutamate biosynthesis. J Mol Microbiol Biotechnol 12: 106-113.

Cozzone,A.J. (1988) Protein phosphorylation in prokaryotes. Annu Rev Microbiol 42: 97-125.

Cozzone,A.J. (1993) ATP-dependent protein kinases in bacteria. J Cell Biochem 51: 7-13.

Crutz,A.M., Steinmetz,M., Aymerich,S., Richter,R., and Le Coq,D. (1990) Induction of levansucrase in Bacillus subtilis: an antitermination mechanism negatively controlled by the phosphotransferase system. J Bacteriol 172: 1043-50.

Dahl,M.K., Degenkolb,J., and Hillen,W. (1994) Transcription of the $x y l$ operon is controlled in Bacillus subtilis by tandem overlapping operators spaced by four base-pairs. $J$ Mol Biol 243: 413-424.

Dahl,M.K., Schmiedel,D., and Hillen,W. (1995) Glucose and glucose-6-phosphate interaction with Xyl repressor proteins from Bacillus spp. may contribute to regulation of xylose utilization. J Bacteriol 177: 5467-5472. 
Darbon,E., Servant,P., Poncet,S., and Deutscher,J. (2002) Antitermination by GlpP, catabolite repression via CcpA and inducer exclusion triggered by P-GlpK dephosphorylation control Bacillus subtilis glpFK expression. Mol Microbiol 43: 1039-1052.

Das,A.K., Helps,N.R., Cohen,P.T., and Barford,D. (1996) Crystal structure of the protein serine/threonine phosphatase $2 \mathrm{C}$ at 2.0 A resolution. EMBO J 15: 6798-6809.

Deutscher,J. (2008) The mechanisms of carbon catabolite repression in bacteria. Curr Opin Microbiol 11: 87-93.

Deutscher,J., Francke,C., and Postma,P.W. (2006) How phosphotransferase system-related protein phosphorylation regulates carbohydrate metabolism in bacteria. Microbiol Mol Biol Rev 70: 939-1031.

Deutscher,J., Galinier,A., and Martin-Verstraete,I. (2002) Carbohydate uptake and metabolism. In: A. L Sonenshein, J. A. Hoch, and R. Losick (ed.), Bacillus subtilis and its closest relatives. ASM press, Washington, D.C. p. 129-150.

Deutscher,J., Kessler,U., Alpert,C.A., and Hengstenberg W. (1984) Bacterial phosphoenolpyruvate-dependent phosphotransferase system: P-ser-HPr and its possible regulatory function Biochemistry 23:4455-4460.

Deutscher,J., Reizer,J., Fischer,C., Galinier,A., Saier,M.H., Jr., and Steinmetz,M. (1994) Loss of protein kinase-catalyzed phosphorylation of HPr, a phosphocarrier protein of the phosphotransferase system, by mutation of the $p t s H$ gene confers catabolite repression resistance to several catabolic genes of Bacillus subtilis. J Bacteriol 176: 3336-3344.

Deutscher,J., and Saier,M.H., Jr. (2005) Ser/Thr/Tyr protein phosphorylation in bacteria - for long time neglected, now well established. J Mol Microbiol Biotechnol 9: 125-131.

Deutscher,J., and Saier,M.H., Jr. (1983) ATP-dependent protein kinase-catalyzed phosphorylation of a seryl residue in HPr, a phosphate carrier protein of the phosphotransferase system in Streptococcus pyogenes. Proc Natl Acad Sci U S A 80: 67906794.

Dimitrova,M.N., Peterkofsky,A., and Ginsburg,A. (2003) Opposing effects of phosphoenolpyruvate and pyruvate with $\mathrm{Mg}(2+)$ on the conformational stability and dimerization of phosphotransferase enzyme I from Escherichia coli. Protein Sci 12: 20472056.

Doan,T., and Aymerich,S. (2003) Regulation of the central glycolytic genes in Bacillus subtilis: binding of the repressor CggR to its single DNA target sequence is modulated by fructose-1,6-bisphosphate. Mol Microbiol 47: 1709-1721.

Dossonnet,V., Monedero,V., Zagorec,M., Galinier,A., Perez-Martinez,G., and Deutscher,J. (2000) Phosphorylation of HPr by the bifunctional HPr Kinase/P-ser-HPr phosphatase from Lactobacillus casei controls catabolite repression and inducer exclusion but not inducer expulsion. J Bacteriol 182: 2582-2590.

Dyer,C.M., and Dahlquist,F.W. (2006) Switched or not?: the structure of unphosphorylated CheY bound to the N terminus of FliM. J Bacteriol 188: 7354-7363. 
Duncan,L., Alper,S., Arigoni,F., Losick,R., and Stragier,P. (1995) Activation of cell-specific transcription by a serine phosphatase at the site of asymmetric division. Science 270: 641-644.

Errington,J. (1993) Bacillus subtilis sporulation: regulation of gene expression and control of morphogenesis. Microbiol Rev 57: 1-33.

Fabret,C., Feher,V.A., and Hoch,J.A. (1999) Two-component signal transduction in Bacillus subtilis: how one organism sees its world. J Bacteriol 181: 1975-1983.

Faires,N., Tobisch,S., Bachem,S., Martin-Verstraete,I., Hecker,M., and Stülke,J. (1999) The catabolite control protein CcpA controls ammonium assimilation in Bacillus subtilis. $\mathrm{J}$ Mol Microbiol Biotechnol 1: 141-148.

Feucht,A., and Lewis,P.J. (2001) Improved plasmid vectors for the production of multiple fluorescent protein fusions in Bacillus subtilis. Gene 264: 289-297.

Fieulaine,S., Morera,S., Poncet,S., Mijakovic,I., Galinier,A., Janin,J. et al. (2002) X-ray structure of a bifunctional protein kinase in complex with its protein substrate HPr. Proc Natl Acad Sci U S A 99: 13437-13441.

Fieulaine,S., Morera,S., Poncet,S., Monedero,V., Gueguen-Chaignon,V., Galinier,A. et al. (2001) X-ray structure of HPr kinase: a bacterial protein kinase with a P-loop nucleotidebinding domain. EMBO J 20: 3917-3927.

Fujita,Y., Miwa,Y., Tojo,S., and Hirooka,K. (2007) Carbon catabolite control and metabolic networks mediated by the CcpA protein in Bacillus subtilis. In Global regulatory networks in Bacillus subtilis. Fujita,Y. (ed). Trivandrum, India: Transworld Research Network, pp. 91110.

Gaidenko,T.A., Kim,T.J., and Price,C.W. (2002) The PrpC serine-threonine phosphatase and PrkC kinase have opposing physiological roles in stationary-phase Bacillus subtilis cells. $J$ Bacteriol 184: 6109-6114.

Galinier,A., Deutscher,J., and Martin-Verstraete,I. (1999) Phosphorylation of either Crh or HPr mediates binding of CcpA to the Bacillus subtilis xyn cre and catabolite repression of the xyn operon. J Mol Biol 286: 307-314.

Galinier,A., Haiech,J., Kilhoffer,M.C., Jaquinod,M., Stülke,J., Deutscher,J., and MartinVerstraete,I. (1997) The Bacillus subtilis crh gene encodes a HPr-like protein involved in carbon catabolite repression. Proc Natl Acad Sci U S A 94: 8439-8444.

Galinier,A., Kravanja,M., Engelmann,R., Hengstenberg,W., Kilhoffer,M.C., Deutscher,J., and Haiech,J. (1998) New protein kinase and protein phosphatase families mediate signal transduction in bacterial catabolite repression. Proc Natl Acad Sci U S A 95: 1823-1828.

Garnak,M., and Reeves,H.C. (1979) Phosphorylation of Isocitrate dehydrogenase of Escherichia coli. Science 203: 1111-1112.

Garrett,D.S., Seok,Y.J., Peterkofsky,A., Gronenborn,A.M., and Clore,G.M. (1999) Solution structure of the 40,000 Mr phosphoryl transfer complex between the N-terminal domain of enzyme I and HPr. Nat Struct Biol 6: 166-173. 
Gauthier,M., Brochu,D., Eltis,L.D., Thomas,S., and Vadeboncoeur,C. (1997) Replacement of isoleucine- 47 by threonine in the HPr protein of Streptococcus salivarius abrogates the preferential metabolism of glucose and fructose over lactose and melibiose but does not prevent the phosphorylation of HPr on serine-46. Mol Microbiol 25: 695-705.

Görke,B., and Deutscher,J. (2007) The regulatory functions of histidyl-phosphorylated HPr in bacilli. In: Fujita,Y. (ed), Global regulatory networks in Bacillus subtilis. Trivandrum, India: Transworld Research Network, pp. 1-37.

Görke,B., Fraysse,L., and Galinier,A. (2004) Drastic differences in Crh and HPr synthesis levels reflect their different impacts on catabolite repression in Bacillus subtilis. J Bacteriol 186: $2992-5$.

Görke,B., and Rak,B. (1999) Catabolite control of Escherichia coli regulatory protein BglG activity by antagonistically acting phosphorylations. Embo J 18: 3370-3379.

Görke,B., and Stülke,J. (2008) Carbon catabolite repression in bacteria: many ways to make the most out of nutrients. Nat Rev Microbiol 6: 613-624.

Gosset,G., Zhang,Z., Nayyar,S., Cuevas,W.A., and Saier,M.H., Jr. (2004) Transcriptome analysis of Crp-dependent catabolite control of gene expression in Escherichia coli. $J$ Bacteriol 186: 3516-3524.

Gough,J.A., and Murray,N.E. (1983) Sequence diversity among related genes for recognition of specific targets in DNA molecules. J Mol Biol 166: 1-19.

Guan,K.L., and Dixon,J.E. (1990) Protein tyrosine phosphatase activity of an essential virulence determinant in Yersinia. Science 249: 553-556.

Guerout-Fleury,A.M., Shazand,K., Frandsen,N., and Stragier,P. (1995) Antibiotic-resistance cassettes for Bacillus subtilis. Gene 167: 335-336.

Halbedel, S. (2006) Regulation of HPr phosphorylation in Mycoplasma pneumoniae. PhD thesis, University of Göttingen.

Halbedel,S., Busse,J., Schmidl,S.R., and Stülke,J. (2006) Regulatory protein phosphorylation in Mycoplasma pneumoniae. A PP2C-type phosphatase serves to dephosphorylate HPr(SerP). J Biol Chem 281: 26253-26259.

Halbedel,S., and Stülke,J. (2005) Dual phosphorylation of Mycoplasma pneumoniae HPr by Enzyme I and HPr kinase suggests an extended phosphoryl group susceptibility of HPr. FEMS Microbiol Lett 247: 193-198.

Hanson,K.G., Steinhauer,K., Reizer,J., Hillen,W., and Stülke,J. (2002) HPr kinase/phosphatase of Bacillus subtilis: expression of the gene and effects of mutations on enzyme activity, growth and carbon catabolite repression. Microbiology 148: 1805-1811.

Hellingwerf,K.J., Crielaard,W.C., Mattos-Teixeira, M.J., Hoff, D.W., Kort, R., Verhamme, T., and Rossa-Avignone, C (1998) Current topics in signal transduction in bacteria. Antonie van Leeuwenhoek 74: 211-227. 
Henkin,T.M., Grundy,F.J., Nicholson,W.L., and Chambliss,G.H. (1991) Catabolite repression of alpha-amylase gene expression in Bacillus subtilis involves a trans-acting gene product homologous to the Escherichia coli lacl and galR repressors. Mol Microbiol 5: 575-584.

Herzberg,C., Weidinger,L.A., Dorrbecker,B., Hubner,S., Stülke,J., and Commichau,F.M. (2007) SPINE: a method for the rapid detection and analysis of protein-protein interactions in vivo. Proteomics 7: 4032-4035.

Himmelreich,R., Hilbert,H., Plagens,H., Pirkl,E., Li,B.C., and Herrmann,R. (1996) Complete sequence analysis of the genome of the bacterium Mycoplasma pneumoniae. Nucleic Acids Res 24: 4420-4449.

Hogema,B.M., Arents,J.C., Bader,R., Eijkemans,K., Yoshida,H., Takahashi,H. et al. (1998) Inducer exclusion in Escherichia coli by non-PTS substrates: the role of the PEP to pyruvate ratio in determining the phosphorylation state of enzyme IIA ${ }^{\text {Glc }}$. Mol Microbiol 30: 487-498.

Hogema,B.M., Arents,J.C., Inada,T., Aiba,H., van Dam,K., and Postma,P.W. (1997) Catabolite repression by glucose 6-phosphate, gluconate and lactose in Escherichia coli. Mol Microbiol 24: 857-867.

Huffine,M.E., and Scholtz,J.M. (1996) Energetic implications for protein phosphorylation. Conformational stability of HPr variants that mimic phosphorylated forms. J Biol Chem 271: 28898-28902.

Hughes, K.T., and Mathee, K. (1998) The anti-sigma factors. Annu Rev Microbiol 52:231286.

Hunter,T. (2000) Signaling--2000 and beyond. Cell 100: 113-127.

Igarashi,T., Setlow,B., Paidhungat,M., and Setlow,P. (2004) Effects of a gerF (lgt) mutation on the germination of spores of Bacillus subtilis. J Bacteriol 186: 2984-2991.

Inacio,J.M., Costa,C., and de Sa-Nogueira,I. (2003) Distinct molecular mechanisms involved in carbon catabolite repression of the arabinose regulon in Bacillus subtilis. Microbiology 149: $2345-2355$.

Inacio,J.M., and de Sa-Nogueira,I. (2007) trans-Acting factors and cis elements involved in glucose repression of arabinan degradation in Bacillus subtilis. J Bacteriol 189: 8371-8376.

Jault,J.M., Fieulaine,S., Nessler,S., Gonzalo,P., Di Pietro,A., Deutscher,J., and Galinier,A. (2000) The HPr kinase from Bacillus subtilis is a homo-oligomeric enzyme which exhibits strong positive cooperativity for nucleotide and fructose 1,6-bisphosphate binding. J Biol Chem 275: 1773-1780.

Johnson,L.N., and Barford,D. (1993) The effects of phosphorylation on the structure and function of proteins. Annu Rev Biophys Biomol Struct 22: 199-232.

Jones,B.E., Dossonnet,V., Küster,E., Hillen,W., Deutscher,J., and Klevit,R.E. (1997) Binding of the catabolite repressor protein CcpA to its DNA target is regulated by phosphorylation of its corepressor HPr. J Biol Chem 272: 26530-26535. 
Jordan,S., Junker,A., Helmann,J.D., and Mascher,T. (2006) Regulation of LiaRS-dependent gene expression in bacillus subtilis: identification of inhibitor proteins, regulator binding sites, and target genes of a conserved cell envelope stress-sensing two-component system. $J$ Bacteriol 188: 5153-5166.

Joseph,P., Fantino,J.R., Herbaud,M.L., and Denizot,F. (2001) Rapid orientated cloning in a shuttle vector allowing modulated gene expression in Bacillus subtilis. FEMS Microbiol Lett 205: 91-97.

Jullien,M., Baudet,S., Rodier,F., and Le,B.G. (1988) Allosteric transition of aspartokinase Ihomoserine dehydrogenase I studied by time-resolved fluorescence. Biochimie 70: 18071814.

Kaltwasser,M., Wiegert,T., and Schumann,W. (2002) Construction and application of epitope- and green fluorescent protein-tagging integration vectors for Bacillus subtilis. Appl Environ Microbiol 68: 2624-2628.

Kennelly,P.J., and Potts,M. (1996) Fancy meeting you here! A fresh look at "prokaryotic" protein phosphorylation. J Bacteriol 178: 4759-4764.

Kim,J.H., and Chambliss,G.H. (1997) Contacts between Bacillus subtilis catabolite regulatory protein CcpA and amyO target site. Nucleic Acids Res 25: 3490-3496.

Kraus,A., and Hillen,W. (1997) Analysis of CcpA mutations defective in carbon catabolite repression in Bacillus megaterium. FEMS Microbiol Lett 153: 221-226.

Kraus,A., Hueck,C., Gärtner,D., and Hillen,W. (1994) Catabolite repression of the Bacillus subtilis xyl operon involves a cis element functional in the context of an unrelated sequence, and glucose exerts additional $x y l R$-dependent repression. J Bacteriol 176: 1738-1745.

Kraus,A., Küster,E., Wagner,A., Hoffmann,K., and Hillen,W. (1998) Identification of a corepressor binding site in catabolite control protein CcpA. Mol Microbiol 30: 955-963.

Kravanja,M., Engelmann,R., Dossonnet,V., Bluggel,M., Meyer,H.E., Frank,R. et al. (1999) The hprK gene of Enterococcus faecalis encodes a novel bifunctional enzyme: the HPr kinase/phosphatase. Mol Microbiol 31: 59-66.

Krispin,O., and Allmansberger,R. (1998) The Bacillus subtilis AraE protein displays a broad substrate specificity for several different sugars. J Bacteriol 180: 3250-3252.

Krüger,S., Gertz,S., and Hecker,M. (1996) Transcriptional analysis of bglPH expression in Bacillus subtilis: evidence for two distinct pathways mediating carbon catabolite repression. $J$ Bacteriol 178: 2637-44.

Krüger,S., and Hecker,M. (1995) Regulation of the putative bglPH operon for aryl-betaglucoside utilization in Bacillus subtilis. J Bacteriol 177: 5590-7.

Kunst,F., Ogasawara,N., Moszer,I., Albertini,A.M., Alloni,G., Azevedo,V. et al. (1997) The complete genome sequence of the gram-positive bacterium Bacillus subtilis. Nature 390: 249256.

Kunst,F., and Rapoport,G. (1995) Salt stress is an environmental signal affecting degradative enzyme synthesis in Bacillus subtilis. J Bacteriol 177: 2403-2407. 
Küster,E., Luesink,E.J., de Vos,W.M., and Hillen,W. (1996) Immunological crossreactivity to the catabolite control protein CcpA from Bacillus megaterium is found in many gram-positive bacteria. FEMS Microbiol Lett 139: 109-115.

Küster-Schöck,E., Wagner,A., Volker,U., and Hillen,W. (1999) Mutations in catabolite control protein CcpA showing glucose-independent regulation in Bacillus megaterium. $J$ Bacteriol 181: 7634-7638.

LaPorte,D.C. (1993) The isocitrate dehydrogenase phosphorylation cycle: regulation and enzymology. J Cell Biochem 51: 14-18.

LaPorte,D.C., and Koshland,D.E., Jr. (1982) A protein with kinase and phosphatase activities involved in regulation of tricarboxylic acid cycle. Nature 300: 458-460.

Lavergne,J.P., Jault,J.M., and Galinier,A. (2002) Insights into the functioning of Bacillus subtilis HPr kinase/phosphatase: affinity for its protein substrates and role of cations and phosphate. Biochemistry 41: 6218-6225.

Leboeuf,C., Leblanc,L., Auffray,Y., and Hartke,A. (2000) Characterization of the ccpA gene of Enterococcus faecalis: identification of starvation-inducible proteins regulated by ccpA. $J$ Bacteriol 182: 5799-5806.

Lereclus,D., and Arantes,O. (1992) spbA locus ensures the segregational stability of pTH1030, a novel type of gram-positive replicon. Mol Microbiol 6: 35-46.

Leskelä,S., Wahlstrom,E., Kontinen,V.P., and Sarvas,M. (1999a) Lipid modification of prelipoproteins is dispensable for growth but essential for efficient protein secretion in Bacillus subtilis: characterization of the Lgt gene. Mol Microbiol 31: 1075-1085.

Lévine A, Vannier F, Absalon C, Kuhn L, Jackson P, Scrivener E, Labas V, Vinh J, Courtney P, Garin J, Séror SJ: Analysis of the dynamic Bacillus subtilis Ser/ Thr/ Tyr phosphoproteome implicated in a wide variety of cellular processes. Proteomics 2006, 6:2157-2173.

Lewis,P.J., and Marston,A.L. (1999) GFP vectors for controlled expression and dual labelling of protein fusions in Bacillus subtilis. Gene 227: 101-110.

Leyva-Vazquez,M.A., and Setlow,P. (1994) Cloning and nucleotide sequences of the genes encoding triose phosphate isomerase, phosphoglycerate mutase, and enolase from Bacillus subtilis. J Bacteriol 176: 3903-3910.

Lindner,C., Galinier,A., Hecker,M., and Deutscher,J. (1999) Regulation of the activity of the Bacillus subtilis antiterminator LicT by multiple PEP-dependent, enzyme I- and HPrcatalysed phosphorylation. Mol Microbiol 31: 995-1006.

Lindner,C., Stülke,J., and Hecker,M. (1994) Regulation of xylanolytic enzymes in Bacillus subtilis. Microbiology 140 ( Pt 4): 753-757.

Lorca,G.L., Chung,Y.J., Barabote,R.D., Weyler,W., Schilling,C.H., and Saier,M.H., Jr. (2005) Catabolite repression and activation in Bacillus subtilis: dependency on CcpA, HPr, and HprK. J Bacteriol 187: 7826-7839. 
Ludwig,H., Homuth,G., Schmalisch,M., Dyka,F.M., Hecker,M., and Stülke,J. (2001) Transcription of glycolytic genes and operons in Bacillus subtilis: evidence for the presence of multiple levels of control of the gapA operon. Mol Microbiol 41: 409-422.

Ludwig,H., Rebhan,N., Blencke,H.M., Merzbacher,M., and Stülke,J. (2002) Control of the glycolytic gapA operon by the catabolite control protein A in Bacillus subtilis: a novel mechanism of CcpA-mediated regulation. Mol Microbiol 45: 543-553.

Lulko,A.T., Buist,G., Kok,J., and Kuipers,O.P. (2007) Transcriptome analysis of temporal regulation of carbon metabolism by CcpA in Bacillus subtilis reveals additional target genes. J Mol Microbiol Biotechnol 12: 82-95.

Madec,E., Laszkiewicz,A., Iwanicki,A., Obuchowski,M., and Seror,S. (2002) Characterization of a membrane-linked Ser/Thr protein kinase in Bacillus subtilis, implicated in developmental processes. Mol Microbiol 46: 571-586.

Mann,N.H. (1994) Protein phosphorylation in cyanobacteria. Microbiology 140: 3207-3215.

Marquez,J.A., Hasenbein,S., Koch,B., Fieulaine,S., Nessler,S., Russell,R.B. et al. (2002) Structure of the full-length HPr kinase/phosphatase from Staphylococcus xylosus at 1.95 A resolution: Mimicking the product/substrate of the phospho transfer reactions. Proc Natl Acad Sci U S A 99: 3458-3463.

Martin-Verstraete,I., Deutscher,J., and Galinier,A. (1999) Phosphorylation of HPr and Crh by HprK, early steps in the catabolite repression signalling pathway for the Bacillus subtilis levanase operon. J Bacteriol 181: 2966-2969.

Martin-Verstraete,I., Stülke,J., Klier,A., and Rapoport,G. (1995) Two different mechanisms mediate catabolite repression of the Bacillus subtilis levanase operon. J Bacteriol 177: 69196927.

Martinez-Antonio,A., and Collado-Vides,J. (2003) Identifying global regulators in transcriptional regulatory networks in bacteria. Curr Opin Microbiol 6: 482-489.

Mascher,T., Helmann,J.D., and Unden,G. (2006) Stimulus perception in bacterial signaltransducing histidine kinases. Microbiol Mol Biol Rev 70: 910-938.

Mason,P.W., Carbone,D.P., Cushman,R.A., and Waggoner,A.S. (1981) The importance of inorganic phosphate in regulation of energy metabolism of Streptococcus lactis. J Biol Chem 256: 1861-1866.

Matsumoto,A., Hong,S.K., Ishizuka,H., Horinouchi,S., and Beppu,T. (1994) Phosphorylation of the AfsR protein involved in secondary metabolism in Streptomyces species by a eukaryotic-type protein kinase. Gene 146: 47-56.

Meadow,N.D., Fox,D.K., and Roseman,S. (1990) The bacterial phosphoenolpyruvate: glycose phosphotransferase system. Annu Rev Biochem 59: 497-542.

Meyer,K., Leube,M.P., and Grill,E. (1994) A protein phosphatase 2C involved in ABA signal transduction in Arabidopsis thaliana. Science 264: 1452-1455. 
Mijakovic,I., Poncet,S., Galinier,A., Monedero,V., Fieulaine,S., Janin,J. et al. (2002) Pyrophosphate-producing protein dephosphorylation by HPr kinase/phosphorylase: a relic of early life? Proc Natl Acad Sci U S A 99: 13442-13447.

Min,K.T., Hilditch,C.M., Diederich,B., Errington,J., and Yudkin,M.D. (1993) Sigma F, the first compartment-specific transcription factor of B. subtilis, is regulated by an anti-sigma factor that is also a protein kinase. Cell 74: 735-742.

Missiakas,D., and Raina,S. (1997) Signal transduction pathways in response to protein misfolding in the extracytoplasmic compartments of E. coli: role of two new phosphoprotein phosphatases PrpA and PrpB. EMBO J 16: 1670-1685.

Miwa,Y., Nagura,K., Eguchi,S., Fukuda,H., Deutscher,J., and Fujita,Y. (1997) Catabolite repression of the Bacillus subtilis gnt operon exerted by two catabolite-responsive elements. Mol Microbiol 23: 1203-1213.

Monedero,V., Poncet,S., Mijakovic,I., Fieulaine,S., Dossonnet,V., Martin-Verstraete,I. et al. (2001) Mutations lowering the phosphatase activity of HPr kinase/phosphatase switch off carbon metabolism. EMBO J 20: 3928-3937.

Monod,J. (1942) Recherches sur la Croissance des Cultures Bacteriennes. Thesis, Hermann et Cie, Paris.

Moreno,M.S., Schneider,B.L., Maile,R.R., Weyler,W., and Saier,M.H., Jr. (2001) Catabolite repression mediated by the CcpA protein in Bacillus subtilis: novel modes of regulation revealed by whole-genome analyses. Mol Microbiol 39: 1366-1381.

Neves,A.R., Ramos,A., Nunes,M.C., Kleerebezem,M., Hugenholtz,J., de Vos,W.M. et al. (1999) In vivo nuclear magnetic resonance studies of glycolytic kinetics in Lactococcus lactis. Biotechnol Bioeng 64: 200-212.

Nguyen,H.D., Nguyen,Q.A., Ferreira,R.C., Ferreira,L.C., Tran,L.T., and Schumann,W. (2005) Construction of plasmid-based expression vectors for Bacillus subtilis exhibiting full structural stability. Plasmid 54: 241-248.

Nihashi,J., and Fujita,Y. (1984) Catabolite repression of inositol dehydrogenase and gluconate kinase syntheses in Bacillus subtilis. Biochim Biophys Acta 798: 88-95.

Nystrom,T. (1994) The glucose-starvation stimulon of Escherichia coli: induced and repressed synthesis of enzymes of central metabolic pathways and role of acetyl phosphate in gene expression and starvation survival. Mol Microbiol 12: 833-843.

Obuchowski,M. (2005) [Serine-threonine protein phosphatases from Bacillus subtilis]. Postepy Biochem 51: 95-104.

Obuchowski,M., Madec,E., Delattre,D., Boel,G., Iwanicki,A., Foulger,D., and Seror,S.J. (2000) Characterization of PrpC from Bacillus subtilis, a member of the PPM phosphatase family. $J$ Bacteriol 182: 5634-5638.

Pan,Q., Garsin,D.A., and Losick,R. (2001) Self-reinforcing activation of a cell-specific transcription factor by proteolysis of an anti-sigma factor in B. subtilis. Mol Cell 8: 873-883. 
Pané-Farré J, Lewis RJ, Stülke J. (2005) The RsbRST stress module in bacteria: a signalling system that may interact with different output modules. J Mol Microbiol Biotechnol 9: 65-76.

Pardee,A.B., Jacob,F., and Monod,J. (1959) The genetic control and cytoplasmic expression of "inducibility" in the synthesis of $\beta$-galactosidase by E. coli. J Mol Biol 1: 165-178.

Pettigrew,D.W., Ma,D.P., Conrad,C.A., and Johnson,J.R. (1988) Escherichia coli glycerol kinase. Cloning and sequencing of the glpK gene and the primary structure of the enzyme. $J$ Biol Chem 263: 135-139.

Pompeo,F., Luciano,J., and Galinier,A. (2007) Interaction of GapA with HPr and Its Homologue, Crh: Novel Levels of Regulation of a Key Step of Glycolysis in Bacillus subtilis? J Bacteriol 189: 1154-7.

Poncet,S., Mijakovic,I., Nessler,S., Gueguen-Chaignon,V., Chaptal,V., Galinier,A. et al. (2004) HPr kinase/phosphorylase, a Walker motif A-containing bifunctional sensor enzyme controlling catabolite repression in Gram-positive bacteria. Biochim Biophys Acta 1697: 123135 .

Postma,P.W., Lengeler,J.W., and Jacobson,G.R. (1993) Phosphoenolpyruvate:carbohydrate phosphotransferase systems of bacteria. Microbiol Rev 57: 543-594.

Presecan-Siedel,E., Galinier,A., Longin,R., Deutscher,J., Danchin,A., Glaser,P., and MartinVerstraete,I. (1999) Catabolite regulation of the pta gene as part of carbon flow pathways in Bacillus subtilis. J Bacteriol 181: 6889-6897.

Ramström,H., Sanglier,S., Leize-Wagner,E., Philippe,C., Van Dorsselaer,A., and Haiech,J. (2003) Properties and regulation of the bifunctional enzyme HPr kinase/phosphatase in Bacillus subtilis. J Biol Chem 278: 1174-1185.

Reizer,J., Bergstedt,U., Galinier,A., Küster,E., Saier,M.H., Jr., Hillen,W. et al. (1996) Catabolite repression resistance of gnt operon expression in Bacillus subtilis conferred by mutation of His-15, the site of phosphoenolpyruvate-dependent phosphorylation of the phosphocarrier protein HPr. J Bacteriol 178: 5480-5486.

Reizer,J., Hoischen,C., Titgemeyer,F., Rivolta,C., Rabus,R., Stülke,J. et al. (1998) A novel protein kinase that controls carbon catabolite repression in bacteria. Mol Microbiol 27: 11571169.

Reizer,J., Novotny,M.J., Stuiver,I., and Saier,M.H., Jr. (1984) Regulation of glycerol uptake by the phosphoenolpyruvate-sugar phosphotransferase system in Bacillus subtilis. J Bacteriol 159: $243-250$.

Reizer,J., Bachem,S., Reizer,A., Arnaud, M., Saier,M.H., Jr., and Stülke, J. (1999) Novel phosphotransferase system genes revealed by genome analysis-the complete complement of PTS proteins encoded within the genome of Bacillus subtilis. Microbiology 145:3419-3429.

Reizer,J., Sutrina,S.L., Saier,M.H., Stewart,G.C., Peterkofsky,A., and Reddy,P. (1989) Mechanistic and physiological consequences of $\operatorname{HPr}($ ser) phosphorylation on the activities of the phosphoenolpyruvate:sugar phosphotransferase system in gram-positive bacteria: studies with site-specific mutants of HPr. Embo J 8: 2111-20. 
Reizer,J., Sutrina,S.L., Wu,L.F., Deutscher,J., Reddy,P., and Saier,M.H., Jr. (1992) Functional interactions between proteins of the phosphoenolpyruvate:sugar phosphotransferase systems of Bacillus subtilis and Escherichia coli. J Biol Chem 267: 91589169.

Rokop,M.E., Auchtung,J.M., and Grossman,A.D. (2004) Control of DNA replication initiation by recruitment of an essential initiation protein to the membrane of Bacillus subtilis. Mol Microbiol 52: 1757-1767.

Rutberg,B. (1997) Antitermination of transcription of catabolic operons. Mol Microbiol 23: 413-421.

Saier,M.H., Jr. (1993) Introduction: protein phosphorylation and signal transduction in bacteria. J Cell Biochem 51: 1-6.

Saier,M.H., Jr., Goldman,S.R., Maile,R,R., Moreno,M,S., Weyler,W., Yang,N., and Paulsen,I,T (2002) Overall transport capabilities of Bacillus subtilis. In: A. L Sonenshein, J. A. Hoch, and R. Losick (ed.), Bacillus subtilis and its closest relatives. ASM press, Washington, D.C. p. 113-128.

Saier,M.H., Jr., and Roseman,S. (1976) Sugar transport. Inducer exclusion and regulation of the melibiose, maltose, glycerol, and lactose transport systems by the phosphoenolpyruvate:sugar phosphotransferase system. J Biol Chem 251: 6606-6615.

Sambrook, J., Fritsch. E.F., and Maniatis, T (1989) Molecular cloning: a laboratory manual, Cold Spring Harbour Laboratory. Cold Spring Harbour, N.Y.

Sambrook,J., and Russell,D. (2001) Molecular Cloning: A Laboratory Manual, Cold Spring Harbor Laboratory. Cold Spring Harbour, N.Y.

Schmalisch,M.H. (2004) Regulation der Glucoseaufnahme and -verwertung in Bacillus subtilis durch reversible Proteinphosphorylierungen. Phd thesis. University of Erlangen.

Schmalisch,M.H., Bachem,S., and Stülke,J. (2003) Control of the Bacillus subtilis antiterminator protein GlcT by phosphorylation. Elucidation of the phosphorylation chain leading to inactivation of GlcT. J Biol Chem 278: 51108-51115.

Schmiedel,D., and Hillen,W. (1996a) A Bacillus subtilis 168 mutant with increased xylose uptake can utilize xylose as sole carbon source. FEMS Microbiol Lett 135: 175-178.

Schmiedel,D., and Hillen,W. (1996b) Contributions of XylR, CcpA and cre to diauxic growth of Bacillus megaterium and to xylose isomerase expression in the presence of glucose and xylose. Mol Gen Genet 250: 259-266.

Schumacher,M.A., Allen,G.S., Diel,M., Seidel,G., Hillen,W., and Brennan,R.G. (2004) Structural basis for allosteric control of the transcription regulator CcpA by the phosphoprotein HPr-Ser46-P. Cell 118: 731-741.

Schumacher,M.A., Seidel,G., Hillen,W., and Brennan,R.G. (2006) Phosphoprotein CrhSer46-P displays altered binding to CcpA to effect carbon catabolite regulation. J Biol Chem 281: 6793-6800. 
Schumacher,M.A., Seidel,G., Hillen,W., and Brennan,R.G. (2007) Structural mechanism for the fine-tuning of CcpA function by the small molecule effectors glucose 6-phosphate and fructose 1,6-bisphosphate. J Mol Biol 368: 1042-1050.

Seidel,G., Diel,M., Fuchsbauer,N., and Hillen,W. (2005) Quantitative interdependence of coeffectors, CcpA and cre in carbon catabolite regulation of Bacillus subtilis. Febs J 272: 2566-2577.

Shi,L., Potts,M., and Kennelly,P.J. (1998) The serine, threonine, and/or tyrosine-specific protein kinases and protein phosphatases of prokaryotic organisms: a family portrait. FEMS Microbiol Rev 22: 229-253.

Singh,K.D., Halbedel,S., Görke,B., and Stülke,J. (2007) Control of the phosphorylation state of the HPr protein of the phosphotransferase system in Bacillus subtilis: implication of the protein phosphatase PrpC. J Mol Microbiol Biotechnol 13: 165-171.

Singh,K.D., Schmalisch,M.H., Stülke,J., and Görke,B. (2008) Carbon Catabolite Repression in Bacillus subtilis: A Quantitative Analysis of Repression Exerted by Different Carbon Sources. J Bacteriol. DOI: 10.1128/JB.00848-08

Skarlatos,P., and Dahl,M.K. (1998) The glucose kinase of Bacillus subtilis. J Bacteriol 180: 3222-3226.

Smith,C.A. (2006) Structure, function and dynamics in the mur family of bacterial cell wall ligases. J Mol Biol 362: 640-655.

Sprang,S.R., Acharya,K.R., Goldsmith,E.J., Stuart,D.I., Varvill,K., Fletterick,R.J. et al. (1988) Structural changes in glycogen phosphorylase induced by phosphorylation. Nature 336: $215-221$.

Srivatsan,A., Han,Y., Peng,J., Tehranchi,A.K., Gibbs,R., Wang,J.D., and Chen,R. (2008) High-precision, whole-genome sequencing of laboratory strains facilitates genetic studies. PLoS Genet 4: e1000139.

Steinmetz,M. (1993) Carbohydrate metabolism: pathways, enzymes, genetic regulation, and evolution. In: A. L Sonenshein, J. A. Hoch, and R. Losick (ed.), Bacillus subtilis and other Gram positive bacteria: Biochemistry, Physiology, and Molecular Genetics. ASM press, Washington, D.C. p. 157-170.

Stock,A.M., Robinson,V.L., and Goudreau,P.N. (2000) Two-component signal transduction. Annu Rev Biochem 69: 183-215.

Stock,J.B., Stock,A.M., and Mottonen,J.M. (1990) Signal transduction in bacteria. Nature 344: 395-400.

Stock,J.B., Surette,M.G., McCleary,W.R., and Stock,A.M. (1992) Signal transduction in bacterial chemotaxis. J Biol Chem 267: 19753-19756.

Strauch,M.A. (1995) AbrB modulates expression and catabolite repression of a Bacillus subtilis ribose transport operon. J Bacteriol 177: 6727-6731. 
Stülke,J., Arnaud,M., Rapoport,G., and Martin-Verstraete,I. (1998) PRD--a protein domain involved in PTS-dependent induction and carbon catabolite repression of catabolic operons in bacteria. Mol Microbiol 28: 865-874.

Stülke,J., and Hillen,W. (2000) Regulation of carbon catabolism in Bacillus species. Annu Rev Microbiol 54: 849-880.

Stülke,J., Martin-Verstraete,I., Zagorec,M., Rose,M., Klier,A., and Rapoport,G. (1997) Induction of the Bacillus subtilis ptsGHI operon by glucose is controlled by a novel antiterminator, GlcT. Mol Microbiol 25: 65-78.

Teresa,P.M., Felisa,N.M., Aguilar,J., Badia,J., and Baldoma,L. (2003) Role of 2phosphoglycolate phosphatase of Escherichia coli in metabolism of the 2-phosphoglycolate formed in DNA repair. J Bacteriol 185: 5815-5821.

Thevenot,T., Brochu,D., Vadeboncoeur,C., and Hamilton,I.R. (1995) Regulation of ATPdependent P-(Ser)-HPr formation in Streptococcus mutans and Streptococcus salivarius. $J$ Bacteriol 177: 2751-2759.

Tortosa,P., Aymerich,S., Linder,C., Saier,M.H., Jr., and Coq Le,D. (1997) Multiple phosphorylation of SacY, Bacillus subtilis transcriptional antiterminator negatively controlled by the phosphotransferase system. J Biol Chem 272: 17230-17237.

Thompson,J., and Torchia,D.A. (1984) Use of 31P nuclear magnetic resonance spectroscopy and 14C fluorography in studies of glycolysis and regulation of pyruvate kinase in Streptococcus lactis. J Bacteriol 158: 791-800.

Unden,G., and Bongaerts,J. (1997) Alternative respiratory pathways of Escherichia coli: energetics and transcriptional regulation in response to electron acceptors. Biochim Biophys Acta 1320: 217-234.

Vassylyev,D.G., and Symersky,J. (2007) Crystal structure of pyruvate dehydrogenase phosphatase 1 and its functional implications. J Mol Biol 370: 417-426.

Viana,R., Monedero,V., Dossonnet,V., Vadeboncoeur,C., Perez-Martinez,G., and Deutscher,J. (2000) Enzyme I and HPr from Lactobacillus casei: their role in sugar transport, carbon catabolite repression and inducer exclusion. Mol Microbiol 36: 570-584.

Voskuil,M.I., and Chambliss,G.H. (1996) Significance of HPr in catabolite repression of alpha-amylase. J Bacteriol 178: 7014-7015.

Wach,A. (1996) PCR-synthesis of marker cassettes with long flanking homology regions for gene disruptions in S. cerevisiae. Yeast 12: 259-265.

Warner,J.B., Krom,B.P., Magni,C., Konings,W.N., and Lolkema,J.S. (2000) Catabolite repression and induction of the $\mathrm{Mg}(2+)$-citrate transporter CitM of Bacillus subtilis. $J$ Bacteriol 182: 6099-6105.

Warner,J.B., and Lolkema,J.S. (2003) CcpA-dependent carbon catabolite repression in bacteria. Microbiol Mol Biol Rev 67: 475-490. 
Watanabe,S., Hamano,M., Kakeshita,H., Bunai,K., Tojo,S., Yamaguchi,H. et al. (2003) Mannitol-1-phosphate dehydrogenase (MtID) is required for mannitol and glucitol assimilation in Bacillus subtilis: possible cooperation of mtl and gut operons. J Bacteriol 185: 4816-4824.

Weickert,M.J., and Chambliss,G.H. (1990) Site-directed mutagenesis of a catabolite repression operator sequence in Bacillus subtilis. Proc Natl Acad Sci U S A 87: 6238-6242.

Weinrauch,Y., Msadek,T., Kunst,F., and Dubnau,D. (1991) Sequence and properties of comQ, a new competence regulatory gene of Bacillus subtilis. J Bacteriol 173: 5685-5693.

Yang,Q.H., Zhu,Z., Dong,M.Q., Ling,S., Wu,C.L., and Li,L. (2001) Binding of ATP to the fructose-2,6-bisphosphatase domain of chicken liver 6-phosphofructo-2-kinase/fructose-2,6bisphosphatase leads to activation of its 6-phosphofructo-2-kinase. J Biol Chem 276: 2460824613.

Yang,X., Kang,C.M., Brody,M.S., and Price,C.W. (1996) Opposing pairs of serine protein kinases and phosphatases transmit signals of environmental stress to activate a bacterial transcription factor. Genes Dev 10: 2265-2275.

Ye,J.J., and Saier,M.H., Jr. (1995) Cooperative binding of lactose and the phosphorylated phosphocarrier protein $\mathrm{HPr}(\mathrm{Ser}-\mathrm{P})$ to the lactose/H+ symport permease of Lactobacillus brevis. Proc Natl Acad Sci U S A 92: 417-421.

Ye,J.J., and Saier,M.H., Jr. (1996) Regulation of sugar uptake via the phosphoenolpyruvatedependent phosphotransferase systems in Bacillus subtilis and Lactococcus lactis is mediated by ATP-dependent phosphorylation of seryl residue 46 in HPr. J Bacteriol 178: 3557-3563.

Ye,R., and Wong,S.L. (1994) Transcriptional regulation of the Bacillus subtilis glucitol dehydrogenase gene. J Bacteriol 176: 3314-3320.

Yoshida,K., Kobayashi,K., Miwa,Y., Kang,C.M., Matsunaga,M., Yamaguchi,H. et al. (2001) Combined transcriptome and proteome analysis as a powerful approach to study genes under glucose repression in Bacillus subtilis. Nucleic Acids Res 29: 683-692.

Yoshida,T., Cai,S., and Inouye,M. (2002) Interaction of EnvZ, a sensory histidine kinase, with phosphorylated OmpR, the cognate response regulator. Mol Microbiol 46: 1283-1294.

Zalieckas,J.M., Wray,L.V., Jr., and Fisher,S.H. (1999) trans-acting factors affecting carbon catabolite repression of the hut operon in Bacillus subtilis. J Bacteriol 181: 2883-2888.

Zorrilla,S., Doan,T., Alfonso,C., Margeat,E., Ortega,A., Rivas,G. et al. (2007) Inducermodulated cooperative binding of the tetrameric CggR repressor to operator DNA. Biophys $J$ 92: $3215-3227$. 


\section{Supplementary material}

\section{Oligonucleotides}

Table. 11. Oligonucleotides

\begin{tabular}{|c|c|c|}
\hline Name & Sequence $5^{\prime} \rightarrow 3^{, \#}$ & Description \\
\hline SK1 & $\begin{array}{l}\text { CGTCAGGCCTATGGTTCAACAGAAAGTG } \\
\text { GAAGT }\end{array}$ & $\begin{array}{l}\text { Cloning of B. subtilis crh in pDG148 } \\
\text { (fwd) (StuI) }\end{array}$ \\
\hline SK2 & $\begin{array}{l}\text { CGTCAGGCCTCTAAACTTCTTCTTGAACG } \\
\text { TAAG }\end{array}$ & $\begin{array}{l}\text { Cloning of B. subtilis crh in pDG148 } \\
\text { (rev) (StuI) }\end{array}$ \\
\hline SK3 & $\begin{array}{l}\text { GGCGAATTCGTGTAACAATTTTGATCAG } \\
\text { TCCG }\end{array}$ & $\begin{array}{l}\text { Cloning of B. subtilis hprK with } 700 \\
\text { bp upstream of the start codon ( to } \\
\text { include the putative promoter region) } \\
\text { (fwd) (EcoRI) }\end{array}$ \\
\hline SK4 & $\begin{array}{l}\text { GGCGAGCTCTATTCTTCTTGTTCACCGTC } \\
\text { TT }\end{array}$ & $\begin{array}{l}\text { Cloning of B. subtilis hprK (rev) } \\
(\text { SacI) }\end{array}$ \\
\hline SK5 & GTAAACTACGTGCCTGAACG & Amplification of B. subtilis prpC \\
\hline SK6 & CCGCCGCCTATGACGCG & Amplification of $B$. subtilis prpC \\
\hline SK7 & TGTTTTGGACTGAGACACTG & $\begin{array}{l}\text { Amplification of regulatory region } \\
\text { upstream of } B \text {. subtilis xynP }\end{array}$ \\
\hline SK8 & CTTGTGGACAAAGCCAATCAGA & Amplification of $B$. subtilis prkC \\
\hline SK9 & ATTCATCTTTCGGATACTCAATG & Amplification of B. subtilis prkC \\
\hline SK10 & ACGTAATCACCGACAAGAGGGGTA & Amplification of B. subtilis prkC \\
\hline SK11 & $\begin{array}{l}\text { GGCGGATCCGTGGCAAAGGTTCGCACAA } \\
\text { AAGA }\end{array}$ & $\begin{array}{l}\text { Cloning of B. subtilis hprK in } \\
\text { pGP380 (fwd) (BamHI) }\end{array}$ \\
\hline SK12 & $\begin{array}{l}\text { CGTCCCCGGGCTATTCTTCTTGTTCACCG } \\
\text { TCTT }\end{array}$ & $\begin{array}{l}\text { Cloning of B. subtilis hprK (rev) } \\
(X m a \mathrm{I})\end{array}$ \\
\hline SK13 & ACGCTTATTAATACGAATGAATTA & $\begin{array}{l}\text { Amplification of B. subtilis yvoE } \\
\text { (fwd) for RNA probe }\end{array}$ \\
\hline SK14 & $\begin{array}{l}\text { CTAATACGACTCACTATAGGGAGACTCATT } \\
\text { TTCTCAAGCATAAA }\end{array}$ & $\begin{array}{l}\text { Amplification of B. subtilis yvoE for } \\
\text { RNA probe }\end{array}$ \\
\hline SK15 & $\begin{array}{l}\text { GGCGGATCCATGAGTGACAAACAAGTA } \\
\text { ACGACG }\end{array}$ & $\begin{array}{l}\text { Cloning of B. subtilis yvoE in } \\
\text { pHCMC05 (fwd) (BamHI) }\end{array}$ \\
\hline SK16 & $\begin{array}{l}\text { CGTCCCCGGGTTACTTCACTCCAACGATT } \\
\text { TGTAA }\end{array}$ & $\begin{array}{l}\text { Cloning of B. subtilis yvoE in } \\
\text { pHCMC05 (rev) (XmaI) }\end{array}$ \\
\hline SK17 & $\begin{array}{l}\text { GGCGGATCCTGAATGAAGCGATAGAACC } \\
\text { ACTC }\end{array}$ & $\begin{array}{l}\text { Cloning of } B . \text { subtilis lgt-yvoF in } \\
\text { pHCMC } 05 \text { (fwd) (BamHI) }\end{array}$ \\
\hline SK18 & $\begin{array}{l}\text { CGTCTCTAGATTATTCAGCGGACTTTTTC } \\
\text { AATC }\end{array}$ & $\begin{array}{l}\text { Cloning of } B . \text { subtilis lgt-yvoF in } \\
\text { pHCMC05 (rev) (XbaI) }\end{array}$ \\
\hline SK19 & GTGGCAAAGGTTCGCACAAAAGACGT & $\begin{array}{l}\text { Knockout of } B . \text { subtilis lgt-yvoF with } \\
\text { LFH-PCR*(up fwd). Complementary } \\
\text { to } h p r K\end{array}$ \\
\hline
\end{tabular}




\begin{tabular}{|c|c|c|}
\hline Name & Sequence 5 , $\rightarrow 3$, \# & Description \\
\hline SK20 & $\begin{array}{l}\text { CCTATCACCTCAAATGGTTCGCTGAAC } \\
\text { GCCAАCTCСТАТTCTTCTTG }\end{array}$ & $\begin{array}{l}\text { Knockout of B. subtilis lgt-yvoF with } \\
\text { LFH-PCR (up rev). Sequence in } \\
\text { bold is complementary to the forward } \\
\text { primer of kanamycin cassette }\end{array}$ \\
\hline SK21 & $\begin{array}{l}\text { CTGAGCAAATACGACAATATTCATGCTT } \\
\text { TT }\end{array}$ & $\begin{array}{l}\text { Knockout of } B \text {. subtilis lgt-yvoF } \\
\text { with LFH-PCR* (down rev) }\end{array}$ \\
\hline SK22 & $\begin{array}{l}\text { CGAGCGCCTACGAGGAATTTGTATCG } \\
\text { CATCAGCGGACTTTTTTTGTTAAAATT }\end{array}$ & $\begin{array}{l}\text { Knockout of B. subtilis lgt-yvoF } \\
\text { with LFH-PCR }{ }^{*} \text { (down fwd) } \\
\text { Sequence in bold is complementary } \\
\text { to the reverse primer of kanamycin } \\
\text { cassette }\end{array}$ \\
\hline SK23 & ATGAAGAAGATCTTTCTGGCCGGTC & $\begin{array}{l}\text { Knockout of B. subtilis yvoE with } \\
\text { LFH-PCR (upfwd). Complementary } \\
\text { to } y v o D\end{array}$ \\
\hline SK24 & $\begin{array}{l}\text { CCTATCACCTCAAATGGTTCGCTGTCA } \\
\text { TATTGCTTCCTTTCCAACCGC }\end{array}$ & $\begin{array}{l}\text { Knockout of B. subtilis yvoE with } \\
\text { LFH-PCR* (up rev). Complementary } \\
\text { to } y v o E \text {. Sequence in bold is } \\
\text { complementary to the forward primer } \\
\text { of kanamycin cassette }\end{array}$ \\
\hline
\end{tabular}

SK25 CGAGCGCCTACGAGGAATTTGTATCG GTGAGAAAAACAGATCGTCATCCGGTCT CG

ATGGGAAGATGTTGGTGAGGATGCG

GGCCTCGAGGTTCTTTCAATTCATGACC GGGCTTGCC

Knockout of B. subtilis yvoE with LFH-PCR ${ }^{*}$ (down fwd). Sequence in bold is complementary to the reverse primer of kanamycin cassette

Knockout of B. subtilis yvoE with LFH-PCR ${ }^{*}$ (down rev)

Cloning of the region $(-279$ to +86 , transcription start site as +1$)$ upstream of $x y n P$ gene in pDG148 $(A v a \mathrm{I})$

SK28 GGCGGATCCCTCTTATCCCCCTTCCATCC ATATT

Cloning of the region $(-279-+86$, transcription start site as +1 ) upstream of $x y n P$ gene in pDG148 (BamHI)

SK29 GGCGGATCCCAAGCAAAATCTCCAGACG CATATCCAACT

Cloning of the region (-279 to+380, transcription start site as +1 ) upstream of $x y n P$ gene in pDG148 (BamHI)

SK30 CGTCAGGCCTATGAGCAATATTACGATC TACGATGTAGCG

SK31 CGTCAGGCCTTTTCTTATGACTTGGTTGA CTTTCTAAGC

Cloning of B. subtilis ccpA in pDG148 (fwd) (StuI)

Cloning of B. subtilis ccpA in pDG148 (rev) (StuI)

SK32 GGCGGATCCATGGTTCAACAGAAAGTGG AAGTTCG

SK33 GGCCTGCAGCTAAACTTCTTCTTGAACG TAAGCAGCC

SK34 GGCGGATCCATGAGCAATATTACGATCT ACGATGTA

Cloning of B. subtilis crh in pGP380 (fwd) (BamHI)

Cloning of B. subtilis crh in pGP380 (rev) (PstI)

Cloning of B. subtilis ccpA in pGP380 (fwd) (BamHI) 


\begin{tabular}{|c|c|c|}
\hline Name & Sequence 5 , $\rightarrow 3$, \# & Description \\
\hline SK35 & $\begin{array}{l}\text { GGCCGTCGACTTTTCTTATGACTTGGTTG } \\
\text { ACTTTCTAAGC }\end{array}$ & $\begin{array}{l}\text { Cloning of B. subtilis ccpA in } \\
\text { pGP380 (rev) (SalI) }\end{array}$ \\
\hline SK36 & $\begin{array}{l}\text { GCCTCGTATGTTTCAACCATTTGTTCCAG } \\
\text { G }\end{array}$ & $\begin{array}{l}\text { Sequencing of inserts cloned in } \\
\text { pHCMC05 }\end{array}$ \\
\hline SK37 & $\begin{array}{l}\text { GGCCTGCAGAACTTCTTCTTGAACGTAA } \\
\text { GCAGCC }\end{array}$ & $\begin{array}{l}\text { Cloning of B. subtilis crh in pGP382 } \\
\text { (rev) (PstI) }\end{array}$ \\
\hline SK38 & $\begin{array}{l}\text { ATGAATGAAGCGATAGAACCACTCAATC } \\
\text { CGATAGCAT }\end{array}$ & $\begin{array}{l}\text { Knockout of B. subtilis yvoD-yvoF } \\
\text { with LFH-PCR* (up fwd) }\end{array}$ \\
\hline SK39 & $\begin{array}{l}\text { CCTATCACCTCAAATGGTTCGCTGGTC } \\
\text { TACTCCGCGTACCGCTCCTTCGAGTATC }\end{array}$ & $\begin{array}{l}\text { Knockout of B. subtilis yvoD-yvoF } \\
\text { with LFH-PCR }{ }^{*} \text {. Sequence in bold is } \\
\text { complementary to the forward primer } \\
\text { of kanamycin cassette (up rev) }\end{array}$ \\
\hline SK40 & $\begin{array}{l}\text { TCTCAATGATCCGTATGGCTATAAAAAC } \\
\text { AG }\end{array}$ & Sequencing of $B$. subtilis yvoF \\
\hline SK41 & $\begin{array}{l}\text { AATCGTATTGATTGTTTTGGCTGTGGCAG } \\
\text { C }\end{array}$ & Sequencing of B. subtilis yvoD \\
\hline SK42 & $\begin{array}{l}\text { CGTCAGGCCTGTGGCAAAGGTTCGCACA } \\
\text { AAAGACGTA }\end{array}$ & $\begin{array}{l}\text { Cloning of B. subtilis hprK in } \\
\text { pDG148 (fwd) (StuI) }\end{array}$ \\
\hline SK43 & $\begin{array}{l}\text { CGTCAGGCCTCTATTCTTCTTGTTCACCG } \\
\text { TCTTCAATGAC }\end{array}$ & $\begin{array}{l}\text { Cloning of B. subtilis hprK in } \\
\text { pDG148 (rev) (StuI) }\end{array}$ \\
\hline SK44 & GTTCTACATCCAGAACAACCTCTG & $\begin{array}{l}\text { Sequencing of inserts cloned in } \\
\text { pDG148 }\end{array}$ \\
\hline SK45 & GCAACCGTTTTTTCGGAAGGAAATG & $\begin{array}{l}\text { Sequencing of inserts cloned in } \\
\text { pDG148 }\end{array}$ \\
\hline SK46 & $\begin{array}{l}\text { GCCAAGCTTGTTAAAAGGAGAATGATA } \\
\text { AAAATGGCACAA }\end{array}$ & $\begin{array}{l}\text { Cloning of } B . \text { subtilis pts } H \text { in pdr } 79 . \\
\text { Sequence in bold is the native } \\
\text { ribosome binding site of } p t s H(\mathrm{fwd}) \\
(\text { HindIII) }\end{array}$ \\
\hline SK47 & $\begin{array}{l}\text { GGCGTCGAC } \\
\text { TTACTCGCCGAGTCCTTCGCTTTT. }\end{array}$ & $\begin{array}{l}\text { Cloning of B. subtilis ptsH in pdr79 } \\
\text { (rev) (SalI) }\end{array}$ \\
\hline SK48 & $\begin{array}{l}\text { CCGGGATCCAGATGAGTGTTAGTGAATC } \\
\text { ACATGATATTCA }\end{array}$ & $\begin{array}{l}\text { Cloning of B. subtilis yvcJ in } \\
\text { pSG1729 (fwd) (BamHI) }\end{array}$ \\
\hline SK49 & $\begin{array}{l}\text { CCGGAATTCTTATTTCCGGCTTCTCTTTT } \\
\text { CAATGTC }\end{array}$ & $\begin{array}{l}\text { Cloning of B. subtilis yvcJ in } \\
\text { pSG1729 (rev) (EcoRI) }\end{array}$ \\
\hline SK50 & $\begin{array}{l}\text { CCGGGATCCAGATGGGACAAAAGCCGA } \\
\text { AAATCGCAATCTTT }\end{array}$ & $\begin{array}{l}\text { Cloning of B. subtilis yvcK in } \\
\text { pSG1729 (fwd) (BamHI) }\end{array}$ \\
\hline SK51 & $\begin{array}{l}\text { CCGGAATTCTCATTCTTTCAGTAAATCAA } \\
\text { CAAGAAGAGAGGCC }\end{array}$ & $\begin{array}{l}\text { Cloning of B. subtilis yvcK in } \\
\text { pSG1729 (rev) (EcoRI) }\end{array}$ \\
\hline SK52 & $\begin{array}{l}\text { CCGGGTACCATGGTTCAACAGAAAGTGG } \\
\text { AAGTTC }\end{array}$ & $\begin{array}{l}\text { Cloning of } B \text {. subtilis crh in } \\
\text { pMUTIN-cMyc (fwd) }(K p n I)\end{array}$ \\
\hline SK53 & $\begin{array}{l}\text { CCGATCGATAAGATCTTCTTCGCTAAT } \\
\text { AAGTTTTTGTTCGCCAACTTCTTCTTGA } \\
\text { ACGTAAGCAGCC }\end{array}$ & $\begin{array}{l}\text { Cloning of B. subtilis crh in } \\
\text { pMUTIN-cMyc: Sequence in bold is } \\
\text { c-Myc tag. Introduces myc tag C- } \\
\text { terminally (rev) }\end{array}$ \\
\hline
\end{tabular}




\begin{tabular}{|c|c|c|}
\hline Name & Sequence $5^{\prime} \rightarrow 3^{, \#}$ & Description \\
\hline SK54 & ATTTTATTCCTGGACGCGAA & Sequencing of $B$. subtilis yvcJ \\
\hline SK55 & GCTGAATGTAAGAGGAAAGGTT & Sequencing of $B$. subtilis $y v c K$ \\
\hline SK56 & AАCСТTTCCTCTTACATTCAGCAC & Sequencing of $B$. subtilis $y v c K$ \\
\hline SK57 & $\begin{array}{l}\text { GCCAAGCTTTAAAGAAAAGGGGAGATC } \\
\text { TTATGGTTCAAC }\end{array}$ & $\begin{array}{l}\text { Cloning of } B . \text { subtilis crh in pdr79. } \\
\text { Sequence in bold ist the native } \\
\text { ribosome binding site of } c r h \text { (fwd) } \\
\text { (HindIII) }\end{array}$ \\
\hline SK58 & $\begin{array}{l}\text { GGCGTCGAC } \\
\text { CTAAACTTCTTCTTGAACGTAAGC }\end{array}$ & $\begin{array}{l}\text { Cloning of B. subtilis crh in pdr79 } \\
\text { (rev) (SalI) }\end{array}$ \\
\hline SK59 & $\begin{array}{l}\text { GGCGAGCTCAATGAAAATTGCTTTGATC } \\
\text { GCGCATG }\end{array}$ & $\begin{array}{l}\text { Cloning of } B \text {. subtilis } m g s A \text { in } \\
\text { pGP172 (fwd) (SacI) }\end{array}$ \\
\hline SK60 & $\begin{array}{l}\text { GGCGGATCCTTATACATTCGGCTCTTCTC } \\
\text { CCCGAAGAA }\end{array}$ & $\begin{array}{l}\text { Cloning of } B \text {. subtilis } m g s A \text { in } \\
\text { pGP172 (rev) }(\text { BamHI) }\end{array}$ \\
\hline SK61 & $\begin{array}{l}\text { GGCGTCGACTTATTCAGCGGACTTTTTCA } \\
\text { ATCTTTC }\end{array}$ & $\begin{array}{l}\text { Cloning of } B \text {. subtilis hprK operon in } \\
\text { pGP380 (rev) (SalI) }\end{array}$ \\
\hline SK62 & $\begin{array}{l}\text { CAGGAATTCTCATGTTTGACAGCTTATC } \\
\text { ATCGGCA }\end{array}$ & $\begin{array}{l}\text { Amplification of cat cassette from } \\
\text { pBGM35 (EcoRI) }\end{array}$ \\
\hline SK63 & $\begin{array}{l}\text { GGCGAATTCTTATAAAAGCCAGTCATTA } \\
\text { GGCCTA }\end{array}$ & $\begin{array}{l}\text { Amplification of cat cassette from } \\
\text { pBGM35 (EcoRI) }\end{array}$ \\
\hline KS12 & $\begin{array}{l}\text { AAAAAGCTTGGTTCTATCGCTTCATTCAT } \\
\text { TTAACGC }\end{array}$ & $\begin{array}{l}\text { Cloning of B. subtilis hprK in } \\
\text { pGP380 (HindIII) }\end{array}$ \\
\hline $\begin{array}{l}\text { pUC } \\
\text { fwd }\end{array}$ & GTAAAACGACGGCCAGTG & $\begin{array}{l}\text { Sequencing of inserts cloned in } \\
\text { pGP380 }\end{array}$ \\
\hline $\begin{array}{l}\mathrm{pUC} \\
\mathrm{rev}\end{array}$ & GGAAACAGCTATGACCATG & $\begin{array}{l}\text { Sequencing of inserts cloned in } \\
\text { pGP380 }\end{array}$ \\
\hline NR1 & CCTTTGTCAAAAAAGTAAATCAAAAG & Amplification of B. subtilis xylR \\
\hline BG94 & CTACAAGGTGTGGCATAATG & $\begin{array}{l}\text { Sequencing of inserts cloned in } \\
\text { pDG148 }\end{array}$ \\
\hline Kan fwd & CAGCGAACCATTTGAGGTGATAGG & $\begin{array}{l}\text { Amplification of kan cassette from } \\
\text { pDG780 }\end{array}$ \\
\hline Kan rev & CGATACAAATTCCTCGTAGGCGCTCGG & $\begin{array}{l}\text { Amplification of kan cassette from } \\
\text { pDG780 }\end{array}$ \\
\hline $\begin{array}{l}\text { Kan } \\
\text { checkfwd }\end{array}$ & CATCCGCAACTGTCCATACTCTG & $\begin{array}{l}\text { Sequencing of kan cassette amplified } \\
\text { from pDG780 }\end{array}$ \\
\hline $\begin{array}{l}\text { Kan check } \\
\text { rev }\end{array}$ & CTGCCTCСТCАТССТСТTCАТСC & $\begin{array}{l}\text { Sequencing of kan cassette amplified } \\
\text { from pDG780 }\end{array}$ \\
\hline
\end{tabular}

${ }^{\#}$ restriction sites are underlined, introduced mutations or special sequences are bold, promoters are italic

* LFH-PCR: long flanking homology PCR 


\section{Plasmids}

Table. 12. Plasmids used in this work

\begin{tabular}{|c|c|c|c|}
\hline Plasmid & Resistance & Description & Reference \\
\hline pAC6 & $\begin{array}{l}\text { Ampicillin/ } \\
\text { Chloramphenicol }\end{array}$ & Transcriptional lacZ fusion & Stülke et al., 1997 \\
\hline $\mathrm{pAC7}$ & $\begin{array}{l}\text { Ampicillin/ } \\
\text { Kanamycin }\end{array}$ & Translational lac Z fusion & Weinrauch et al., 1991 \\
\hline $\mathrm{pAG} 2$ & Ampicillin & Overproduction of His-tagged HPr & Galinier et al., 1997 \\
\hline pDG148 & $\begin{array}{l}\text { Ampicillin/ } \\
\text { Kanamycin }\end{array}$ & $\begin{array}{l}\text { Overexpression under the control of } \\
\text { Pspac promoter }\end{array}$ & Joseph et al., 2001 \\
\hline pDG780 & $\begin{array}{l}\text { Amplicillin/ } \\
\text { Kanamycin }\end{array}$ & Amplification of kanamycin cassette & $\begin{array}{l}\text { Guerout-Fleury et al., } \\
1995\end{array}$ \\
\hline pdr79 & $\begin{array}{l}\text { Ampicillin/ } \\
\text { Spectinomycin }\end{array}$ & $\begin{array}{l}\text { Integration of a target protein in } a m y E \\
\text { locus under the control of Pspac promoter }\end{array}$ & Pan et al., 2001 \\
\hline pdr90 & $\begin{array}{l}\text { Ampicillin/ } \\
\text { Spectinomycin }\end{array}$ & $\begin{array}{l}\text { Integration of a target protein in amyE } \\
\text { locus under the control of Phyper-spac } \\
\text { promoter }\end{array}$ & Srivatsan et al., 2008 \\
\hline pdr110 & $\begin{array}{l}\text { Ampicillin/ } \\
\text { Spectinomycin }\end{array}$ & $\begin{array}{l}\text { Integration of a target protein in amyE } \\
\text { locus under the control of Pspank } \\
\text { promoter }\end{array}$ & Rokop et al., 2004 \\
\hline pdr111 & $\begin{array}{l}\text { Ampicillin/ } \\
\text { Spectinomycin }\end{array}$ & $\begin{array}{l}\text { Integration of a target protein in amyE } \\
\text { locus under the control of Phyper-spank } \\
\text { promoter }\end{array}$ & Rokop et al., 2004 \\
\hline pE5635 & Ampicillin & Overproduction of His-tagged PrpC & Gaidenko et al., 2002 \\
\hline pGP205 & Ampicillin & Overproduction of His-tagged HPrK/P & Hanson et al., 2002 \\
\hline pGP211 & Ampicillin & Disruption of $h p r K$ & Hanson et al., 2002 \\
\hline pGP380 & $\begin{array}{l}\text { Ampicillin/ } \\
\text { Erythromycin }\end{array}$ & $\begin{array}{l}\text { Overproduction of N-terminal Strep } \\
\text { tagged target protein }\end{array}$ & Herzberg et al., 2007 \\
\hline pGP811 & $\begin{array}{l}\text { Ampicillin/ } \\
\text { Erythromycin }\end{array}$ & Disruption of $p t s I$ & Schmalisch, 2004 \\
\hline pHCMC05 & $\begin{array}{l}\text { Ampicillin/ } \\
\text { Chloramphenicol }\end{array}$ & $\begin{array}{l}\text { Overexpression under the control of } \\
\text { Pspac promoter }\end{array}$ & Nguyen et al., 2005 \\
\hline pIW11xylR & $\begin{array}{l}\text { Ampicillin/ } \\
\text { Ermthromycin }\end{array}$ & Inactivation of $x y l R$ gene & Kraus et al., 1994 \\
\hline $\begin{array}{l}\text { pMUTIN- } \\
\text { cMyc }\end{array}$ & $\begin{array}{l}\text { Ampicillin/ } \\
\text { Erythromycin }\end{array}$ & $\begin{array}{l}\text { Construction of C-terminally c-Myc } \\
\text { tagged target protein. }\end{array}$ & Kaltwasser et al., 2002 \\
\hline $\begin{array}{l}\text { pMUTIN- } \\
\text { HA }\end{array}$ & $\begin{array}{l}\text { Ampicillin/ } \\
\text { Erythromycin }\end{array}$ & $\begin{array}{l}\text { Construction of C-terminally HA tagged } \\
\text { target protein }\end{array}$ & Kaltwasser et al., 2002 \\
\hline $\begin{array}{l}\text { pMUTIN- } \\
\text { FLAG }\end{array}$ & $\begin{array}{l}\text { Ampicillin/ } \\
\text { Erythromycin }\end{array}$ & $\begin{array}{l}\text { Construction of C-terminally FLAG } \\
\text { tagged target protein }\end{array}$ & Kaltwasser et al., 2002 \\
\hline pSG1729 & $\begin{array}{l}\text { Ampicillin/ } \\
\text { Spectinomycin }\end{array}$ & $\begin{array}{l}\text { Production of N-terminal gfpmutl fusion } \\
\text { with the target protein under control of } \\
\text { Pxyl. Integrates in amyE locus }\end{array}$ & $\begin{array}{l}\text { Lewis and Marston, } \\
1999\end{array}$ \\
\hline
\end{tabular}




\begin{tabular}{|c|c|c|c|}
\hline Plasmid & Resistance & Description & Reference \\
\hline pSG1151 & $\begin{array}{l}\text { Ampicillin/ } \\
\text { Chloramphenicol }\end{array}$ & $\begin{array}{l}\text { Production of } \mathrm{C} \text { terminal gfpmutl fusion } \\
\text { with the target protein. Allows integration } \\
\text { in the native locus }\end{array}$ & $\begin{array}{l}\text { Lewis and Marston, } \\
1999\end{array}$ \\
\hline pSG1187 & $\begin{array}{l}\text { Ampicillin/ } \\
\text { Chloramphenicol }\end{array}$ & $\begin{array}{l}\text { Production of C-terminal } y f p \text { fusion with } \\
\text { the target protein under control. Allows } \\
\text { integration in the native locus and the } \\
\text { expression of the fusion protein driven by } \\
\text { natural promoter }\end{array}$ & Feucht and Lewis, 2001 \\
\hline pSG1164 & $\begin{array}{l}\text { Ampicillin/ } \\
\text { Chloramphenicol }\end{array}$ & $\begin{array}{l}\text { Production of C terminal gfpmutl fusion } \\
\text { with the target protein. Allows integration } \\
\text { in the native locus and the expression of } \\
\text { the downstream genes under the control } \\
\text { of Pxyl. }\end{array}$ & $\begin{array}{l}\text { Lewis and Marston, } \\
1999\end{array}$ \\
\hline pSG1170 & $\begin{array}{l}\text { Ampicillin/ } \\
\text { Chloramphenicol }\end{array}$ & $\begin{array}{l}\text { Production of C terminal gfpmutl fusion } \\
\text { with the target protein. Allows integration } \\
\text { in the native locus and the expression of } \\
\text { the downstream genes under the control } \\
\text { of Pspac promoter }\end{array}$ & $\begin{array}{l}\text { Lewis and Marston, } \\
1999\end{array}$ \\
\hline pSG1154 & $\begin{array}{l}\text { Ampicillin/ } \\
\text { Spectinomycin }\end{array}$ & $\begin{array}{l}\text { Production of C-terminal gfpmut } 1 \text { fusion } \\
\text { with the target protein under control of } \\
\text { Pxyl. Integrates in amyE locus }\end{array}$ & $\begin{array}{l}\text { Lewis and Marston, } \\
1999\end{array}$ \\
\hline
\end{tabular}

Table. 13. Plasmids constructed in this work

\begin{tabular}{|c|c|c|c|}
\hline Plasmid & Construction & Description & Reference \\
\hline pGP633 & pDG148/StuI & PCR-Prod $c r h$ orf, SK1+SK2/StuI & \\
\hline pGP634 & pAC6/EcoRI + SacI & $\begin{array}{l}\text { PCR-Prod } h p r K \text { orf }+700 \mathrm{bp} \text { upstream of } \\
\text { the orf, SK3+SK } 4 / E c o R I+S a c I \text {. }\end{array}$ & \\
\hline pGP636 & $\begin{array}{l}\text { pHCMC05/ } \\
\text { BamHI+XmaI }\end{array}$ & $\begin{array}{l}\text { PCR-Prod yvoE orf, SK15+SK16/ } \\
\text { BamHI+XmaI }\end{array}$ & \\
\hline pGP637 & $\begin{array}{l}\text { pHCMC05/ } \\
\text { BamHI+XmaI }\end{array}$ & $\begin{array}{l}\text { PCR-Prod lgt-yvoF genes, SK17+SK18/ } \\
\text { BamHI+XbaI }\end{array}$ & \\
\hline pGP638 & $\begin{array}{l}\text { pDG148/ } \\
\text { AvaI+BamHI }\end{array}$ & $\begin{array}{l}\text { PCR-Prod xynP }(-279 \text { to }+86) \\
\text { SK27+SK28 / AvaI+BamHI }\end{array}$ & \\
\hline pGP639 & $\begin{array}{l}\text { pDG148/ } \\
\text { AvaI+BamHI }\end{array}$ & $\begin{array}{l}\text { PCR-Prod } x y n P(-279 \text { to }+380) \text {, } \\
\text { SK27+SK29/AvaI }+ \text { BamHI }\end{array}$ & \\
\hline pGP640 & pDG148/StuI & PCR-Prod $c c p A$ orf, SK30+SK31/StuI & \\
\hline pGP641 & $\begin{array}{l}\text { pGP380/ } \\
\text { BamHI+PstI }\end{array}$ & $\begin{array}{l}\text { PCR-Prod crh orf, SK32+SK33/ } \\
\text { BamHI+PstI }\end{array}$ & \\
\hline pGP642 & $\begin{array}{l}\text { pGP380/ } \\
\text { BamHI+HindIII }\end{array}$ & $\begin{array}{l}\text { PCR-Prod hprK orf, SK11+KS12/ } \\
\text { BamHI+HindIII }\end{array}$ & \\
\hline
\end{tabular}




\begin{tabular}{|c|c|c|c|}
\hline Plasmid & Construction & Description & Reference \\
\hline pGP643 & $\begin{array}{l}\text { pGP380/ } \\
\text { BamHI+SalI }\end{array}$ & $\begin{array}{l}\text { PCR-Prod ccpA orf, SK34+SK35/ } \\
\text { BamHI+SalI }\end{array}$ & \\
\hline pGP644 & pDG148/StuI & PCR-Prod hprK orf, SK42+SK43/ StuI & \\
\hline pGP645 & pDG148/StuI & $\begin{array}{l}\text { PCR-Prod } h p r K \text { G158A, SK42+SK43/ } \\
\text { StuI, pGP } 407 \text { as a template to amplify the } \\
\text { PCR product }\end{array}$ & \\
\hline pGP646 & $\begin{array}{l}\text { pdr79/ } \\
\text { HindIII+SalI }\end{array}$ & $\begin{array}{l}\text { PCR-Prod ptsH orf, SK } 46+\text { SK } 47 / \\
\text { HindIII+SalI, linearization of the plasmid } \\
\text { via } S c a I\end{array}$ & \\
\hline pGP647 & $\begin{array}{l}\text { pdr79/ } \\
\text { HindIII+SalI }\end{array}$ & $\begin{array}{l}\text { PCR-Prod } c r h \text { orf, SK57+SK58/ } \\
\text { HindIII+SalI, linearization of the plasmid } \\
\text { via } S c a \mathrm{I}\end{array}$ & \\
\hline pGP648 & $\begin{array}{l}\mathrm{pSG} 1729 / \\
\text { BamHI+EcoRI }\end{array}$ & $\begin{array}{l}\text { PCR-Prod } y v c J \text { orf, SK48+SK49/ } \\
\text { BamHI+EcoRI, linearization of the } \\
\text { plasmid via } S c a \text { I }\end{array}$ & \\
\hline pGP649 & $\begin{array}{l}\text { pSG1729/ } \\
\text { BamHI+EcoRI }\end{array}$ & $\begin{array}{l}\text { PCR-Prod } y v c K \text { orf, SK50+SK51/ } \\
\text { BamHI+EcoRI, linearization of the } \\
\text { plasmid via } S c a \text { I }\end{array}$ & \\
\hline pGP650 & $\begin{array}{l}\text { pGP380/ } \\
\text { BamHI+HindIII }\end{array}$ & $\begin{array}{l}\text { PCR-Prod hprKG158A, SK11+KS12/ } \\
\text { BamHI+HindIII, pGP407 used as a } \\
\text { template for the amplification of the insert }\end{array}$ & Singh et al., 2008 \\
\hline
\end{tabular}

\section{Strains}

Table. 14. Strains used in this work

\begin{tabular}{|c|c|c|}
\hline Strain & Genotype & Reference \\
\hline \multicolumn{3}{|c|}{ Escherichia coli } \\
\hline $\begin{array}{l}\text { BL21 } \\
\text { (DE3) }\end{array}$ & $\begin{array}{l}\text { F- lon ompT rBmB hsdS gal (cIts857ind1 Sam7 nin5 } \\
\text { lacUV5- T7 gene1) }\end{array}$ & Sambrook et al., 1989 \\
\hline DH5 $\alpha$ & 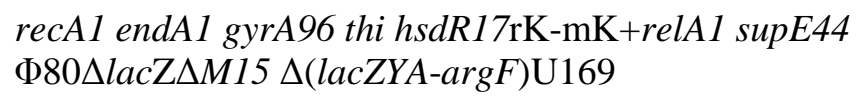 & Sambrook et al., 1989 \\
\hline NM522 & $\begin{array}{l}\text { supE thi-1 } \Delta(\text { lac-proAB }) \Delta(\text { mcrB-hsdSM }) 5\left(\mathrm{r}_{\mathrm{K}}-\mathrm{m}_{\mathrm{K}}\right)\left[\mathrm{F}^{6}\right. \\
\left.\text { proAB lacl }{ }^{\mathrm{q}} \mathrm{Z} \Delta \mathrm{M} 15\right]\end{array}$ & Gough and Murray, 1983 \\
\hline \multicolumn{3}{|c|}{ Bacillus subtilis } \\
\hline 168 & $\operatorname{trp} C 2$ & Laboratory collection \\
\hline GP202 & $\operatorname{trpC} 2$ hprK::spc & Hanson et al., 2002 \\
\hline GP505 & $\operatorname{trpC} 2$ ptsH-H15A amyE::(lacZ cat) & Schmalisch, 2004 \\
\hline GS4 & $\operatorname{trpC2} y v c J(+369):: p X 2(c a t)$ & Boris Görke; unpublished \\
\hline GS5 & $\operatorname{trpC} 2 y v c K(+367):: p X 2(c a t)$ & Boris Görke; unpublished \\
\hline MZ303 & $\operatorname{trpC} 2 \Delta p t s H:: c a t$ & Arnaud et al., 1996 \\
\hline
\end{tabular}




\begin{tabular}{|c|c|c|}
\hline Strain & Genotype & Reference \\
\hline OMG401 & $\operatorname{trp} C 2 \Delta \operatorname{prpC}:: a p h A 3$ & Madec et al., 2002 \\
\hline OMG302 & $\operatorname{trp} C 2 \Delta p r k C:: a p h A 3$ & Madec et al., 2002 \\
\hline PB703 & $\operatorname{trp} C 2 \Delta p r p C$ amyE::(ctc-lacZ cat) & Gaidenko et al., 2002 \\
\hline QB5223 & $\operatorname{trpC2} \mathrm{ptsH1}$ & $\begin{array}{l}\text { Martin-Verstraete } \text { et al., } \\
1995\end{array}$ \\
\hline QB5407 & $\operatorname{trp} C 2$ сcpA::Tn917 spc & Faires et al., 1999 \\
\hline QB7096 & $\operatorname{trp} C 2$ crh::aphA3 & Presecan-Siedel et al., 1999 \\
\hline QB7097 & $\operatorname{trpC2} \operatorname{crh::spc}$ & $\begin{array}{l}\text { Martin-Verstraete; } \\
\text { unpublished }\end{array}$ \\
\hline QB7101 & $\operatorname{trpC} 2$ ptsH1 crh::spc & $\begin{array}{l}\text { Martin-Verstraete; } \\
\text { unpublished }\end{array}$ \\
\hline QB7102 & $\operatorname{trpC} 2$ ptsH1 crh::aphA3 & Presecan-Siedel et al., 1999 \\
\hline QB7108 & $\operatorname{trpC2}$ ptsH1 crh::aphA3, amyE:(Pspac crh cat) & Galinier et al., 1997 \\
\hline QB7144 & $\operatorname{trpC} 2$ amyE::xynP-lacZ & Galinier et al., 1999 \\
\hline QB7160 & $\operatorname{trp} C 2 \Delta h p r K:: a p h A 3$ amyE::( $\mathrm{P} \Delta \mathrm{B}$ levD-lacZ $\left.\mathrm{Cm}^{\mathrm{S}}\right)$ & $\begin{array}{l}\text { Martin-Verstraete } \text { et al., } \\
1999\end{array}$ \\
\hline
\end{tabular}

Table. 15. Strains constructed in this work

\begin{tabular}{|c|c|c|c|}
\hline Strain & Genotype & Construction $^{\mathrm{a}}$ & Reference \\
\hline GP270 & trpC2 xylR::ermC & $\begin{array}{l}\text { pIW11xylR } \rightarrow \\
\text { WT168 }\end{array}$ & Singh et al., 2008 \\
\hline GP271 & $\operatorname{trp} C 2$ xylR::ermC crh::aphA3 & QB7096 $\rightarrow$ GP270 & \\
\hline GP272 & $\begin{array}{l}\operatorname{trp} C 2 \Delta p r p C \text { amyE::(ctc-lacZ } \\
\text { cat) hprK::spc }\end{array}$ & pGP211 $\rightarrow$ PB703 & \\
\hline GP273 & $\begin{array}{l}\operatorname{trp} C 2 \triangle \operatorname{prp} C \text { amyE::(pAC7 } \\
\text { aphA3) }\end{array}$ & $\mathrm{pAC7} \rightarrow \mathrm{PB} 703$ & \\
\hline GP274 & $\begin{array}{l}\operatorname{trp} C 2 \Delta p r p C \text { amyE::(xynP-lacZ } \\
\text { cat) }\end{array}$ & QB7144 $\rightarrow$ GP273 & \\
\hline GP275 & $\begin{array}{l}\text { trpC2 } \Delta p r p C \text { amyE::(xynP-lacZ } \\
\text { cat) xylR::ermC }\end{array}$ & $\begin{array}{l}\text { pIW11xylR } \rightarrow \\
\text { GP274 }\end{array}$ & \\
\hline GP276 & $\begin{array}{l}\operatorname{trp} C 2 \Delta \text { prpC amyE::(xynP-lacZ } \\
\text { cat) xylR::ermC crh::aphA3 }\end{array}$ & QB7096 $\rightarrow$ GP275 & \\
\hline GP277 & $\begin{array}{l}\operatorname{trpC} 2 \Delta p r p C \text { amyE::(xynP-lacZ } \\
\text { cat) xylR::ermC hprK::spc }\end{array}$ & pGP211 $\rightarrow$ GP275 & \\
\hline GP278 & $\begin{array}{l}\operatorname{trp} C 2 \text { amyE::(xynP-lacZ cat) } \\
\text { xylR::ermC }\end{array}$ & $\begin{array}{l}\text { pIW11xylR } \rightarrow \\
\text { QB7144 }\end{array}$ & Singh et al., 2007 \\
\hline GP279 & $\begin{array}{l}\operatorname{trp} C 2 \text { amyE::(xynP-lacZ cat) } \\
\text { xylR::ermC crh::aphA3 }\end{array}$ & QB7096 $\rightarrow$ GP278 & Singh et al., 2008 \\
\hline
\end{tabular}




\begin{tabular}{|c|c|c|c|}
\hline Strain & Genotype & Construction $^{\text {a }}$ & Reference \\
\hline GP280 & $\begin{array}{l}\operatorname{trpC} 2 \text { amyE::(xynP-lacZ cat) } \\
\text { xylR::ermC hprK::spc }\end{array}$ & pGP211 $\rightarrow$ GP278 & \\
\hline GP281 & $\begin{array}{l}\operatorname{trp} C 2 \text { amyE::(xynP-lacZ cat) } \\
\text { xylR::ermC } \triangle \text { prpC }:: a p h A 3\end{array}$ & $\begin{array}{l}\text { OMG } 401 \rightarrow \\
\text { GP278 }\end{array}$ & Singh et al., 2007 \\
\hline GP282 & $\begin{array}{l}\text { trpC2 amyE::(xynP-lacZ cat) } \\
\text { xylR::ermC } \triangle \text { prkC::aphA3 }\end{array}$ & $\begin{array}{l}\text { OMG } 302 \rightarrow \\
\text { GP278 }\end{array}$ & \\
\hline GP283 & $\begin{array}{l}\text { trpC2 } 2 \text { hrK ::spc amyE::(hprK } \\
\text { cat) }\end{array}$ & pGP634 $\rightarrow$ GP202 & \\
\hline GP284 & trpC2 ptsH1 xylR::ermC & $\begin{array}{l}\text { pIW11xylR } \rightarrow \\
\text { QB5223 }\end{array}$ & Singh et al., 2008 \\
\hline GP285 & $\begin{array}{l}\operatorname{trpC} 2 \text { amyE::(xynP-lacZ cat) } \\
\text { xylR::ermC } \Delta p r p C:: a p h A 3 \\
\text { hprK::spc }\end{array}$ & pGP211 $\rightarrow$ GP281 & \\
\hline GP286 & $\begin{array}{l}\operatorname{trpC} 2 \text { amyE::(xynP-lacZ cat) } \\
\text { xylR::ermC } \Delta p r k C:: \text { aphA3 } \\
\text { hprK::spc }\end{array}$ & pGP211 $\rightarrow$ GP282 & \\
\hline GP287 & $\operatorname{trpC} 2$ ptsH1 crh::spc xylR::ermC & $\begin{array}{l}\text { pIW11xylR } \rightarrow \\
\text { GP7101 }\end{array}$ & Singh et al., 2008 \\
\hline GP288 & $\begin{array}{l}\text { trpC2 hprK::spc amyE::(PhprK } \\
\text { hprK cat) xylR::ermC }\end{array}$ & $\begin{array}{l}\text { pIW11xylR } \rightarrow \\
\text { GP283 }\end{array}$ & \\
\hline GP289 & $\operatorname{trpC} 2$ xylR::ermC $\Delta$ hprK::aphA3 & QB7160 $\rightarrow$ GP270 & Singh et al., 2008 \\
\hline GP290 & $\begin{array}{l}\operatorname{trpC} 2 \text { xylR::ermC } \Delta \text { hprK::aphA3 } \\
\text { amyE::(PhprK hprK cat) }\end{array}$ & pGP634 $\rightarrow$ GP289 & \\
\hline GP291 & $\begin{array}{l}\text { trpC2 } p t s H 1 \text { crh::aphA3 } \\
\text { amyE::(Pspac-ptsH spc) }\end{array}$ & pGP646 $\rightarrow$ GP293 & \\
\hline GP292 & $\begin{array}{l}\operatorname{trp} C \text { xylR::erm } C \text { amyE::(xynP- } \\
\text { lacZ cat) crh::spc }\end{array}$ & QB7097 $\rightarrow$ GP278 & \\
\hline GP293 & $\begin{array}{l}\text { trpC2 ptsH1 crh::aphA3, } \\
\text { xylR::ermC }\end{array}$ & $\begin{array}{l}\text { pIW11xylR } \rightarrow \\
\text { QB7102 }\end{array}$ & \\
\hline GP294 & $\begin{array}{l}\text { trpC2 xylR::ermC ptsHI } \\
\text { crh::phA3 amyE::( Pspac -crh } \\
\text { spc) }\end{array}$ & pGP647 $\rightarrow$ GP293 & \\
\hline GP295 & $\begin{array}{l}\operatorname{trpC} 2 \text { ptsH1 crh::aphA3 } \\
\text { amyE::(Pspac-crh } s p c)\end{array}$ & pGP647 $\rightarrow$ QB7102 & \\
\hline GP296 & $\begin{array}{l}\operatorname{trpC} 2 \Delta p t s H:: c a t \text { amyE::(Pspac - } \\
\text { ptsH spc) }\end{array}$ & pGP646 $\rightarrow$ MZ303 & \\
\hline GP297 & $\operatorname{trp} C 2$ xylR::ermC crh::spc & QB7097 $\rightarrow$ GP270 & Singh et al., 2008 \\
\hline GP298 & $\begin{array}{l}\operatorname{trp} C 2 \text { xylR::ermC amyE::(lacZ } \\
\text { cat) }\end{array}$ & pAC6 $\rightarrow$ GP270 & \\
\hline GP299 & $\begin{array}{l}\operatorname{trpC} 2 \text { xylR::ermC ptsH-H15A } \\
\text { amyE::(lacZ cat) }\end{array}$ & $\begin{array}{l}\text { pIWIIxylR } \rightarrow \\
\text { GP505 }\end{array}$ & \\
\hline GP851 & $\begin{array}{l}\operatorname{trpC} 2 \text { xylR::ermC } \Delta l g t- \\
\text { yvoF::aphA3 }\end{array}$ & $\begin{array}{l}\text { LFH PCR product } \\
\rightarrow \mathrm{GP} 270\end{array}$ & \\
\hline
\end{tabular}




\begin{tabular}{|c|c|c|c|}
\hline Strain & Genotype & Construction $^{\mathrm{a}}$ & Reference \\
\hline GP852 & $\operatorname{trp} C 2$ xylR::ermC $\Delta y v o E:: a p h A 3$ & $\begin{array}{l}\text { LFH PCR product } \\
\rightarrow \text { GP } 270\end{array}$ & \\
\hline GP853 & $\begin{array}{l}\operatorname{trp} C 2 \text { xylR::erm } C \text { ccpA::Tn917 } \\
\text { spc }\end{array}$ & QB5407 $\rightarrow$ GP270 & Singh et al., 2008 \\
\hline GP854 & $\begin{array}{l}\operatorname{trpC} 2 \text { yvcK }(+367):: p X 2 \text { (cat) } \\
\text { amyE::(Pxyl-GFP spc) }\end{array}$ & pSG1729 $\rightarrow$ GS5 & \\
\hline GP855 & $\begin{array}{l}\text { trpC2 hprK::spc amyE::(hrpK } \\
\text { cat) xylR::ermC } \Delta l g t- \\
\text { yvoF::aphA3 }\end{array}$ & GP851 $\rightarrow$ GP 288 & \\
\hline GP856 & $\operatorname{trpC} 2$ hprK::spc xylR::ermC & pGP211 $\rightarrow$ GP270 & \\
\hline GP857 & $\begin{array}{l}\text { trpC2 hprK::spc xylR::ermC } \Delta \text { lgt- } \\
\text { yvoF::aphA3 }\end{array}$ & GP $851 \rightarrow$ GP 856 & \\
\hline GP858 & $\operatorname{trpC2} \Delta h p r K:: a p h A 3$ & $\mathrm{QB} 7160 \rightarrow \mathrm{Wt} 168$ & Singh et al., 2008 \\
\hline GP859 & $\begin{array}{l}\operatorname{trp} C 2 \text { xylR::ermC } \triangle y v o E- \\
\text { yvoF::aphA3 }\end{array}$ & $\begin{array}{l}\text { LFH PCR product } \\
\rightarrow \mathrm{GP} 270\end{array}$ & \\
\hline GP860 & $\operatorname{trpC} 2$ crh::aphA3 & $\begin{array}{l}\text { QB7096 } \rightarrow \text { Wt168 } \\
\text { "Genotype identical } \\
\text { to QB7096" }\end{array}$ & \\
\hline GP861 & $\begin{array}{l}\operatorname{trp} C 2 \text { xylR::ermC } \triangle y v o D- \\
\text { yvoF::aphA3 }\end{array}$ & $\begin{array}{l}\text { LFH PCR product } \\
\rightarrow \mathrm{GP} 270\end{array}$ & \\
\hline GP862 & $\operatorname{trpC} 2$ amyE::(Pspac-ptsH spc) & pGP646 $\rightarrow$ Wt 168 & \\
\hline GP863 & $\begin{array}{l}\text { trpC2 xylR::ermC amyE::(Pspac- } \\
\text { ptsH spc) }\end{array}$ & pGP646 $\rightarrow$ GP270 & \\
\hline GP864 & $\operatorname{trp} C 2 \Delta p t s I:: e r m C$ & pGP811 $\rightarrow$ Wt168 & Singh et al., 2008 \\
\hline GP865 & trpC2 crh::aphA3 $\Delta p t s I:: e r m C$ & pGP811 $\rightarrow$ QB7096 & \\
\hline GP866 & $\begin{array}{l}\operatorname{trpC2}, y v c J(+369):: p X 2(c a t) \\
\text { amyE::(Pxyl-yvcJ-GFP spc) }\end{array}$ & pGP648 $\rightarrow$ GS4 & \\
\hline GP867 & $\begin{array}{l}\operatorname{trpC} 2 \text { yvcK }(+367):: p X 2(c a t) \\
\text { amyE::(Pxyl.-yvcK-GFP spc) }\end{array}$ & pGP649 $\rightarrow$ GS5 & \\
\hline
\end{tabular}

\footnotetext{
${ }^{a}$ Arrows indicate construction by transformation.
} 


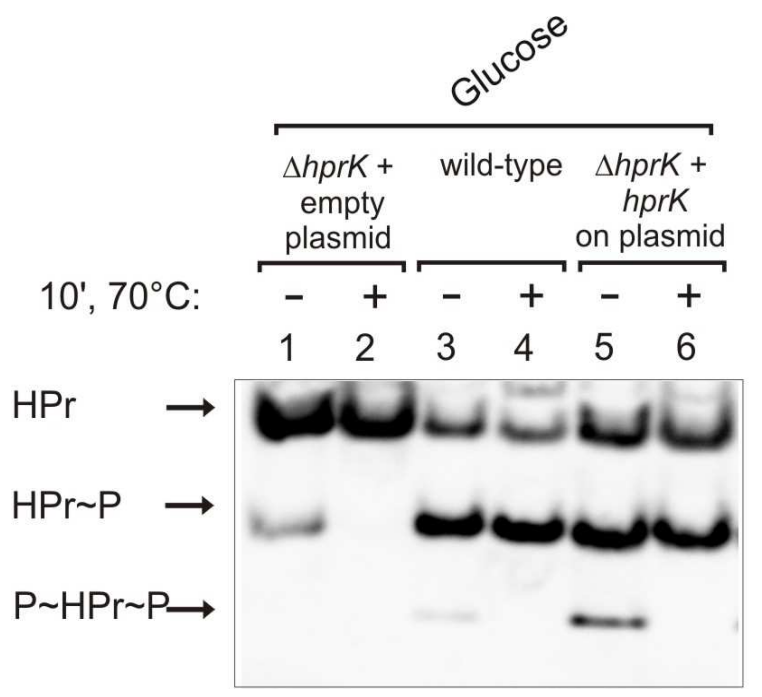

Figure. 23. The level of unphosphorylated HPr and $\operatorname{HPr}(\operatorname{Ser}-\mathrm{P})$ in a strain expressing hprk from a multicopy vector (pGP642) is similar to the wild type strain. Cells were grown in CSE + glucose and harvested at logarithmic phase as described before (Singh et al., 2007). $2 \mu \mathrm{g}$ of total cell protein, each was separated on a $12 \%$ native PAA gel and HPr was detected by immuno-blotting in a western blot analysis. To discriminate between $\operatorname{HPr}(\mathrm{Ser}-\mathrm{P})$ and $\mathrm{HPr}(\mathrm{His}-\mathrm{P})$, an aliquot of each cell extract was heated at $70{ }^{\circ} \mathrm{C}$ prior to loading (even numbered lanes). Histidine phosphorylation is heat labile and thus the two singly phosphorylated forms of HPr can be easily distinguished. In $\Delta h p r K$ strain with empty plasmid (pGP380), no $\mathrm{HPr}(\operatorname{Ser}-\mathrm{P})$ is detected (lane 1 and 2). Introducing the hprK on a multicopy plasmid (pGP642) restored the "wild type" phosphorylation pattern of HPr (compare lane3, 4 and lane 5,6).

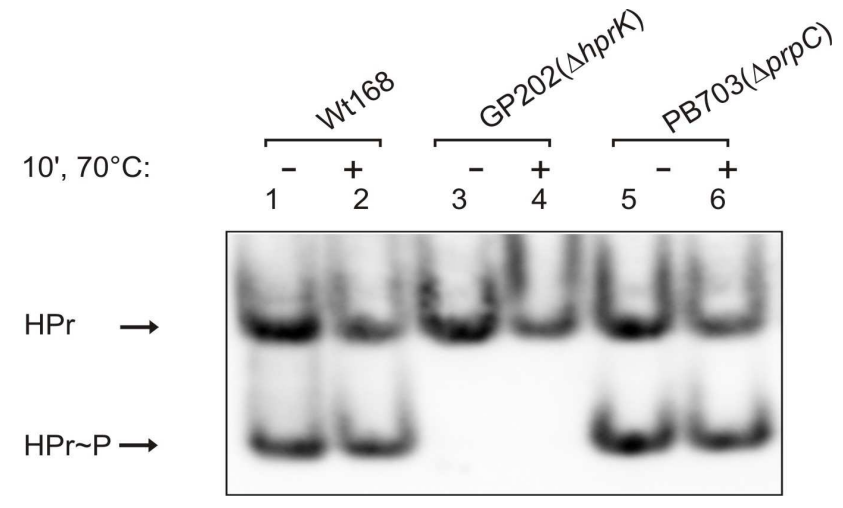

Figure. 24. Determination of the phosphorylation state of HPr in $\triangle p r p C$ and $\Delta h p r K$ mutants. Cells were grown in CSE + glucose and harvested at logarithmic phase as described before (Singh $e t$ al., 2007). $1 \mu \mathrm{g}$ of total cell protein, each was separated on a $10 \%$ native PAA gel and HPr was detected by immuno-blotting in a western blot analysis. In $\Delta h p r K$ strain, none of the phosphorylated form of HPr (Ser-P or His-15) was detected. In wt strain, 48\% of the HPr was phosphorylated at serine residue, rest being the unphosphorylated form. Similar phosphorylation pattern of HPr was detected in PB703 ( $\triangle p r p C)$ strain, where $52 \%$ of the $\operatorname{HPr}(\operatorname{Ser}-\mathrm{P})$ was detected. 
Table. 16. Catabolite repression of $x y n P-l a c Z$ fusion in the presence of glucose in $\triangle p r p C$ mutant. The values are the average of at least two independent experiments. Standard deviations are shown in parentheses.

\begin{tabular}{cccc}
\hline \multicolumn{3}{c}{$\beta$-galactosidase activity in units/mg of protein } \\
\hline Carbon & GP278 & GP280 & GP275 \\
source $^{\mathrm{a}}$ & $\Delta x y l R$, & $\Delta x y l R, \Delta h p r K$, & $\Delta x y l R$, \\
& amyE::xynP-lacZ & amyE::xynP-lacZ & $\Delta p r p C$, amyE::xynP- \\
& & lacZ \\
\hline- & $740(41)$ & $1110(282)$ & $498(175)$ \\
Glucose & $31(3)$ & $1941(674)$ & $20(2)$ \\
\hline
\end{tabular}

added to CSE medium $(0.5 \%)$

\section{Intracellular Glucose-6-P (G6P) concentration}

Protein-free cell extracts for the G6P concentrations in B. subtilis were prepared as described earlier with few modifications (Mijakovic et al., 2002). Briefly, cells of the B. subtilis wild type strain 168 were grown in $50 \mathrm{ml}$ of CSE medium in the presence of the indicated carbon sources $(0.5 \%, w / v)$. For each growth condition, at least three independent experiments were carried out. Cultures were harvested by centrifugation at room temperature for 5 minutes at $10,000 \mathrm{~g}$ followed by freezing the pellet in liquid nitrogen. Pellets were resuspended in $0.6 \mathrm{M}$ of cold perchloric acid and subsequently kept on ice for 20 minutes. The precipitated proteins and cell debris were removed by centrifugation $\left(4^{\circ} \mathrm{C}, 5\right.$ minutes, 13,000 rpm). The $\mathrm{pH}$ in the supernatant was subsequently adjusted to 7.4 with a solution of cold $0.6 \mathrm{M} \mathrm{KOH}$ in $0.5 \mathrm{M}$ glycyl glycine buffer ( $\mathrm{pH}$ 7.4). The precipitated $\mathrm{KClO}_{4}$ was removed by centrifugation. Aliquots of the final supernatants were used to measure the amounts of G6P in a direct photometric assay. For intracellular G6P estimation, reaction mixtures of $1 \mathrm{ml}$ were prepared, which contained $600 \mu \mathrm{l}$ of cell extract and $100 \mathrm{mM}$ of glycyl-glycine buffer ( $\mathrm{pH} 7.4), 0.3 \mathrm{mM}$ of NADP, $5 \mathrm{mM}$ of $\mathrm{MgCl}_{2}$ and 3.3 units of Glucose-6-phosphate dehydrogenase. The increase in absorbance at $340 \mathrm{~nm}$ was measured as indicator of the NADPH formation. 
Table. 17. Intracellular amounts of G6P in wild type 168 grown in the presence of different carbon sources. The G6P concentrations were calculated by correlating the obtained values to a standard calibration curve (data not shown).

\begin{tabular}{lc}
\hline Carbon source $^{\mathrm{a}}$ & G6P $(\mathrm{mM}) *$ \\
\hline Glucose & $0.88(0.09)$ \\
Salicin & $0.34 \quad(0.01)$ \\
Mannitol & - \\
Fructose & $0.43 \quad(0.10)$ \\
Sorbitol & - \\
Glycerol & $0.27 \quad(0.10)$ \\
Gluconate & $0.21 \quad(0.01)$ \\
Arabinose & $0.36(0.13)$ \\
Maltose & $0.65 \quad(0.06)$ \\
Ribose & - \\
CSE & - \\
\hline added to CSE medium $(0.5 \%),-=$ Below detection limit, $*=$ The standard deviations are \\
given in parentheses.
\end{tabular}




\section{Curriculum vitae}

$\begin{array}{ll}\text { Name } & \text { Kalpana Singh } \\ \text { Date of Birth } & \text { 24 May 1982 } \\ \text { Place of Birth } & \text { Haryana, India } \\ \text { Nationality } & \text { Indian }\end{array}$

\section{Education}

1988-1998

High School, D.A.V. Public School, Haryana, India

$1998-2000$

Senior Secondary School, M.V.N. Public School, Haryana, India

2000-2004

Bachelor of Biotechnology, Guru Gobind Singh Indraprastha University, Delhi, India

Thesis: Characterization of the subunit 4 of the RNA polymerase in Saccharomyces cerevisiae.

2004-Present Program in Molecular Biology, International Max-Planck Research School,

Göttingen, Germany

$\mathrm{PhD}$ thesis at the Department of General Microbiology, Institute for Microbiology and Genetics (Supervised by Prof. Jörg Stülke and Dr. Boris Görke)

Title: The role of protein phosphorylation in regulation of carbon catabolite repression in Bacillus subtilis 